\title{
Pattern Formation and Oscillatory Dynamics in a Two-Dimensional Coupled Bulk-Surface Reaction-Diffusion System
}

\author{
Frédéric Paquin-Lefebvre $^{*}$, Wayne Nagata ${ }^{\dagger}$, Michael J. Ward ${ }^{\ddagger}$
}

August 11, 2020

\begin{abstract}
On a two-dimensional circular domain, we analyze the formation of spatio-temporal patterns for a class of coupled bulk-surface reaction-diffusion models for which a passive diffusion process occurring in the interior bulk domain is linearly coupled to a nonlinear reaction-diffusion process on the domain boundary. For this coupled PDE system we construct a radially symmetric steady state solution and from a linearized stability analysis formulate criteria for which this base state can undergo either a Hopf bifurcation, a symmetry-breaking pitchfork (or Turing) bifurcation, or a codimension-two pitchfork-Hopf bifurcation. For each of these three types of bifurcations, a multiple time-scale asymptotic analysis is used to derive normal form amplitude equations characterizing the local branching behavior of spatio-temporal patterns in the weakly nonlinear regime. Among the novel aspects of this weakly nonlinear analysis are the two-dimensionality of the bulk domain, the systematic treatment of arbitrary reaction kinetics restricted to the boundary, the bifurcation parameters which arise in the boundary conditions, and the underlying spectral problem where both the differential operator and the boundary conditions involve the eigenvalue parameter. The normal form theory is illustrated for both Schnakenberg and Brusselator reaction kinetics, and the weakly nonlinear results are favorably compared with numerical bifurcation results and results from time-dependent PDE simulations of the coupled bulk-surface system. Overall, the results show the existence of either subcritical or supercritical Hopf and symmetry-breaking bifurcations, and mixed-mode oscillations characteristic of codimension-two bifurcations. Finally, the formation of global structures such as large amplitude rotating waves is briefly explored through PDE numerical simulations.
\end{abstract}

\section{Introduction}

If a passive linear diffusion process in a bounded domain is coupled to a nonlinear reaction-diffusion process on the domain boundary, spatio-temporal patterns can occur that otherwise would not be present without this bulk-surface coupling. Such a pattern formation mechanism is relevant in a variety of applications in which boundaries play an active role in the overall dynamics. For instance, in some biological cell signalling contexts certain proteins cycle from an active cellular membrane to a cytoplasmic bulk via adsorption and desorption processes. Applications of this type include the formation of surface-bound Turing patterns through symmetry-breaking instabilities (cf. [18], [22], [23]) as well as the onset of Min-protein pole-to-pole oscillations prior to cell division in E. Coli (cf. [14], [15]). In many prior studies (eg. [22], [23], [18]), the coupled bulk-surface systems have mainly been analyzed through either a linear stability analysis, which typically involves finding the conditions for a Turing-type diffusion-driven instability, or from time-dependent PDE numerical simulations (cf. [20], [19]).

In a simplified geometry consisting of a 1-D spatial bulk domain, these models become coupled PDE-ODE models, and were studied in [7], [9], [8] and [10]. There, dynamically active units, modeled by nonlinear ODEs, are spatially segregated and are coupled through a linear bulk diffusion field. This setup serves as a modeling paradigm for the study of synchrony under diffusion sensing. In contrast to the classical types of PDE-ODE models where the coupling occurs in all of space (cf. [16]), the type of coupling considered here, and in [7]-[10], is restricted to the boundaries, and is expressed in terms of Robin-type boundary conditions. In [7]-[10], this class of 1-D coupled PDE-ODE systems was analyzed through a combination of linear stability analysis, direct numerical PDE simulations, and numerical bifurcation software. The numerical bifurcation studies have allowed for the computation of global branches of synchronous and asynchronous periodic solutions in terms of bulk diffusion coefficients and coupling rates. As an extension of the linear stability theory, in [9] a weakly nonlinear analysis was developed to study the local branching behavior of synchronous oscillations for the idealized case of a single bulk species diffusing between two identical membranes, each consisting of a single active species.

To extend this previous work, our goal in this paper is to provide a comprehensive weakly nonlinear, or normal form, analysis, to study the various bifurcations associated with a class of dimensionless coupled bulk-surface reaction-diffusion systems for which the bulk domain $\Omega$ consists of the disk

$$
\Omega=\left\{x \in \mathbb{R}^{2} \mid\|x\|<R\right\},
$$

\footnotetext{
*Dept. of Mathematics, UBC, Vancouver, Canada. (corresponding author paquinl@math.ubc.ca)

${ }^{\dagger}$ Dept. of Mathematics, UBC, Vancouver, Canada. nagata@math.ubc.ca

${ }^{\ddagger}$ Dept. of Mathematics, UBC, Vancouver, Canada. ward@math.ubc.ca
} 
of radius $R$. In the bulk domain we assume that two bulk species $U, V$ undergo passive diffusion with linear decay in $\Omega$. This leads to the following PDEs in the bulk region

$$
\frac{\partial U}{\partial t}=D_{u} \Delta U-\sigma_{u} U, \quad \frac{\partial V}{\partial t}=D_{v} \Delta V-\sigma_{v} V, \quad x \in \Omega, \quad t>0 .
$$

Here $D_{u}, D_{v}$ are the constant bulk diffusion coefficients, while $\sigma_{u}, \sigma_{v}$ are the constant bulk decay rates. Since $\Omega$ is the disk, the Laplacian $\Delta$ in (1.2) is conveniently written in terms of polar coordinates $(r, \theta)$ as $\Delta=\partial_{r r}+r^{-1} \partial_{r}+r^{-2} \partial_{\theta \theta}$. Next, we assume that the flux normal to the boundary is proportional to the difference between the surface-bound species densities, denoted by $u, v$, and the bulk species densities evaluated on the boundary. This yields linear Robin-type boundary conditions for (1.2):

$$
\left.D_{u} \frac{\partial U}{\partial r}\right|_{r=R}=K_{u}\left(u-\left.U\right|_{r=R}\right),\left.\quad D_{v} \frac{\partial V}{\partial r}\right|_{r=R}=K_{v}\left(v-\left.V\right|_{r=R}\right),
$$

where $K_{u}, K_{v}$ are coupling rate constants, also known as Langmuir rate constants. Finally, on the domain boundary the dynamics of the two surface-bound species are assumed to be governed by a system of reaction-diffusion equations with periodic boundary conditions in the azimuthal coordinate:

$$
\frac{\partial u}{\partial t}=\frac{d_{u}}{R^{2}} \frac{\partial^{2} u}{\partial \theta^{2}}-K_{u}\left(u-\left.U\right|_{r=R}\right)+f(u, v), \quad \frac{\partial v}{\partial t}=\frac{d_{v}}{R^{2}} \frac{\partial^{2} v}{\partial \theta^{2}}-K_{v}\left(v-\left.V\right|_{r=R}\right)+g(u, v) .
$$

Here $f(u, v)$ and $g(u, v)$ are the given reaction kinetics, while $d_{u}, d_{v}$ are surface diffusion coefficients. In the absence of surface diffusion, this model reduces to the coupled PDE-ODE system studied through linear stability analysis in [18] for a slightly different boundary condition and with Gierer-Meinhardt kinetics on the circular membrane. As a remark, since a biologically-realistic membrane possesses some width, the coupled bulk-surface model, defined by (1.2)-(1.4), provides only an approximation to this more complicated setting in the case where the width of the membrane is small in comparison with the characteristic length scale of the bulk domain. Our coupled bulk-surface model, as introduced above, is given in dimensionless form. We refer the reader to Appendix $\S \mathrm{B}$ for a derivation of the system (1.2)-(1.4) from a model with physical units.

Our primary goal herein is to characterize the dynamics of the coupled system in the weakly nonlinear regime near one of three distinct bifurcations; a Hopf bifurcation, for which the spatial mode is trivial with a nonzero temporal frequency, a pitchfork bifurcation, for which the spatial mode is nontrivial with a zero temporal frequency, and finally a codimension-two pitchfork-Hopf bifurcation, which occurs when the previous two bifurcations coincide. By deriving amplitude, or rather, normal form equations, we will characterize the branching behavior in the vicinity of these three bifurcations. Although the use of weakly nonlinear analysis to study pattern formation in reaction-diffusion systems, convection processes, and fluid flows is well-established and ubiquitous in the literature (cf. [3], [26]), the development of a weakly nonlinear theory to characterize pattern formation near bifurcation points of coupled bulk-surface models requires a careful analysis of the spectral problem for the linearization, where both the differential operator and the boundary conditions involve the eigenvalue parameter. This analysis is at the core of performing a weakly nonlinear analysis using a multiple time-scale expansion method. We believe such a spectral problem has not been considered previously in the context of two-dimensional pattern formation problems. We also give a systematic treatment of the three distinct bifurcations for arbitrary reaction kinetics on the surface.

In our formulation, we will suppose that when uncoupled from the bulk domain, the reaction-diffusion system on the surface (1.4) possesses a unique spatially uniform steady state, which is linearly stable with respect to any spatial perturbation. Consequently, we will restrict the parameter space to cases where $d_{u}=d_{v}$ in order to avoid the short-range activation combined with long-range inhibition paradigm, typical of Turing instabilities. Rather than using the surface diffusion coefficients as bifurcation parameters, we will vary the bulk diffusion coefficients and the coupling rates, so that the loss of stability of the base state results from the diffusive coupling with the bulk domain. In terms of these bifurcation parameters that are associated with the boundary conditions (1.3), in $\S 2$ we find that multi-scale expansion methods are particularly convenient for deriving amplitude equations characterizing the local branching behavior.

Although arbitrary reaction kinetics are employed in our analysis, we will apply our weakly nonlinear theory to either the well-known Schnakenberg or Brusselator kinetics. In nondimensional forms, the Schnakenberg kinetics are

$$
f(u, v)=a-u+u^{2} v, \quad g(u, v)=b-u^{2} v ; \quad a, b>0, \quad b-a<(b+a)^{3},
$$

while the Brusselator kinetics are given by

$$
f(u, v)=a-(b+1) u+u^{2} v, \quad g(u, v)=b u-u^{2} v ; \quad a>0, \quad 0<b<a^{2}+1 .
$$

To validate our weakly nonlinear theory, a combination of numerical bifurcation analysis, for the computation of global bifurcation branches, and full time-dependent PDE numerical simulations are employed. Classical values for the parameters $a$ and $b$ are used. For the Schnakenberg kinetics, these are $a=0.1$ and $b=0.9$ (cf. [20]). For the Brusselator kinetics, $a=3$ will be taken, while different values of $b$, all with $b<a^{2}+1$, will be considered. For these parameters values, the uncoupled bulk-surface system without surface diffusion admits a unique stable steady state and no patterns appear. In this way, the patterns observed arise from the coupling between the bulk and surface. We remark that a symmetry-breaking bifurcation mechanism for particular forms of the nonlinearities has also been explored in [22] and [20] through full PDE simulations. 
The outline of this paper is as follows. In $\S 2$, for arbitrary reaction kinetics, we derive amplitude equations (normal forms) near either a Hopf, a pitchfork, or a pitchfork-Hopf, bifurcation point of the linearization of the base state. In $\S 3$, we analyze these normal forms and interpret their equilibria in terms of limit cycles or Turing-type patterns of the coupled original system. Subsections 3.1 and 3.2, respectively, treat separately the codimension-one and the codimensiontwo cases. Numerical validation of the weakly nonlinear theory with the classical Schnakenberg and Brusselator reaction kinetics is provided in $\S 4$. $\S 5$ is distinct from the previous sections in that, through PDE simulations, it gives a glimpse into novel nonlinear patterns that can occur for the coupled system away from bifurcation points. In particular, the dynamics and formation of rotating waves is explored for a coupled bulk-surface reaction-diffusion system with a slightly more general boundary condition than (1.3). Finally, in $\S 6$, we briefly summarize the paper and discuss a few open problems that warrant further study.

\section{Weakly nonlinear theory}

In this section, the method of multiple time-scales is used to derive amplitude equations describing the branching behavior near three distinct bifurcations:

- Trivial mode $n=0$ undergoes a Hopf bifurcation, at which the bifurcating solution is invariant under rotation and reflection symmetries.

- Nontrivial mode $\{n,-n\} \in \mathbb{Z} \backslash\{0\}$ loses stability through a pitchfork bifurcation, at which the bifurcating solutions are equivariant under rotation and reflection symmetries.

- Pitchfork-Hopf (Turing-Hopf), when the previous two bifurcations occur simultaneously.

From the linear stability analysis, curves of codimension-one bifurcation points and their codimension-two intersection have been computed in the plane of parameters $\left(K_{v}, D_{v}\right)$ (see Fig. 1 and Fig. 2). This motivates introducing a twoparameter bifurcation analysis.

\subsection{Preliminaries}

Before formulating the multiple time-scale asymptotic expansion, the coupled bulk-surface system (1.1)-(1.4) is rewritten as an evolution equation, which then facilitates below the introduction of an extended linear operator and its adjoint:

$$
\dot{W}=\boldsymbol{F}(W)=\left(\begin{array}{c}
D_{u} \Delta U-\sigma_{u} U \\
D_{v} \Delta V-\sigma_{v} V \\
\frac{d_{u}}{R^{2}} u_{\theta \theta}-K_{u}(u-U)+f(u, v) \\
\frac{d_{v}}{R^{2}} v_{\theta \theta}-K_{v}(v-V)+g(u, v)
\end{array}\right)
$$

Here $\boldsymbol{F}$ is a nonlinear functional acting on $\mathcal{W}$, defined as a space of vector functions whose components satisfy the appropriate Langmuir boundary conditions:

$$
\mathcal{W}=\left\{W=\left(\begin{array}{c}
U(r, \theta) \\
V(r, \theta) \\
u(\theta) \\
v(\theta)
\end{array}\right) \mid \begin{array}{l}
\left.D_{u} \partial_{r} U\right|_{r=R}=K_{u}\left(u-\left.U\right|_{r=R}\right) \\
\left.D_{v} \partial_{r} V\right|_{r=R}=K_{v}\left(v-\left.V\right|_{r=R}\right)
\end{array}\right\}
$$

The radially symmetric steady state (i.e. the base state) for (2.1) is given by

$$
W_{e}=\left(\begin{array}{cl}
A_{0}(0) \frac{I_{0}\left(\omega_{u} r\right)}{I_{0}\left(\omega_{u} R\right)} e_{1}^{T} \boldsymbol{u}_{e} \\
B_{0}(0) \frac{I_{0}\left(\omega_{v} r\right)}{I_{0}\left(\omega_{v} R\right)} e_{2}^{T} \boldsymbol{u}_{e} \\
\boldsymbol{u}_{e}
\end{array}\right), \quad \begin{cases}\omega_{u}=\sqrt{\frac{\sigma_{u}}{D_{u}}}, & A_{0}(0)=\frac{K_{u} I_{0}\left(\omega_{u} R\right)}{D_{u} \omega_{u} I_{0}^{\prime}\left(\omega_{u} R\right)+K_{u} I_{0}\left(\omega_{u} R\right)} \\
\omega_{v}=\sqrt{\frac{\sigma_{v}}{D_{v}}}, & B_{0}(0)=\frac{K_{v} I_{0}\left(\omega_{v} R\right)}{D_{v} \omega_{v} I_{0}^{\prime}\left(\omega_{v} R\right)+K_{v} I_{0}\left(\omega_{v} R\right)}\end{cases}
$$

where $\boldsymbol{u}_{e}=\left(u_{e}, v_{e}\right)^{T}$ is the surface steady state vector satisfying the nonlinear algebraic equation

$$
\left\{\begin{array}{l}
K_{u} p_{0}(0) u_{e}-f\left(u_{e}, v_{e}\right)=0 \\
K_{v} q_{0}(0) v_{e}-g\left(u_{e}, v_{e}\right)=0
\end{array}, \quad\left\{\begin{array}{l}
p_{0}(0)=\frac{D_{u} \omega_{u} I_{0}^{\prime}\left(\omega_{u} R\right)}{D_{u} \omega_{u} I_{0}^{\prime}\left(\omega_{u} R\right)+K_{u} I_{0}\left(\omega_{u} R\right)} \\
q_{0}(0)=\frac{D_{v} \omega_{v} I_{0}^{\prime}\left(\omega_{v} R\right)}{D_{v} \omega_{v} I_{0}^{\prime}\left(\omega_{v} R\right)+K_{v} I_{0}\left(\omega_{v} R\right)}
\end{array}\right.\right.
$$

Here $I_{n}(z)$ for $n \in \mathbb{Z}$ are the usual modified Bessel functions. Next, by expanding the nonlinear functional about the base state, we get

$$
\dot{W}=\underbrace{\boldsymbol{F}\left(W_{e}\right)}_{=0}+\mathcal{L}\left(W-W_{e}\right)+\mathcal{B}\left(W-W_{e}, W-W_{e}\right)+\mathcal{C}\left(W-W_{e}, W-W_{e}, W-W_{e}\right)+\ldots,
$$

where $\mathcal{L}$ is the linearized operator defined by

$$
\mathcal{L}(W)=\left(\begin{array}{c}
D_{u} \Delta U-\sigma_{u} U \\
D_{v} \Delta V-\sigma_{v} V \\
\frac{d_{u}}{R^{2}} u_{\theta \theta}-K_{u}(u-U)+f_{u}^{e} u+f_{v}^{e} v \\
\frac{d_{v}}{R^{2}} v_{\theta \theta}-K_{v}(v-V)+g_{u}^{e} u+g_{v}^{e} v
\end{array}\right)
$$


while $\mathcal{B}$ and $\mathcal{C}$ are, respectively, bilinear and trilinear forms. For each $n \in \mathbb{Z}$, the eigenfunctions for the linearized operator are given by

$$
\mathcal{W}_{n}=\left(\begin{array}{c}
A_{n}(\lambda) \frac{I_{n}\left(\Omega_{u} r\right)}{I_{n}\left(\Omega_{u} R\right)} e_{1}^{T} \phi_{n} \\
B_{n}(\lambda) \frac{I_{n}\left(\Omega_{v} r\right)}{I_{n}\left(\Omega_{v} R\right)} e_{2}^{T} \phi_{n} \\
\phi_{n}
\end{array}\right) e^{i n \theta}, \quad\left\{\begin{array}{ll}
\Omega_{u}=\sqrt{\frac{\lambda+\sigma_{u}}{D_{u}}}, & A_{n}(\lambda)=\frac{K_{u} I_{n}\left(\Omega_{u} R\right)}{D_{u} \Omega_{u} I_{n}^{\prime}\left(\Omega_{u} R\right)+K_{u} I_{n}\left(\Omega_{u} R\right)} \\
\Omega_{v}=\sqrt{\frac{\lambda+\sigma_{v}}{D_{v}}}, & B_{n}(\lambda)=\frac{K_{v} I_{n}\left(\Omega_{v} R\right)}{D_{v} \Omega_{v} I_{n}^{\prime}\left(\Omega_{v} R\right)+K_{v} I_{n}\left(\Omega_{v} R\right)}
\end{array} .\right.
$$

The eigenvector $\phi_{n}=\left(\phi_{n}, \psi_{n}\right)^{T}$ satisfies a homogeneous linear system

$$
\left[\boldsymbol{\Phi}_{n}(\lambda)\right] \boldsymbol{\phi}_{n}=\left[J_{e}-\lambda I-\left(K_{u} p_{n}(\lambda)+\frac{n^{2} d_{u}}{R^{2}}\right) E_{1}-\left(K_{v} q_{n}(\lambda)+\frac{n^{2} d_{v}}{R^{2}}\right) E_{2}\right] \boldsymbol{\phi}_{n}=\mathbf{0},
$$

with $J_{e}, E_{1}, E_{2}, p_{n}(\lambda)$, and $q_{n}(\lambda)$ defined as

$$
J_{e}=\left(\begin{array}{ll}
f_{u}^{e} & f_{v}^{e} \\
g_{u}^{e} & g_{v}^{e}
\end{array}\right), \quad E_{i}=e_{i} e_{i}^{T} \in \mathbb{R}^{2 \times 2}, \quad\left\{\begin{array}{l}
p_{n}(\lambda)=\frac{D_{u} \Omega_{u} I_{n}^{\prime}\left(\Omega_{u} R\right)}{D_{u} \Omega_{u} I_{n}^{\prime}\left(\Omega_{u} R\right)+K_{u} I_{n}\left(\Omega_{u} R\right)}=1-A_{n}(\lambda) \\
q_{n}(\lambda)=\frac{D_{v} \Omega_{v} I_{n}^{\prime}\left(\Omega_{v} R\right)}{D_{v} \Omega_{v} I_{n}^{\prime}\left(\Omega_{v} R\right)+K_{v} I_{n}\left(\Omega_{v} R\right)}=1-B_{n}(\lambda)
\end{array}\right.
$$

Here, the vectors $e_{1}$ and $e_{2}$ form the standard orthonormal basis in the phase space defined by the species $u$ and $v$. A nontrivial solution to system (2.8) will exist when the following transcendental equation is satisfied:

$$
F_{n}(\lambda)=\operatorname{det}\left[\Phi_{n}(\lambda)\right]=0
$$

In the multi-scale analysis below, an application of the solvability condition requires the formulation of an adjoint linear operator $\mathcal{L}^{\star}$, defined by

$$
\mathcal{L}^{\star}\left(W^{\star}\right)=\left(\begin{array}{c}
D_{u} \Delta U^{\star}-\sigma_{u} U^{\star} \\
D_{v} \Delta V^{\star}-\sigma_{v} V^{\star} \\
\frac{d_{u}}{R^{2}} u_{\theta \theta}^{\star}-K_{u}\left(u^{\star}-U^{\star}\right)+f_{u}^{e} u^{\star}+g_{u}^{e} v^{\star} \\
\frac{d_{v}}{R^{2}} v_{\theta \theta}^{\star}-K_{v}\left(v^{\star}-V^{\star}\right)+f_{v}^{e} u^{\star}+g_{v}^{e} v^{\star}
\end{array}\right)
$$

For the special case of Langmuir boundary conditions, the dual space satisfies $\mathcal{W}^{\star}=\mathcal{W}$, which means that both the boundary conditions and their adjoint are identical. Furthermore, the adjoint eigenfunctions yield

$$
\mathcal{W}_{n}^{\star}=\left(\begin{array}{c}
\overline{A_{n}(\lambda)} \frac{I_{n}\left(\overline{\Omega_{u}} r\right)}{I_{n}\left(\overline{\Omega_{u}} R\right)} e_{1}^{T} \phi_{n}^{\star} \\
\overline{B_{n}(\lambda)} \frac{I_{n}\left(\overline{\Omega_{v}} r\right)}{I_{n}\left(\overline{\Omega_{v}} R\right)} e_{2}^{T} \phi_{n}^{\star} \\
\phi_{n}^{\star}
\end{array}\right) e^{i n \theta}
$$

It is then readily verified that $\mathcal{W}_{n}$ and $\mathcal{W}_{n}^{\star}$ form an orthogonal set of eigenfunctions, satisfying

$$
\left\langle\mathcal{W}_{m}^{\star}, \mathcal{W}_{n}\right\rangle=0, \quad \text { if } m \neq n
$$

where the inner-product in (2.13) is defined by

$$
\left\langle W^{\star}, W\right\rangle=\int_{0}^{2 \pi} \int_{0}^{R}\left[\overline{U^{\star}} U+\overline{V^{\star}} V\right] r d r d \theta+\int_{0}^{2 \pi}\left[\overline{u^{\star}} u+\overline{v^{\star}} v\right] R d \theta .
$$

The set of eigenfunctions can be normalized so that $\left\langle\mathcal{W}_{n}^{\star}, \mathcal{W}_{n}\right\rangle=1$ for all $n$. Finally, using the previous definitions of linear operators, eigenfunctions and inner-product, one may easily verify that the following properties hold:

$$
\mathcal{L} \mathcal{W}_{n}=\lambda \mathcal{W}_{n}, \quad \mathcal{L}^{\star} \mathcal{W}_{n}^{\star}=\bar{\lambda} \mathcal{W}_{n}^{\star}, \quad\left\langle W^{\star}, \mathcal{L} W\right\rangle=\left\langle\mathcal{L}^{\star} W^{\star}, W\right\rangle
$$

Lastly, we define more precisely the bilinear and trilinear forms that arise in the expansion (2.5). They are defined by

$$
\mathcal{B}\left(W_{j}, W_{k}\right)=\left(\begin{array}{c}
0 \\
0 \\
B\left(\boldsymbol{u}_{j}, \boldsymbol{u}_{k}\right)
\end{array}\right), \quad \mathcal{C}\left(W_{j}, W_{k}, W_{l}\right)=\left(\begin{array}{c}
0 \\
0 \\
C\left(\boldsymbol{u}_{j}, \boldsymbol{u}_{k}, \boldsymbol{u}_{l}\right)
\end{array}\right)
$$

The first two components of $\mathcal{B}$ and $\mathcal{C}$ vanish since the diffusion process occurring in the bulk is linear. The reduced bilinear and trilinear forms $B$ and $C$ are defined by

$$
B\left(\boldsymbol{u}_{j}, \boldsymbol{u}_{k}\right)=\frac{1}{2}\left(I \otimes \boldsymbol{u}_{k}^{T}\right) H_{e} \boldsymbol{u}_{j}, \quad C\left(\boldsymbol{u}_{j}, \boldsymbol{u}_{k}, \boldsymbol{u}_{l}\right)=\frac{1}{6}\left(I \otimes \boldsymbol{u}_{l}^{T}\right) T_{e}\left(\boldsymbol{u}_{j} \otimes \boldsymbol{u}_{k}\right),
$$

where $\otimes$ is the Kronecker product and $H_{e}, T_{e}$ are matrices involving the second and third order partial derivatives

$$
H_{e}=\left(\begin{array}{ll}
f_{u u}^{e} & f_{u v}^{e} \\
f_{u v}^{e} & f_{v v}^{e} \\
g_{u u}^{e} & g_{u v}^{e} \\
g_{u v}^{e} & g_{v v}^{e}
\end{array}\right), \quad T_{e}=\left(\begin{array}{llll}
f_{u u u}^{e} & f_{u u v}^{e} & f_{u u v}^{e} & f_{u v v}^{e} \\
f_{u u v}^{e} & f_{u v v}^{e} & f_{u v v}^{e} & f_{v v v}^{e} \\
g_{u u u}^{e} & g_{u u v}^{e} & g_{u u v}^{e} & g_{u v v}^{e} \\
g_{u u v}^{e} & g_{u v v}^{e} & g_{u v v}^{e} & g_{v v v}^{e}
\end{array}\right)
$$




\subsection{Multi-scale expansion}

Let $\mu=\left(K_{v}, D_{v}\right)^{T}$ denote the vector of bifurcation parameters. As usual, a slow time-scale $\tau=\varepsilon^{2} t$, with $\varepsilon \ll 1$, is introduced. Using the same scaling, the parameters are slightly perturbed,

$$
\mu=\mu_{0}+\varepsilon^{2} \mu_{1}, \quad\left\|\mu_{1}\right\|=1
$$

Here $\mu_{0}$ is the bifurcation point and $\mu_{1}$ is a unit vector indicating the direction of the bifurcation. The full system is then expanded in a regular asymptotic power series around the base state as

$$
W=W_{e}+\varepsilon W_{1}+\varepsilon^{2} W_{2}+\varepsilon^{3} W_{3}+\mathcal{O}\left(\varepsilon^{4}\right),
$$

where the subscript here refers to the expansion order rather than the mode of the eigenfunction. Next, by inserting (2.17) and (2.18) into (2.5), and collecting powers of $\varepsilon$ we obtain that

$$
\begin{aligned}
& \varepsilon \partial_{t} W_{1}+\varepsilon^{2} \partial_{t} W_{2}+\varepsilon^{3}\left(\partial_{t} W_{3}+\partial_{\tau} W_{1}\right)=\varepsilon \mathcal{L} W_{1}+\varepsilon^{2}\left(\mathcal{L} W_{2}+\mathcal{B}\left(W_{1}, W_{1}\right)+\left(\begin{array}{c}
0 \\
\omega_{v}^{2} V_{e}(r) e_{2}^{T} \mu_{1} \\
0 \\
-q_{0}(0) v_{e} e_{1}^{T} \mu_{1}
\end{array}\right)\right) \\
& +\varepsilon^{3}\left(\mathcal{L} W_{3}+2 \mathcal{B}\left(W_{1}, W_{2}\right)+\mathcal{C}\left(W_{1}, W_{1}, W_{1}\right)+\left(\begin{array}{c}
0 \\
\frac{\partial_{t}+\sigma_{v}}{D_{v 0}} V_{1} e_{2}^{T} \mu_{1} \\
0 \\
-\left(v_{1}-\left.V_{1}\right|_{r=R}\right) e_{1}^{T} \mu_{1}
\end{array}\right)\right)+\mathcal{O}\left(\varepsilon^{4}\right)
\end{aligned}
$$

where the vectors $e_{1}$ and $e_{2}$ are now the standard orthonormal basis in the parameter space defined by $K_{v}$ and $D_{v}$. The perturbed boundary conditions satisfy

$$
\begin{array}{r}
\sum_{j=1}^{3} \varepsilon^{j}\left(D_{u} \partial_{r} U_{j}+K_{u} U_{j}-K_{u} u_{j}\right)+\mathcal{O}\left(\varepsilon^{4}\right)=0, \quad r=R . \\
\sum_{j=1}^{3} \varepsilon^{j}\left(D_{v 0} \partial_{r} V_{j}+K_{v 0} V_{j}-K_{v 0} v_{j}\right)-\left(\varepsilon^{2} q_{0}(0) v_{e}+\varepsilon^{3}\left(v_{1}-V_{1}\right)\right) \beta^{T} \mu_{1}+\mathcal{O}\left(\varepsilon^{4}\right)=0, \quad r=R,
\end{array}
$$

where the vector $\beta$ is defined by

$$
\beta=\left(\begin{array}{c}
1 \\
-K_{v 0} / D_{v 0}
\end{array}\right)
$$

\subsection{Weakly nonlinear analysis of patterns}

The leading order solution corresponds to the base state defined by (2.3) evaluated at the bifurcation point $\mu=\mu_{0}$. Next, by collecting terms at $\mathcal{O}(\varepsilon)$ we get the linearized problem

$$
\partial_{t} W_{1}=\mathcal{L}\left(\mu_{0} ; W_{1}\right), \quad\left\{\begin{array}{l}
D_{u} \partial_{r} U_{1}=K_{u}\left(u_{1}-U_{1}\right) \\
D_{v 0} \partial_{r} V_{1}=K_{v 0}\left(v_{1}-V_{1}\right)
\end{array} \quad, \quad r=R .\right.
$$

Here the notation $\mathcal{L}\left(\mu_{0} ; \cdot\right)$ indicates that the linear operator is evaluated at the bifurcation point. The solution of the linearized system depends on the type of bifurcation and the spatial mode considered. We will consider the following three cases:

- Hopf bifurcation. The critical eigenvalues and spatial mode are respectively $\lambda= \pm i \lambda_{I}$ and $n=0$, which yields

$$
W_{1}=\mathcal{W}_{0} A_{0}(\tau) e^{i \lambda_{I} t}+\overline{\mathcal{W}_{0}} \overline{A_{0}(\tau)} e^{-i \lambda_{I} t},
$$

where the eigenfunction $\mathcal{W}_{0}$ is evaluated at $\mu_{0}$ and $\lambda=i \lambda_{I}$.

- Pitchfork bifurcation. The critical eigenvalue is $\lambda=0$ and because of the reflection symmetry, if $n \neq 0$ is a critical spatial mode, then so is $-n$. Moreover, it is known that the center manifold preserves the symmetries of the system. Therefore, the center eigenspace and manifold are also two-dimensional, and the solution in the linear regime has the form

$$
W_{1}=\mathcal{W}_{n} A_{n}(\tau)+\mathcal{W}_{-n} \overline{A_{n}(\tau)},
$$

where $\mathcal{W}_{ \pm n}$ are evaluated at $\mu_{0}$ and $\lambda=0$.

- Pitchfork-Hopf bifurcation. The critical spatial modes are $\{0, n,-n\}$, with $n \neq 0$, and the bifurcating eigenvalues of the linearized problem are $\left\{ \pm i \lambda_{I}, 0\right\}$. This yields a four-dimensional center eigenspace of the form

$$
W_{1}=\mathcal{W}_{0} A_{0}(\tau) e^{i \lambda_{I} t}+\overline{\mathcal{W}_{0}} \overline{A_{0}(\tau)} e^{-i \lambda_{I} t}+\mathcal{W}_{n} A_{n}(\tau)+\mathcal{W}_{-n} \overline{A_{n}(\tau)}
$$

Again, all the eigenfunctions above are evaluated at the bifurcation point and the critical set of eigenvalues. 
The goal of our analysis is to derive evolution equations for the complex amplitudes $A_{0}$ and $A_{n}$, where the subscript here indicates which spatial mode has exchanged stability through the bifurcation.

By collecting terms of order $\mathcal{O}\left(\epsilon^{2}\right)$ in $(2.19)$, we obtain

$$
\partial_{t} W_{2}=\mathcal{L}\left(\mu_{0} ; W_{2}\right)+\mathcal{B}\left(W_{1}, W_{1}\right)+\left(\begin{array}{c}
0 \\
\omega_{v}^{2} V_{e}(r) e_{2}^{T} \mu_{1} \\
0 \\
-q_{0}(0) v_{e} e_{1}^{T} \mu_{1}
\end{array}\right)
$$

together with the appropriate boundary conditions, as obtained from (2.20):

$$
\left\{\begin{array}{l}
D_{u} \partial_{r} U_{2}=K_{u}\left(u_{2}-U_{2}\right) \\
D_{v 0} \partial_{r} V_{2}=K_{v 0}\left(v_{2}-V_{2}\right)+q_{0}(0) v_{e} \beta^{T} \mu_{1}
\end{array} \quad, \quad r=R .\right.
$$

The evaluation of the quadratic terms $\mathcal{B}\left(W_{1}, W_{1}\right)$ will depend on whether we consider the bifurcations (2.23), (2.24) or (2.25). Below, the nontrivial part of the bilinear form is stated explicitly for each case.

- Hopf bifurcation:

$$
B\left(\boldsymbol{u}_{1}, \boldsymbol{u}_{1}\right)=A_{0}^{2} B\left(\phi_{0}, \phi_{0}\right) e^{2 i \lambda_{I} t}+2\left|A_{0}\right|^{2} B\left(\phi_{0}, \overline{\phi_{0}}\right)+{\overline{A_{0}}}^{2} B\left(\overline{\phi_{0}}, \overline{\phi_{0}}\right) e^{-2 i \lambda_{I} t}
$$

- Pitchfork bifurcation:

$$
B\left(\boldsymbol{u}_{1}, \boldsymbol{u}_{1}\right)=A_{n}^{2} B\left(\boldsymbol{\phi}_{n}, \boldsymbol{\phi}_{n}\right) e^{2 i n \theta}+2\left|A_{n}\right|^{2} B\left(\boldsymbol{\phi}_{n}, \boldsymbol{\phi}_{-n}\right)+{\overline{A_{n}}}^{2} B\left(\boldsymbol{\phi}_{-n}, \boldsymbol{\phi}_{-n}\right) e^{-2 i n \theta} .
$$

- Pitchfork-Hopf bifurcation:

$$
\begin{aligned}
B\left(\boldsymbol{u}_{1}, \boldsymbol{u}_{1}\right) & =A_{0}^{2} B\left(\boldsymbol{\phi}_{0}, \boldsymbol{\phi}_{0}\right) e^{2 i \lambda_{I} t}+2\left|A_{0}\right|^{2} B\left(\boldsymbol{\phi}_{0}, \overline{\boldsymbol{\phi}_{0}}\right)+{\overline{A_{0}}}^{2} B\left(\overline{\boldsymbol{\phi}_{0}}, \overline{\boldsymbol{\phi}_{0}}\right) e^{-2 i \lambda_{I} t} \\
& +A_{n}^{2} B\left(\boldsymbol{\phi}_{n}, \boldsymbol{\phi}_{n}\right) e^{2 i n \theta}+2\left|A_{n}\right|^{2} B\left(\boldsymbol{\phi}_{n}, \boldsymbol{\phi}_{-n}\right)+{\overline{A_{n}}}^{2} B\left(\boldsymbol{\phi}_{-n}, \boldsymbol{\phi}_{-n}\right) e^{-2 i n \theta} \\
& +2 A_{0} A_{n} B\left(\boldsymbol{\phi}_{0}, \boldsymbol{\phi}_{n}\right) e^{i\left(n \theta+\lambda_{I} t\right)}+2 A_{0} \overline{A_{n}} B\left(\boldsymbol{\phi}_{0}, \boldsymbol{\phi}_{-n}\right) e^{i\left(-n \theta+\lambda_{I} t\right)} \\
& +2 \overline{A_{0}} A_{n} B\left(\overline{\boldsymbol{\phi}_{0}}, \boldsymbol{\phi}_{n}\right) e^{i\left(n \theta-\lambda_{I} t\right)}+2 \overline{A_{0} A_{n}} B\left(\overline{\boldsymbol{\phi}_{0}}, \boldsymbol{\phi}_{-n}\right) e^{-i\left(n \theta+\lambda_{I} t\right)}
\end{aligned}
$$

Once again, because of the reflection symmetry, we have that $\phi_{n}=\phi_{-n}$. By examining these bilinear forms, the following ansatz can be formulated for the solution of the system $(2.26)$ :

- Hopf bifurcation:

$$
W_{2}=W_{0000}+A_{0}^{2} W_{2000} e^{2 i \lambda_{I} t}+\left|A_{0}\right|^{2} W_{1100}+{\overline{A_{0}}}^{2} W_{0200} e^{-2 i \lambda_{I} t}
$$

- Pitchfork bifurcation:

$$
W_{2}=W_{0000}+A_{n}^{2} W_{0020}+\left|A_{n}\right|^{2} W_{0011}+{\overline{A_{n}}}^{2} W_{0002}
$$

- Pitchfork-Hopf bifurcation:

$$
\begin{aligned}
W_{2} & =W_{0000}+A_{0}^{2} W_{2000} e^{2 i \lambda_{I} t}+\left|A_{0}\right|^{2} W_{1100}+{\overline{A_{0}}}^{2} W_{0200} e^{-2 i \lambda_{I} t} \\
& +A_{n}^{2} W_{0020}+\left|A_{n}\right|^{2} W_{0011}+{\overline{A_{n}}}^{2} W_{0002} \\
& +A_{0} A_{n} W_{1010} e^{i \lambda_{I} t}+A_{0} \overline{A_{n}} W_{1001} e^{i \lambda_{I} t}+\overline{A_{0}} A_{n} W_{0110} e^{-i \lambda_{I} t}+\overline{A_{0} A_{n}} W_{0101} e^{-i \lambda_{I} t}
\end{aligned}
$$

Next, we briefly outline the computation of the term $W_{0000}$. This term arises from the perturbation of the bifurcation parameters within the base state, and satisfies

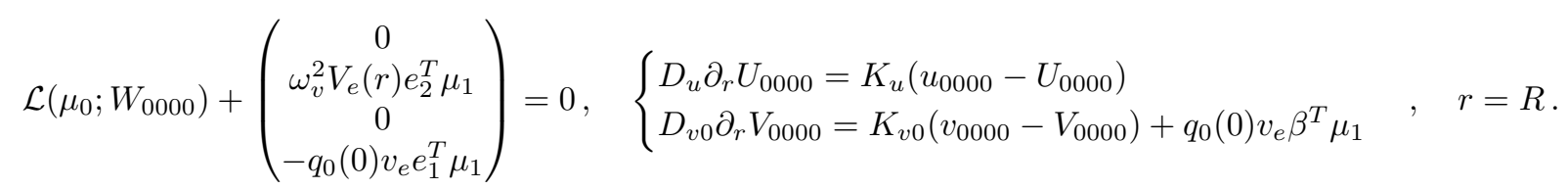

Solving for $U_{0000}$, one obtains the same expression as for the steady state profile, given by

$$
U_{0000}=A_{0}(0) \frac{I_{0}\left(\omega_{u} r\right)}{I_{0}\left(\omega_{u} R\right)} e_{1}^{T} \boldsymbol{u}_{0000}
$$

Since the equation for $V_{0000}$ is forced by a multiple of the steady state solution, the reduction of order method is used to yield the following ansatz:

$$
V_{0000}=\left(\gamma_{0}+\gamma_{1}(r)\right) \frac{I_{0}\left(\omega_{v} r\right)}{I_{0}\left(\omega_{v} R\right)}, \quad \text { with } \quad \gamma_{1}(0)=0
$$


where $\gamma_{1}(r)$ is found to satisfy the second-order differential equation

$$
\gamma_{1}^{\prime \prime}(r) I_{0}\left(\omega_{v} r\right)+\gamma_{1}^{\prime}(r)\left(2 \omega_{v} I_{1}\left(\omega_{v} r\right)+\frac{1}{r} I_{0}\left(\omega_{v} r\right)\right)+\frac{\omega_{v}}{D_{v 0}} e_{2}^{T} \mu_{1} B_{0}(0) I_{0}\left(\omega_{v} r\right) v_{e}=0
$$

The ODE (2.37) is readily solved using $r I_{0}\left(\omega_{v} r\right)$ as an integrating factor. By integrating twice, we obtain that

$$
\gamma_{1}(r)=-\frac{e_{2}^{T} \mu_{1} B_{0}(0) v_{e}}{D_{v 0}} \int_{0}^{\omega_{v} r} \rho\left[1-\left(\frac{I_{1}(\rho)}{I_{0}(\rho)}\right)^{2}\right] d \rho
$$

Then, upon application of the perturbed boundary condition, we determine the constant $\gamma_{0}$ as

$$
\gamma_{0}=B_{0}(0) v_{0000}+\beta^{T} \mu_{1} \frac{q_{0}(0) B_{0}(0)}{K_{v 0}} v_{e}-\gamma_{1}(R)-\frac{D_{v 0} B_{0}(0)}{K_{v 0}} \gamma_{1}^{\prime}(R) .
$$

Next, the evaluation of $U_{0000}$ and $V_{0000}$ on the boundary leads to

$$
\left.\left(\begin{array}{c}
U_{0000} \\
V_{0000}
\end{array}\right)\right|_{r=R}=\left(\begin{array}{c}
A_{0}(0) u_{0000} \\
B_{0}(0) v_{0000}+\Delta^{T} \mu_{1}
\end{array}\right)
$$

where the coefficient of the detuning vector is given explicitly by

$$
\Delta=\frac{q_{0}(0) B_{0}(0)}{K_{v 0}} e_{1}+\frac{\omega_{v}^{2} K_{v 0} R\left(I_{0}^{2}\left(\omega_{v} R\right)-I_{1}^{2}\left(\omega_{v} R\right)\right) / 2-\omega_{v} K_{v 0} I_{0}\left(\omega_{v} R\right) I_{1}\left(\omega_{v} R\right)}{\left(D_{v 0} \omega_{v} I_{1}\left(\omega_{v} R\right)+K_{v 0} I_{0}\left(\omega_{v} R\right)\right)^{2}} e_{2} .
$$

Finally, the substitution of (2.40) into the constraint (2.34) for the membrane components determines $\boldsymbol{u}_{0000}$ as

$$
\boldsymbol{\Phi}_{0}\left(\mu_{0} ; 0\right) \boldsymbol{u}_{0000}=\alpha^{T} \mu_{1} E_{2} \boldsymbol{u}_{e} \quad \Rightarrow \quad \boldsymbol{u}_{0000}=\alpha^{T} \mu_{1}\left[\mathbf{\Phi}_{0}\left(\mu_{0} ; 0\right)\right]^{-1} E_{2} \boldsymbol{u}_{e}
$$

Here the vector coefficient $\alpha$ is defined by

$$
\begin{aligned}
\alpha & =q_{0}(0) e_{1}-K_{v 0} \Delta \\
& =\left(q_{0}(0)\right)^{2} e_{1}+\frac{\omega_{v} K_{v 0}^{2}}{\left(D_{v 0} \omega_{v} I_{1}\left(\omega_{v} R\right)+K_{v 0} I_{0}\left(\omega_{v} R\right)\right)^{2}}\left(I_{0}\left(\omega_{v} R\right) I_{1}\left(\omega_{v} R\right)+\frac{\omega_{v} R}{2}\left(I_{1}^{2}\left(\omega_{v} R\right)-I_{0}^{2}\left(\omega_{v} R\right)\right)\right) e_{2} .
\end{aligned}
$$

Many of the nontrivial $W_{j k l m}$ can be found using the spatial and temporal reflection symmetries of the reduced system. Here, the linear inhomogeneous problems to be solved are listed below.

Starting with the Hopf bifurcation, where $W_{0200}=\overline{W_{2000}}$, it is readily found that $W_{2000}$ satisfies

$$
\mathcal{L}\left(\mu_{0} ; W_{2000}\right)-2 i \lambda_{I} W_{2000}=-\mathcal{B}\left(\mathcal{W}_{0}, \mathcal{W}_{0}\right) \quad \Rightarrow \quad W_{2000}=\left(\begin{array}{c}
A_{0}\left(2 i \lambda_{I}\right) \frac{I_{0}\left(\Omega_{2 u} r\right)}{I_{0}\left(\Omega_{2 u} R\right)} e_{1}^{T} \boldsymbol{u}_{2000} \\
B_{0}\left(2 i \lambda_{I}\right) \frac{I_{0}\left(\Omega_{2 v} r\right)}{I_{0}\left(\Omega_{2 v} R\right)} e_{2}^{T} \boldsymbol{u}_{2000} \\
\boldsymbol{u}_{2000}
\end{array}\right)
$$

where $\Omega_{2 u}$ and $\Omega_{2 v}$ are defined by

$$
\Omega_{2 u}=\sqrt{\frac{\sigma_{u}+2 i \lambda_{I}}{D_{u}}}, \quad \Omega_{2 v}=\sqrt{\frac{\sigma_{v}+2 i \lambda_{I}}{D_{v}}} .
$$

Next, solving for $W_{1100}$ leads to

$$
\mathcal{L}\left(\mu_{0} ; W_{1100}\right)=-2 \mathcal{B}\left(\mathcal{W}_{0}, \overline{\mathcal{W}_{0}}\right) \quad \Rightarrow \quad W_{1100}=\left(\begin{array}{c}
A_{0}(0) \frac{I_{0}\left(\omega_{u} r\right)}{I_{0}\left(\omega_{u} R\right)} e_{1}^{T} \boldsymbol{u}_{1100} \\
B_{0}(0) \frac{I_{0}\left(\omega_{v} r\right)}{I_{0}\left(\omega_{v} R\right)} e_{2}^{T} \boldsymbol{u}_{1100} \\
\boldsymbol{u}_{1100}
\end{array}\right) .
$$

Finally, $\boldsymbol{u}_{2000}$ and $\boldsymbol{u}_{1100}$ each satisfy the following two-dimensional linear systems:

$$
\left[\boldsymbol{\Phi}_{0}\left(\mu_{0} ; 2 i \lambda_{I}\right)\right] \boldsymbol{u}_{2000}=-B\left(\phi_{0}, \phi_{0}\right), \quad\left[\boldsymbol{\Phi}_{0}\left(\mu_{0} ; 0\right)\right] \boldsymbol{u}_{1100}=-2 B\left(\phi_{0}, \overline{\phi_{0}}\right) .
$$

With regards to the pitchfork bifurcation, reflection symmetry guarantees that $W_{0002}=\overline{W_{0020}}$. Hence, the systems to be solved and their solutions are given by

$$
\mathcal{L}\left(\mu_{0} ; W_{0020}\right)=-\mathcal{B}\left(\mathcal{W}_{n}, \mathcal{W}_{n}\right) \quad \Rightarrow \quad W_{0020}=\left(\begin{array}{c}
A_{2 n}(0) \frac{I_{2 n}\left(\omega_{u} r\right)}{I_{2 n}\left(\omega_{u} R\right)} e_{1}^{T} \boldsymbol{u}_{0020} \\
B_{2 n}(0) \frac{I_{2 n}\left(\omega_{v} r\right)}{I_{2 n}\left(\omega_{v} R\right)} e_{2}^{T} \boldsymbol{u}_{0020} \\
\boldsymbol{u}_{0020}
\end{array}\right) e^{2 i n \theta}
$$

and

$$
\mathcal{L}\left(\mu_{0} ; W_{0011}\right)=-2 \mathcal{B}\left(\mathcal{W}_{n}, \mathcal{W}_{-n}\right) \quad \Rightarrow \quad W_{0011}=\left(\begin{array}{c}
A_{0}(0) \frac{I_{0}\left(\omega_{u} r\right)}{I_{0}\left(\omega_{u} R\right)} e_{1}^{T} \boldsymbol{u}_{0011} \\
B_{0}(0) \frac{I_{0}\left(\omega_{v} r\right)}{I_{0}\left(\omega_{v} R\right)} e_{2}^{T} \boldsymbol{u}_{0011} \\
\boldsymbol{u}_{0011}
\end{array}\right)
$$


Here $\boldsymbol{u}_{0020}$ and $\boldsymbol{u}_{0011}$ satisfy the reduced linear systems

$$
\left[\boldsymbol{\Phi}_{2 n}\left(\mu_{0} ; 0\right)\right] \boldsymbol{u}_{0020}=-B\left(\boldsymbol{\phi}_{n}, \boldsymbol{\phi}_{n}\right), \quad\left[\boldsymbol{\Phi}_{0}\left(\mu_{0} ; 0\right)\right] \boldsymbol{u}_{0011}=-2 B\left(\boldsymbol{\phi}_{n}, \boldsymbol{\phi}_{-n}\right) .
$$

The $W_{j k l m}$ common to both the codimension-one and codimension-two bifurcations remain the same. In addition to those terms, one needs to solve for the mixed coefficients $W_{1010}$ and $W_{1001}$ as follows:

$$
\mathcal{L}\left(\mu_{0} ; W_{1010}\right)-i \lambda_{I} W_{1010}=-2 \mathcal{B}\left(\mathcal{W}_{0}, \mathcal{W}_{n}\right) \quad \Rightarrow \quad W_{1010}=\left(\begin{array}{c}
A_{n}\left(i \lambda_{I}\right) \frac{I_{n}\left(\Omega_{u} r\right)}{I_{n}\left(\Omega_{u} R\right)} e_{1}^{T} \boldsymbol{u}_{1010} \\
B_{n}\left(i \lambda_{I}\right) \frac{I_{n}\left(\Omega_{v} r\right)}{I_{n}\left(\Omega_{v} R\right)} e_{2}^{T} \boldsymbol{u}_{1010} \\
\boldsymbol{u}_{1010}
\end{array}\right) e^{i n \theta}
$$

and

$$
\mathcal{L}\left(\mu_{0} ; W_{1001}\right)-i \lambda_{I} W_{1001}=-2 \mathcal{B}\left(\mathcal{W}_{0}, \mathcal{W}_{-n}\right) \quad \Rightarrow \quad W_{1001}=\left(\begin{array}{c}
A_{n}\left(i \lambda_{I}\right) \frac{I_{n}\left(\Omega_{u} r\right)}{I_{n}\left(\Omega_{u} R\right)} e_{1}^{T} \boldsymbol{u}_{1001} \\
B_{n}\left(i \lambda_{I}\right) \frac{I_{n}\left(\Omega_{v} r\right)}{I_{n}\left(\Omega_{v} R\right)} e_{2}^{T} \boldsymbol{u}_{1001} \\
\boldsymbol{u}_{1001}
\end{array}\right) e^{-i n \theta}
$$

where $\boldsymbol{u}_{1010}=\boldsymbol{u}_{1001}$ (because $\phi_{n}=\phi_{-n}$ ), which satisfies the further two-dimensional linear system

$$
\left[\boldsymbol{\Phi}_{n}\left(\mu_{0} ; i \lambda_{I}\right)\right] \boldsymbol{u}_{1010}=-2 B\left(\phi_{0}, \phi_{n}\right) .
$$

The remaining coefficients are found trivially using symmetries, and are

$$
W_{0101}=\overline{W_{1010}}, \quad W_{0110}=\overline{W_{1001}} .
$$

\subsection{Solvability condition and amplitude equations}

Upon collecting terms of $\mathcal{O}\left(\varepsilon^{3}\right)$ in $(2.19)$, we obtain that

$$
\partial_{t} W_{3}-\mathcal{L}\left(\mu_{0} ; W_{3}\right)=-\partial_{\tau} W_{1}+2 \mathcal{B}\left(W_{1}, W_{2}\right)+\mathcal{C}\left(W_{1}, W_{1}, W_{1}\right)+\left(\begin{array}{c}
0 \\
e_{2}^{T} \mu_{1} \frac{\partial_{t}+\sigma_{v}}{D_{v 0}} V_{1} \\
0 \\
-e_{1}^{T} \mu_{1}\left(v_{1}-\left.V_{1}\right|_{r=R}\right)
\end{array}\right)
$$

while the $\mathcal{O}\left(\varepsilon^{3}\right)$ terms in the boundary conditions (2.20) yield

$$
\left.\partial_{r}\left(\begin{array}{c}
D_{u} U_{3} \\
D_{v 0} V_{3}
\end{array}\right)\right|_{r=R}=\left(\begin{array}{c}
K_{u}\left(u_{3}-\left.U_{3}\right|_{r=R}\right) \\
K_{v 0}\left(v_{3}-\left.V_{3}\right|_{r=R}\right)
\end{array}\right)+\left(\begin{array}{c}
0 \\
\beta^{T} \mu_{1}\left(v_{1}-\left.V_{1}\right|_{r=R}\right)
\end{array}\right) .
$$

Next, suitable ansatzes are formulated based on the method of undetermined coefficients. Nonlinear evolution equations for the amplitudes $A_{0}(\tau)$ and $A_{n}(\tau)$ will arise from the application of a solvability condition on (2.54) and (2.55).

- Hopf bifurcation. Since $n=0$ is the unstable mode, the solution is radially symmetric of the form

$$
W_{3}=X e^{i \lambda_{I} t}+\bar{X} e^{-i \lambda_{I} t}, \quad X=\left(\begin{array}{c}
x_{1}(r) \\
x_{2}(r) \\
x_{3} \\
x_{4}
\end{array}\right)
$$

- Pitchfork bifurcation. The bifurcating branch is stationary and spatially inhomogeneous (i.e. angularly dependent)

$$
W_{3}=Y, \quad Y=\left(\begin{array}{c}
y_{1}(r, \theta) \\
y_{2}(r, \theta) \\
y_{3}(\theta) \\
y_{4}(\theta)
\end{array}\right)
$$

- Pitchfork-Hopf bifurcation. With $X$ and $Y$ defined as in the other cases, we have

$$
W_{3}=X e^{i \lambda_{I} t}+\bar{X} e^{-i \lambda_{I} t}+Y \text {. }
$$

When treating the Hopf bifurcation, equations (2.54) and (2.55) represent a forced oscillatory system. Typically, the presence of forcing with resonant terms generates secular growth of the solution. However, since boundedness of the solution is required on the fast time-scale, these secular terms must be eliminated for self-consistency of the multiple time-scale asymptotic expansion. This elimination is done using a solvability condition.

Upon substituting (2.56) into (2.54), and equating coefficients of $e^{i \lambda_{I} t}$, we get

$$
\begin{aligned}
& i \lambda_{I} X-\mathcal{L}\left(\mu_{0} ; X\right)=-\mathcal{W}_{0} \frac{d A_{0}}{d \tau}+\left(2 \mathcal{B}\left(\mathcal{W}_{0}, W_{0000}\right)+\left(\begin{array}{c}
e_{2}^{T} \mu_{1} \Omega_{v}^{2} B_{0}\left(i \lambda_{I}\right) \frac{I_{0}\left(\Omega_{v} r\right)}{I_{0}\left(\Omega_{v} R\right)} \\
0 \\
-e_{1}^{T} \mu_{1} q_{0}\left(i \lambda_{I}\right)
\end{array}\right) e_{2}^{T} \phi_{0}\right) A_{0} \\
& +\left(2 \mathcal{B}\left(\mathcal{W}_{0}, W_{1100}\right)+2 \mathcal{B}\left(\overline{\mathcal{W}_{0}}, W_{2000}\right)+3 \mathcal{C}\left(\mathcal{W}_{0}, \mathcal{W}_{0}, \overline{\mathcal{W}_{0}}\right)\right)\left|A_{0}\right|^{2} A_{0}
\end{aligned}
$$


where $X$ satisfies the perturbed boundary condition given by

$$
\left[\left.\partial_{r}\left(\begin{array}{c}
D_{u} x_{1} \\
D_{v 0} x_{2}
\end{array}\right)\right|_{r=R}-\left(\begin{array}{c}
K_{u}\left(x_{3}-\left.x_{1}\right|_{r=R}\right) \\
K_{v 0}\left(x_{4}-\left.x_{2}\right|_{r=R}\right)
\end{array}\right)\right]=\left(\begin{array}{c}
0 \\
\beta^{T} \mu_{1} q_{0}\left(i \lambda_{I}\right) e_{2}^{T} \phi_{0}
\end{array}\right) A_{0} .
$$

Since its bifurcating solution is stationary, a different argument must be invoked when treating the pitchfork bifurcation. Again, the substitution of (2.57) into (2.54) leads to

$$
\begin{aligned}
& -\mathcal{L}\left(\mu_{0} ; Y\right)=-\mathcal{W}_{n} \frac{d A_{n}}{d \tau}+\left(2 \mathcal{B}\left(\mathcal{W}_{n}, W_{0000}\right)+\left(\begin{array}{c}
0 \\
e_{2}^{T} \mu_{1} \omega_{v}^{2} B_{n}(0) \frac{I_{n}\left(\omega_{v} r\right)}{I_{n}\left(\omega_{v} R\right)} \\
0 \\
-e_{1}^{T} \mu_{1} q_{n}(0)
\end{array}\right) e_{2}^{T} \phi_{n} e^{i n \theta}\right) A_{n} \\
& +\left(2 \mathcal{B}\left(\mathcal{W}_{n}, W_{0011}\right)+2 \mathcal{B}\left(\mathcal{W}_{-n}, W_{0020}\right)+3 \mathcal{C}\left(\mathcal{W}_{n}, \mathcal{W}_{n}, \mathcal{W}_{-n}\right)\right)\left|A_{n}\right|^{2} A_{n}+\mathcal{O}\left(\overline{A_{n}},{\overline{A_{n}}}^{3}, A_{n}^{3}\right)
\end{aligned}
$$

where $Y$ also satisfies the nontrivial boundary condition

$$
\left[\left.\partial_{r}\left(\begin{array}{c}
D_{u} y_{1} \\
D_{v 0} y_{2}
\end{array}\right)\right|_{r=R}-\left(\begin{array}{c}
K_{u}\left(y_{3}-\left.y_{1}\right|_{r=R}\right) \\
K_{v 0}\left(y_{4}-\left.y_{2}\right|_{r=R}\right)
\end{array}\right)\right]=\left(\begin{array}{c}
0 \\
\beta^{T} \mu_{1} q_{n}(0) e_{2}^{T} \phi_{n}
\end{array}\right) A_{n} e^{i n \theta}+\mathcal{O}\left(\overline{A_{n}}\right) .
$$

Because of their orthogonality property, the contribution from other circular modes will vanish when taking the innerproduct with the adjoint eigenfunction $\mathcal{W}_{n}^{\star}$. Hence, these terms have not been explicitly stated.

The same procedure is applied to the codimension-two case where a pitchfork bifurcation interacts with a Hopf bifurcation. Equating coefficients of $e^{i \lambda_{I} t}$ leads to a linear inhomogeneous equation for the coefficient $X$ given by

$$
\begin{aligned}
& i \lambda_{I} X-\mathcal{L}\left(\mu_{0} ; X\right)=-\mathcal{W}_{0} \frac{d A_{0}}{d \tau}+\left(2 \mathcal{B}\left(\mathcal{W}_{0}, W_{0000}\right)+\left(\begin{array}{c}
0 \\
e_{2}^{T} \mu_{1} \Omega_{v}^{2} B_{0}\left(i \lambda_{I}\right) \frac{I_{0}\left(\Omega_{v} r\right)}{I_{0}\left(\Omega_{v} R\right)} \\
0 \\
-e_{1}^{T} \mu_{1} q_{0}\left(i \lambda_{I}\right)
\end{array}\right) e_{2}^{T} \phi_{0}\right) A_{0} \\
& +\left(2 \mathcal{B}\left(\mathcal{W}_{0}, W_{1100}\right)+2 \mathcal{B}\left(\overline{\mathcal{W}_{0}}, W_{2000}\right)+3 \mathcal{C}\left(\mathcal{W}_{0}, \mathcal{W}_{0}, \overline{\mathcal{W}_{0}}\right)\right)\left|A_{0}\right|^{2} A_{0} \\
& +\left(2 \mathcal{B}\left(\mathcal{W}_{0}, W_{0011}\right)+2 \mathcal{B}\left(\mathcal{W}_{n}, W_{1001}\right)+2 \mathcal{B}\left(\mathcal{W}_{-n}, W_{1010}\right)+6 \mathcal{C}\left(\mathcal{W}_{0}, \mathcal{W}_{n}, \mathcal{W}_{-n}\right)\right)\left|A_{n}\right|^{2} A_{0} \\
& +\mathcal{O}\left(A_{0} A_{n}^{2}, A_{0}{\overline{A_{n}}}^{2}\right)
\end{aligned}
$$

which is subject to the same boundary condition as for the Hopf bifurcation (see equation (2.60)).

The term $Y$, which is constant on the fast time-scale, satisfies

$$
\begin{aligned}
& -\mathcal{L}\left(\mu_{0} ; Y\right)=-\mathcal{W}_{n} \frac{d A_{n}}{d \tau}+\left(2 \mathcal{B}\left(\mathcal{W}_{n}, W_{0000}\right)+\left(\begin{array}{c}
0 \\
e_{2}^{T} \mu_{1} \omega_{v}^{2} B_{n}(0) \frac{I_{n}\left(\omega_{v} r\right)}{I_{n}\left(\omega_{v} R\right)} \\
0 \\
-e_{1}^{T} \mu_{1} q_{n}(0)
\end{array}\right) e_{2}^{T} \phi_{n} e^{i n \theta}\right) A_{n} \\
& +\left(2 \mathcal{B}\left(\mathcal{W}_{n}, W_{0011}\right)+2 \mathcal{B}\left(\mathcal{W}_{-n}, W_{0020}\right)+3 \mathcal{C}\left(\mathcal{W}_{n}, \mathcal{W}_{n}, \mathcal{W}_{-n}\right)\right)\left|A_{n}\right|^{2} A_{n} \\
& +\left(2 \mathcal{B}\left(\mathcal{W}_{n}, W_{1100}\right)+2 \mathcal{B}\left(\mathcal{W}_{0}, W_{0110}\right)+2 \mathcal{B}\left(\overline{\mathcal{W}_{0}}, W_{1010}\right)+6 \mathcal{C}\left(\mathcal{W}_{0}, \overline{\mathcal{W}_{0}}, \mathcal{W}_{n}\right)\right)\left|A_{0}\right|^{2} A_{n} \\
& +\mathcal{O}\left(\overline{A_{n}},{\overline{A_{n}}}^{3}, A_{n}^{3},\left|A_{0}\right|^{2} \overline{A_{n}}\right)
\end{aligned}
$$

and is subject to the same boundary condition as for the pitchfork bifurcation (see equation (2.62)).

The next two lemmas, involving our solvability conditions, provide necessary conditions under which systems (2.59), (2.61), (2.63) and (2.64), together with the appropriate boundary conditions (2.60) and (2.62), admit a solution. Lemma 2.1 is general, while Lemma 2.2 is specific to each bifurcation.

Lemma 2.1. Let $\left\{\mathcal{W}_{n}, \mathcal{W}_{n}^{\star}\right\}$ be an orthogonal pair composed of an eigenfunction and its adjoint, and let $\lambda_{c}$ denote the critical eigenvalue at a given bifurcation point $\mu=\mu_{0} \in \mathbb{R}^{p}$ (p independent bifurcation parameters). Then,

$$
\mathcal{L}\left(\mu_{0} ; \mathcal{W}_{n}\right)=\lambda_{c} \mathcal{W}_{n}, \quad \mathcal{L}^{\star}\left(\mu_{0} ; \mathcal{W}_{n}^{\star}\right)=\overline{\lambda_{c}} \mathcal{W}_{n}^{\star}, \quad \lambda_{c}=\left\{\begin{array}{ll}
i \lambda_{I} & n=0 \\
0 & n \neq 0
\end{array} .\right.
$$

Consider the following linear inhomogeneous system,

$$
\lambda_{c} X-\mathcal{L}\left(\mu_{0} ; X\right)=\mathcal{F}
$$

where $X=\left(x_{1}(r, \theta), x_{2}(r, \theta), x_{3}(\theta), x_{4}(\theta)\right)^{T}$ is subject to the inhomogeneous boundary condition

$$
\left[\left.\partial_{r}\left(\begin{array}{c}
D_{u} x_{1} \\
D_{v} x_{2}
\end{array}\right)\right|_{r=R}-\left(\begin{array}{c}
K_{u}\left(x_{3}-\left.x_{1}\right|_{r=R}\right) \\
K_{v}\left(x_{4}-\left.x_{2}\right|_{r=R}\right)
\end{array}\right)\right]=\left(\begin{array}{c}
\xi(\theta) \\
\eta(\theta)
\end{array}\right) .
$$

Then, a necessary condition for (2.66) to have a solution $X$ subject to (2.67) is that

$$
\left\langle\mathcal{W}_{n}^{\star}, \mathcal{F}\right\rangle+\int_{\partial \Omega} \overline{U_{n}^{\star}} \xi d \sigma+\int_{\partial \Omega} \overline{V_{n}^{\star}} \eta d \sigma=0,
$$

where $U_{n}^{\star}$ and $V_{n}^{\star}$ are the bulk components of the adjoint eigenfunction $\mathcal{W}_{n}^{\star}$. 
Proof. The result follows from a careful application of the Fredholm alternative, where the inhomogeneous boundary condition is taken into account. We take the inner-product with the adjoint eigenfunction on each side of (2.66) to get

$$
\left\langle\mathcal{W}_{n}^{\star}, \lambda_{c} X-\mathcal{L}\left(\mu_{0} ; X\right)\right\rangle=\lambda_{c}\left\langle\mathcal{W}_{n}^{\star}, X\right\rangle-\left\langle\mathcal{W}_{n}^{\star}, \mathcal{L}\left(\mu_{0} ; X\right)\right\rangle=\left\langle\mathcal{W}_{n}^{\star}, \mathcal{F}\right\rangle
$$

Next, by using the definition of the inner-product given in (2.14), one can write

$$
\begin{aligned}
& \left\langle\mathcal{W}_{n}^{\star}, \mathcal{L}\left(\mu_{0} ; X\right)\right\rangle=
\end{aligned}
$$

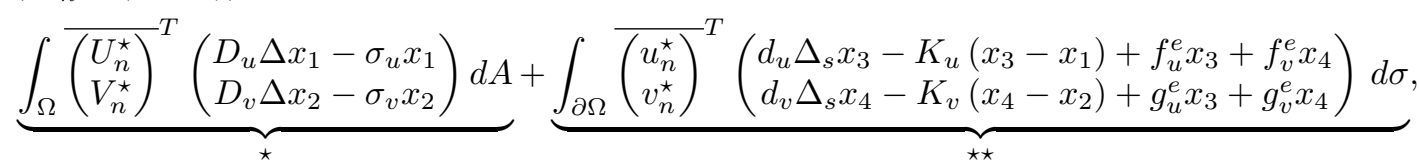

where $\Omega$ is a disk of radius $R$ and $\Delta_{s}=\partial_{\sigma \sigma}$ is the 1-D Laplace-Beltrami operator, with $\sigma=R \theta$ being the arc-length parameterizing the boundary. Next, using Green's second identity, the first integral $(\star)$ can be rewritten as follows:

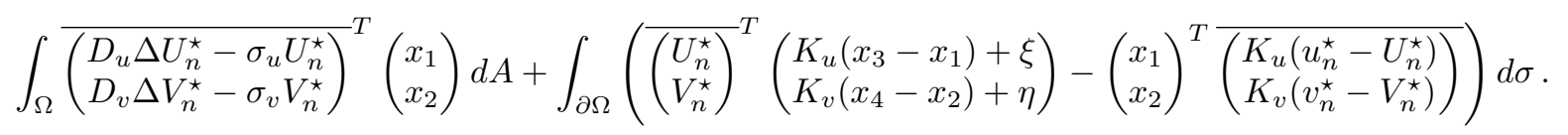

In addition, the second integral can be treated using Lagrange's identity and the angular periodicity of surface bound components, as

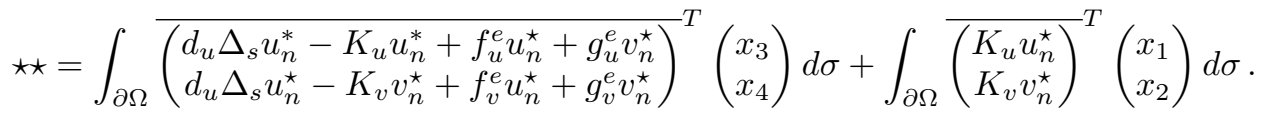

Next, upon adding (2.72) and (2.73), we obtain after some algebra the following expression involving the adjoint linear operator:

$$
\begin{aligned}
\left\langle\mathcal{W}_{n}^{\star}, \mathcal{L}\left(\mu_{0} ; X\right)\right\rangle & =\int_{\Omega} \overline{\left(\begin{array}{c}
D_{u} \Delta U_{n}^{\star}-\sigma_{u} U_{n}^{\star} \\
D_{v} \Delta V_{n}^{\star}-\sigma_{v} V_{n}^{\star}
\end{array}\right)} \text { T }\left(\begin{array}{c}
x_{1} \\
x_{2}
\end{array}\right) d A \\
& +\int_{\partial \Omega} \overline{\left(\begin{array}{c}
d_{u} \Delta_{s} u_{n}^{\star}-K_{u}\left(u_{n}^{\star}-U_{n}^{\star}\right)+f_{u}^{e} u_{n}^{\star}+g_{u}^{e} v_{n}^{\star} \\
d_{u} \Delta_{s} u_{n}^{\star}-K_{v}\left(v_{n}^{\star}-V_{n}^{\star}\right)+f_{v}^{e} u_{n}^{\star}+g_{v}^{e} v_{n}^{\star}
\end{array}\right)}\left(\begin{array}{c}
x_{3} \\
x_{4}
\end{array}\right) d \sigma+\int_{\partial \Omega}\left(\overline{U_{n}^{\star}} \xi+\overline{V_{n}^{\star}} \eta\right) d \sigma \\
& =\left\langle\mathcal{L}^{\star}\left(\mu_{0} ; \mathcal{W}_{n}^{\star}\right), X\right\rangle+\int_{\partial \Omega}\left(\overline{U_{n}^{\star}} \xi+\overline{V_{n}^{\star}} \eta\right) d \sigma \\
& =\lambda_{c}\left\langle\mathcal{W}_{n}^{\star}, X\right\rangle+\int_{\partial \Omega}\left(\overline{U_{n}^{\star}} \xi+\overline{V_{n}^{\star}} \eta\right) d \sigma .
\end{aligned}
$$

The result (2.68) is readily obtained after the substitution of (2.74) back into (2.69).

Lemma 2.2. (Solvability condition). The imposition of the solvability condition for each of the three bifurcations leads to the following amplitude equations:

- Hopf bifurcation. A necessary condition for the inhomogeneous system (2.59) and (2.60) to have a solution $X$ is that the amplitude $A_{0}(\tau)$ satisfies the following ODE:

$$
\begin{aligned}
& -2 \pi R B_{0}\left(i \lambda_{I}\right) q_{0}\left(i \lambda_{I}\right) e_{2}^{T} \overline{\phi_{0}^{\star}} e_{2}^{T} \phi_{0} \beta^{T} \mu_{1} A_{0}=-\frac{d A_{0}}{d \tau}+2\left\langle\mathcal{W}_{0}^{\star}, \mathcal{B}\left(\mathcal{W}_{0}, W_{0000}\right)\right\rangle A_{0} \\
& +2 \pi R e_{2}^{T} \overline{\phi_{0}^{\star}} e_{2}^{T} \phi_{0}\left(\left(B_{0}\left(i \lambda_{I}\right)\right)^{2} \Omega_{v}^{2} \frac{R}{2}\left(1-\left(\frac{I_{1}\left(\Omega_{v} R\right)}{I_{0}\left(\Omega_{v} R\right)}\right)^{2}\right) e_{2}^{T} \mu_{1}-q_{0}\left(i \lambda_{I}\right) e_{1}^{T} \mu_{1}\right) A_{0} \\
& +\left\langle\mathcal{W}_{0}^{\star}, 2 \mathcal{B}\left(\mathcal{W}_{0}, W_{1100}\right)+2 \mathcal{B}\left(\overline{\mathcal{W}_{0}}, W_{2000}\right)+3 \mathcal{C}\left(\mathcal{W}_{0}, \mathcal{W}_{0}, \overline{\mathcal{W}_{0}}\right)\right\rangle\left|A_{0}\right|^{2} A_{0} .
\end{aligned}
$$

- Pitchfork bifurcation. A necessary condition for the inhomogeneous system (2.61) and (2.62) to have a solution $Y$ is that the amplitude $A_{n}(\tau)$ satisfies the following $O D E$ :

$$
\begin{aligned}
& -2 \pi R B_{n}(0) q_{n}(0) e_{2}^{T} \boldsymbol{\phi}_{n}^{\star} e_{2}^{T} \boldsymbol{\phi}_{n} \beta^{T} \mu_{1} A_{n}=-\frac{d A_{n}}{d \tau}+2\left\langle\mathcal{W}_{n}^{\star}, \mathcal{B}\left(\mathcal{W}_{n}, W_{0000}\right)\right\rangle A_{n} \\
& +2 \pi R e_{2}^{T} \boldsymbol{\phi}_{n}^{\star} e_{2}^{T} \boldsymbol{\phi}_{n}\left(\left(B_{n}(0)\right)^{2} \omega_{v}^{2} \frac{R}{2}\left(1-\frac{I_{n-1}\left(\omega_{v} R\right) I_{n+1}\left(\omega_{v} R\right)}{\left(I_{n}\left(\omega_{v} R\right)\right)^{2}}\right) e_{2}^{T} \mu_{1}-q_{n}(0) e_{1}^{T} \mu_{1}\right) A_{n} \\
& +\left\langle\mathcal{W}_{n}^{\star}, 2 \mathcal{B}\left(\mathcal{W}_{n}, W_{0011}\right)+2 \mathcal{B}\left(\mathcal{W}_{-n}, W_{0020}\right)+3 \mathcal{C}\left(\mathcal{W}_{n}, \mathcal{W}_{n}, \mathcal{W}_{-n}\right)\right\rangle\left|A_{n}\right|^{2} A_{n} .
\end{aligned}
$$

- Pitchfork-Hopf bifurcation. A necessary condition for the inhomogeneous system (2.59), (2.61), (2.60), and (2.62) to have solutions $X$ and $Y$ is that the amplitudes $A_{0}(\tau)$ and $A_{n}(\tau)$ satisfy the following system of ODEs:

$$
-2 \pi R B_{0}\left(i \lambda_{I}\right) q_{0}\left(i \lambda_{I}\right) e_{2}^{T} \overline{\phi_{0}^{\star}} e_{2}^{T} \phi_{0} \beta^{T} \mu_{1} A_{0}=-\frac{d A_{0}}{d \tau}+2\left\langle\mathcal{W}_{0}^{\star}, \mathcal{B}\left(\mathcal{W}_{0}, W_{0000}\right)\right\rangle A_{0}
$$




$$
\begin{aligned}
& +2 \pi R e_{2}^{T} \overline{\phi_{0}^{\star}} e_{2}^{T} \phi_{0}\left(\left(B_{0}\left(i \lambda_{I}\right)\right)^{2} \Omega_{v}^{2} \frac{R}{2}\left(1-\left(\frac{I_{1}\left(\Omega_{v} R\right)}{I_{0}\left(\Omega_{v} R\right)}\right)^{2}\right) e_{2}^{T} \mu_{1}-q_{0}\left(i \lambda_{I}\right) e_{1}^{T} \mu_{1}\right) A_{0} \\
& +\left\langle\mathcal{W}_{0}^{\star}, 2 \mathcal{B}\left(\mathcal{W}_{0}, W_{1100}\right)+2 \mathcal{B}\left(\overline{\mathcal{W}_{0}}, W_{2000}\right)+3 \mathcal{C}\left(\mathcal{W}_{0}, \mathcal{W}_{0}, \overline{\mathcal{W}_{0}}\right)\right\rangle\left|A_{0}\right|^{2} A_{0} \\
& +\left\langle\mathcal{W}_{0}^{\star}, 2 \mathcal{B}\left(\mathcal{W}_{0}, W_{0011}\right)+2 \mathcal{B}\left(\mathcal{W}_{n}, W_{1001}\right)+2 \mathcal{B}\left(\mathcal{W}_{-n}, \mathcal{W}_{1010}\right)+6 \mathcal{C}\left(\mathcal{W}_{0}, \mathcal{W}_{n}, \mathcal{W}_{-n}\right)\right\rangle\left|A_{n}\right|^{2} A_{0}
\end{aligned}
$$

and

$$
\begin{aligned}
& -2 \pi R B_{n}(0) q_{n}(0) e_{2}^{T} \phi_{n}^{\star} e_{2}^{T} \phi_{n} \beta^{T} \mu_{1} A_{n}=-\frac{d A_{n}}{d \tau}+2\left\langle\mathcal{W}_{n}^{\star}, \mathcal{B}\left(\mathcal{W}_{n}, W_{0000}\right)\right\rangle A_{n} \\
& +2 \pi R e_{2}^{T} \phi_{n}^{\star} e_{2}^{T} \phi_{n}\left(\left(B_{n}(0)\right)^{2} \omega_{v}^{2} \frac{R}{2}\left(1-\frac{I_{n-1}\left(\omega_{v} R\right) I_{n+1}\left(\omega_{v} R\right)}{\left(I_{n}\left(\omega_{v} R\right)\right)^{2}}\right) e_{2}^{T} \mu_{1}-q_{n}(0) e_{1}^{T} \mu_{1}\right) A_{n} \\
& +\left\langle\mathcal{W}_{n}^{\star}, 2 \mathcal{B}\left(\mathcal{W}_{n}, W_{0011}\right)+2 \mathcal{B}\left(\mathcal{W}_{-n}, W_{0020}\right)+3 \mathcal{C}\left(\mathcal{W}_{n}, \mathcal{W}_{n}, \mathcal{W}_{-n}\right)\right\rangle\left|A_{n}\right|^{2} A_{n} \\
& +\left\langle\mathcal{W}_{n}^{\star}, 2 \mathcal{B}\left(\mathcal{W}_{n}, W_{1100}\right)+2 \mathcal{B}\left(\mathcal{W}_{0}, W_{0110}\right)+2 \mathcal{B}\left(\overline{\mathcal{W}_{0}}, W_{1010}\right)+6 \mathcal{C}\left(\mathcal{W}_{0}, \overline{\mathcal{W}_{0}}, \mathcal{W}_{n}\right)\right\rangle\left|A_{0}\right|^{2} A_{n}
\end{aligned}
$$

In each of these three cases, $\mathcal{W}_{0}^{\star}$ and $\mathcal{W}_{n}^{\star}$ are adjoint eigenfunctions associated with the adjoint linear operator. Hence, they satisfy the following relations:

$$
\mathcal{L}^{\star}\left(\mu_{0}, \mathcal{W}_{0}^{\star}\right)=-i \lambda_{I} \mathcal{W}_{0}^{\star}, \quad \mathcal{L}^{\star}\left(\mu_{0}, \mathcal{W}_{n}^{\star}\right)=0
$$

Proof. The results stated above are obtained from a direct application of Lemma 2.1.

The rearrangement of each of the differential equations given in Lemma 2.2 provides a system of amplitude equations describing the branching behavior in the vicinity of the bifurcation point, in the limit $\varepsilon \rightarrow 0$. For each of the three bifurcations the amplitude equations have the following form:

\section{- Hopf bifurcation.}

$$
\frac{d A_{0}}{d \tau}=g_{1000}^{T} \mu_{1} A_{0}+g_{2100}\left|A_{0}\right|^{2} A_{0}
$$

\section{- Pitchfork bifurcation.}

$$
\frac{d A_{n}}{d \tau}=g_{0010}^{T} \mu_{1} A_{n}+g_{0021}\left|A_{n}\right|^{2} A_{n}
$$

- Pitchfork-Hopf bifurcation. In this case, one shall analyze a system of two amplitude equations similar to their codimension-one analog. In each equation, the additional term corresponds to the mixed-mode term (all the other terms remain the same as for their codimension-one counterpart).

$$
\begin{aligned}
& \frac{d A_{0}}{d \tau}=g_{1000}^{T} \mu_{1} A_{0}+g_{2100}\left|A_{0}\right|^{2} A_{0}+g_{1011}\left|A_{n}\right|^{2} A_{0} \\
& \frac{d A_{n}}{d \tau}=g_{0010}^{T} \mu_{1} A_{n}+g_{0021}\left|A_{n}\right|^{2} A_{n}+g_{1110}\left|A_{0}\right|^{2} A_{n} .
\end{aligned}
$$

Explicit expressions for the coefficients of the nonlinear terms in the amplitude equations are

$$
\begin{aligned}
& g_{2100}=\left\langle\mathcal{W}_{0}^{\star}, 2 \mathcal{B}\left(\mathcal{W}_{0}, W_{1100}\right)+2 \mathcal{B}\left(\overline{\mathcal{W}_{0}}, W_{2000}\right)+3 \mathcal{C}\left(\mathcal{W}_{0}, \mathcal{W}_{0}, \overline{\mathcal{W}_{0}}\right)\right\rangle \\
& g_{1011}=\left\langle\mathcal{W}_{0}^{\star}, 2 \mathcal{B}\left(\mathcal{W}_{0}, W_{0011}\right)+2 \mathcal{B}\left(\mathcal{W}_{n}, W_{1001}\right)+2 \mathcal{B}\left(\mathcal{W}_{-n}, \mathcal{W}_{1010}\right)+6 \mathcal{C}\left(\mathcal{W}_{0}, \mathcal{W}_{n}, \mathcal{W}_{-n}\right)\right\rangle \\
& g_{0021}=\left\langle\mathcal{W}_{n}^{\star}, 2 \mathcal{B}\left(\mathcal{W}_{n}, W_{0011}\right)+2 \mathcal{B}\left(\mathcal{W}_{-n}, W_{0020}\right)+3 \mathcal{C}\left(\mathcal{W}_{n}, \mathcal{W}_{n}, \mathcal{W}_{-n}\right)\right\rangle \\
& g_{1110}=\left\langle\mathcal{W}_{n}^{\star}, 2 \mathcal{B}\left(\mathcal{W}_{n}, W_{1100}\right)+2 \mathcal{B}\left(\mathcal{W}_{0}, W_{0110}\right)+2 \mathcal{B}\left(\overline{\mathcal{W}_{0}}, W_{1010}\right)+6 \mathcal{C}\left(\mathcal{W}_{0}, \overline{\mathcal{W}_{0}}, \mathcal{W}_{n}\right)\right\rangle
\end{aligned}
$$

where $g_{2100}, g_{1011} \in \mathbb{C}$ and $g_{0021}, g_{1110} \in \mathbb{R}$. The coefficients of the linear terms consist of the projection of the vectors $g_{1000} \in \mathbb{C}^{2}$ and $g_{0010} \in \mathbb{R}^{2}$ onto the detuning unit vector $\mu_{1}$. They are given by

$$
\begin{aligned}
g_{1000} & =2 \pi R e_{2}^{T} \overline{\boldsymbol{\phi}_{0}^{\star}} e_{2}^{T} \boldsymbol{\phi}_{0}\left(B_{0}\left(i \lambda_{I}\right) q_{0}\left(i \lambda_{I}\right) \beta+\left(B_{0}\left(i \lambda_{I}\right)\right)^{2} \Omega_{v}^{2} \frac{R}{2}\left(1-\left(\frac{I_{1}\left(\Omega_{v} R\right)}{I_{0}\left(\Omega_{v} R\right)}\right)^{2}\right) e_{2}-q_{0}\left(i \lambda_{I}\right) e_{1}\right) \\
& +4 \pi R{\overline{\phi_{0}^{\star}}}^{T} B\left(\phi_{0}, \tilde{\boldsymbol{u}}_{0000}\right) \alpha \\
g_{0010} & =2 \pi R e_{2}^{T} \boldsymbol{\phi}_{n}^{\star} e_{2}^{T} \boldsymbol{\phi}_{n}\left(B_{n}(0) q_{n}(0) \beta+\left(B_{n}(0)\right)^{2} \omega_{v}^{2} \frac{R}{2}\left(1-\frac{I_{n-1}\left(\omega_{v} R\right) I_{n+1}\left(\omega_{v} R\right)}{\left(I_{n}\left(\omega_{v} R\right)\right)^{2}}\right) e_{2}-q_{n}(0) e_{1}\right) \\
& +4 \pi R \phi_{n}^{\star T} B\left(\phi_{n}, \tilde{\boldsymbol{u}}_{0000}\right) \alpha
\end{aligned}
$$

where $\tilde{\boldsymbol{u}}_{0000}=\left[\boldsymbol{\Phi}_{0}\left(\mu_{0} ; 0\right)\right]^{-1} E_{2} \boldsymbol{u}_{e}$ appears in the expression for $\boldsymbol{u}_{0000}$ (see equation (2.42)). The two quantities are related to each other by

$$
\boldsymbol{u}_{0000}=\tilde{\boldsymbol{u}}_{0000} \alpha^{T} \mu_{1} .
$$

In $\S 4$ below, the solution behaviors in the weakly nonlinear regime, as predicted after the numerical evaluation of the coefficients in the amplitude equations, will be compared with numerical PDE simulations of the full coupled bulk-surface reaction-diffusion model. 
We remark that the generic form for the coefficients of the cubic terms of $(2.80)$ - (2.82) is well-known in the literature. Since the pitchfork bifurcation is simply a Hopf bifurcation with a zero-crossing eigenvalue, these coefficients also arise from the weakly nonlinear analysis of a codimension-two Hopf-Hopf bifurcation, in which the pair of critical eigenfunctions is fully complex. We refer the reader to the appendix of [11], where a center manifold reduction was used to derive a two-dimensional system of amplitude equations at a double-Hopf bifurcation.

We have performed a systematic derivation yielding explicit formulae for the normal form coefficients for the three distinct bifurcations in our coupled bulk-surface model. This involved a careful analysis of the underlying spectral problem. These normal form results can be used for any nonlinear surface reaction kinetics specified on the circular boundary. The bifurcation parameters employed here, being the bulk diffusion coefficient $D_{v}$ and the coupling rate constant $K_{v}$, are used below to understand the specific role of bulk-surface coupling. In our derivation above we have allowed for an arbitrary sweep of the two-parameter vector $\left(K_{v}, D_{v}\right)$ across linear stability boundaries. Our choice of performing an asymptotic multiple time-scale analysis, as opposed to a center manifold reduction, was partially motivated by computational convenience and flexibility. The main advantage of multi-scale theory concerns the ease with which bifurcation parameters can be treated if they appear in the boundary conditions (1.3). By simply keeping track of the terms at each order within the boundary conditions, the method of multiple time-scales provides a direct approach to compute the normal form.

\section{Bifurcation analysis of amplitude equations}

In this section, the equilibria of the amplitude equations derived in the preceding section are analyzed. Under nondegeneracy conditions, the stability properties of the steady states in the normal form are preserved in the full model. Bifurcations of codimension-one and two are treated separately below.

\subsection{Codimension-one: Hopf and pitchfork bifurcations}

For a Hopf bifurcation, the substitution of $A_{0}(\tau)=\rho_{0}(\tau) e^{i \theta_{0}(\tau)}$ within (2.80) yields a coupled system of ODEs for the magnitude $\rho_{0}$ and the phase $\theta_{0}$, given by

$$
\frac{d \rho_{0}}{d \tau}=\left[\Re\left(g_{1000}\right)\right]^{T} \mu_{1} \rho_{0}+\Re\left(g_{2100}\right) \rho_{0}^{3}, \quad \frac{d \theta_{0}}{d \tau}=\left[\Im\left(g_{1000}\right)\right]^{T} \mu_{1}+\Im\left(g_{2100}\right) \rho_{0}^{2} .
$$

When it exists, a steady state $\rho_{0 e}$ of (3.1) is given by

$$
\rho_{0 e}=\sqrt{-\frac{\left[\Re\left(g_{1000}\right)\right]^{T} \mu_{1}}{\Re\left(g_{2100}\right)}},
$$

where $\mu_{1}$ is a free two-dimensional unit vector. The transversality condition is violated when $\mu_{1}$ is tangent to the Hopf stability boundary at $\mu_{0} \in \mathbb{R}^{2}$, for which $\rho_{0 e}$ from (3.2) vanishes. Consequently, it can easily be argued that the vector $\Re\left(g_{1000}\right)$ is normal to the stability curve and that a natural choice for the orientation of $\mu_{1}$ is

$$
\mu_{1}=-\frac{\Re\left(g_{2100}\right)}{\left|\Re\left(g_{2100}\right)\right|} \frac{\Re\left(g_{1000}\right)}{\left\|\Re\left(g_{1000}\right)\right\|} .
$$

Such a choice provides the maximal magnitude $\rho_{0 e}$ given by

$$
\rho_{0 e}=\sqrt{\frac{\left\|\Re\left(g_{1000}\right)\right\|}{\left|\Re\left(g_{2100}\right)\right|}} .
$$

The nontrivial fixed points $\rho_{0 e}$ in (3.2) correspond to limit cycle of the complex amplitude $A_{0}(\tau)$. The stability of the limit cycles is given by the sign of the real part of the cubic term coefficient in (3.1). Hence, one can distinguish between supercritical and subcritical bifurcations in the usual way:

$$
\Re\left(g_{2100}\right)<0 \text { (Supercritical Hopf) }, \quad \Re\left(g_{2100}\right)>0 \text { (Subcritical Hopf) . }
$$

Next, from substituting $\rho_{0 e}$ into the equation for the phase in (3.1), the steady state phase $\theta_{0}$ is

$$
\theta_{0}(\tau)=\theta_{0}(0)+\tilde{\theta}_{0} \tau, \quad \text { with } \quad \tilde{\theta}_{0}=\left[\Im\left(g_{1000}\right)\right]^{T} \mu_{1}+\Im\left(g_{2100}\right) \rho_{0 e}^{2},
$$

where $\theta_{0}(0) \in \mathbb{R}$ denotes the phase shift symmetry of periodic solutions.

The following lemma uses the nontrivial steady state from the amplitude equation associated with the Hopf bifurcation to give approximate periodic solutions of the full coupled bulk-surface PDE model in the weakly nonlinear regime:

Lemma 3.1. (Periodic solutions in the weakly nonlinear regime). Let $g_{2100} \in \mathbb{C}$ be the cubic term coefficient in (2.80), and assume that its real part is nonzero, hence excluding degenerate cases. Then, in the limit $\varepsilon \rightarrow 0$ with $\varepsilon=\sqrt{\left\|\mu-\mu_{0}\right\|}$ denoting the square-root of the distance with the Hopf bifurcation point, a leading-order approximate family of periodic solutions is given by

$$
W(t)=W_{e}+\varepsilon \rho_{0 e}\left[\mathcal{W}_{0} e^{i\left(\lambda_{I} t+\theta_{0}(0)\right)}+\overline{\mathcal{W}_{0}} e^{-i\left(\lambda_{I} t+\theta_{0}(0)\right)}\right]+\mathcal{O}\left(\varepsilon^{2}\right)
$$


for any $\theta_{0}(0) \in \mathbb{R}$ and with $\rho_{0 e}$ defined by (3.4).

For the surface-bound activator species, let $u_{a m p}$ denote the amplitude of the bifurcating limit cycle near the Hopf bifurcation point. A leading-order approximation for $u_{a m p}$ is

$$
u_{a m p}=\max _{0 \leq t<T_{p}}\left\{\left|u(t)-u_{e}\right|\right\}=2 \varepsilon \rho_{0 e}\left|e_{1}^{T} \phi_{0}\right|+\mathcal{O}\left(\varepsilon^{2}\right)
$$

where the period $T_{p}$ satisfies

$$
T_{p}=\frac{2 \pi}{\lambda_{I}}+\mathcal{O}\left(\varepsilon^{2}\right)
$$

Finally, the periodic solution in (3.7) is asymptotically stable when $\Re\left(g_{2100}\right)<0$ (supercritical Hopf) and it is unstable for $\Re\left(g_{2100}\right)>0$ (subcritical Hopf).

Next, we consider the pitchfork bifurcation. Since the coefficients $g_{0010}$ and $g_{0021}$ in $(2.81)$ are real, we set $A_{n}(\tau)=$ $\rho_{n}(\tau) e^{i \theta_{n}(\tau)}$ into (2.81) to obtain a decoupled system of ODEs for $\rho_{n}$ and $\theta_{n}$ given by

$$
\frac{d \rho_{n}}{d \tau}=g_{0010}^{T} \mu_{1} \rho_{n}+g_{0021} \rho_{n}^{3}, \quad \frac{d \theta_{n}}{d \tau}=0 .
$$

When they exist, fixed points of (3.10) are

$$
\rho_{n e}=\sqrt{-\frac{g_{0010}^{T} \mu_{1}}{g_{0021}}}, \quad \theta_{n}(\tau) \equiv \theta_{n} \in \mathbb{R},
$$

where the constant $\theta_{n}$ accounts for the rotational equivariance of the pattern. The orientation of the unit vector $\mu_{1}$ is chosen in a similar way as for the Hopf bifurcation, with the "natural choice" being

$$
\mu_{1}=-\frac{g_{0021}}{\left|g_{0021}\right|} \frac{g_{0010}}{\left\|g_{0010}\right\|}
$$

This yields the following expression for $\rho_{n e}$ :

$$
\rho_{n e}=\sqrt{\frac{\left\|g_{0010}\right\|}{\left|g_{0021}\right|}} .
$$

Again, the pitchfork bifurcation is classified according to the stability of the nontrivial steady state of the amplitude equation. It readily follows that

$$
g_{0021}<0 \text { (Supercritical pitchfork), } \quad g_{0021}>0 \text { (Subcritical pitchfork) }
$$

Finally, this section is concluded with the analog of Lemma 3.1 for Turing-type patterns arising from pitchfork bifurcations in the full coupled bulk-surface PDE model.

Lemma 3.2. (Spatially inhomogeneous equilibria in the weakly nonlinear regime). Let $g_{0021} \in \mathbb{R}$ be the cubic term coefficient in (2.81), and assume that it is nonzero, hence excluding degenerate cases. Then, in the limit $\varepsilon \rightarrow 0$ with $\varepsilon=\sqrt{\left\|\mu-\mu_{0}\right\|}$ denoting the square-root of the distance from the pitchfork bifurcation point, a leading-order approximate family of spatially inhomogeneous equilibria is given by

$$
W=W_{e}+\varepsilon \rho_{n e}\left[\mathcal{W}_{n} e^{i \theta_{n}}+\mathcal{W}_{-n} e^{-i \theta_{n}}\right]+\mathcal{O}\left(\varepsilon^{2}\right),
$$

for any $\theta_{n} \in \mathbb{R}$ and with $\rho_{\text {ne }}$ defined by (3.13).

For the surface-bound activator species, let $u_{a m p}$ be the amplitude of the bifurcating Turing-type pattern near the pitchfork bifurcation point. A leading-order approximation for $u_{a m p}$ is

$$
u_{a m p}=\max _{0 \leq \theta<2 \pi}\left\{\left|u(\theta)-u_{e}\right|\right\}=2 \varepsilon \rho_{n e}\left|e_{1}^{T} \phi_{n}\right|+\mathcal{O}\left(\varepsilon^{2}\right)
$$

Finally, the patterned solution given by (3.15) is asymptotically stable when $g_{0021}<0$ (supercritical pitchfork), and it is unstable for $g_{0021}>0$ (subcritical pitchfork).

\subsection{Codimension-two: Pitchfork-Hopf bifurcation}

In this subsection, the equilibria of the coupled amplitude equations (2.82) are analyzed for general values of its coefficients. For this purpose, it is appropriate to rescale the time variable into the original fast time-scale $t$. Hence, letting $z_{0}(t)=$ $\varepsilon A_{0}\left(\varepsilon^{2} t\right) e^{i \lambda_{I} t}$ and $z_{n}(t)=\varepsilon A_{n}\left(\varepsilon^{2} t\right)$, and recalling that $\mu-\mu_{0}=\varepsilon^{2} \mu_{1}$, the following Poincaré normal form can readily be obtained:

$$
\begin{aligned}
& \dot{z}_{0}=\left(i \lambda_{I}+g_{1000}^{T}\left(\mu-\mu_{0}\right)\right) z_{0}+g_{2100}\left|z_{0}\right|^{2} z_{0}+g_{1011}\left|z_{n}\right|^{2} z_{0} \\
& \dot{z}_{n}=g_{0010}^{T}\left(\mu-\mu_{0}\right) z_{n}+g_{1110}\left|z_{0}\right|^{2} z_{n}+g_{0021}\left|z_{n}\right|^{2} z_{n} .
\end{aligned}
$$


Since $g_{2100}, g_{1011}$ are complex-valued, while $g_{0021}, g_{1110}$ are real-valued, cylindrical polar coordinates are appropriate for this normal form. Letting $z_{0}=r e^{i \phi}, z_{n}=w \geq 0$, and $\mu=\mu_{0}$ we obtain a system of ODEs for the magnitudes $w, r$ and the phase $\phi$, given by

$$
\dot{w}=g_{0021} w^{3}+g_{1110} w r^{2}, \quad \dot{r}=\Re\left(g_{1011}\right) w^{2} r+\Re\left(g_{0021}\right) r^{3}, \quad \dot{\phi}=\lambda_{I}+\Im\left(g_{2100}\right) r^{2}+\Im\left(g_{1011}\right) w^{2} .
$$

Since the third equation above is decoupled from the others, it need not be considered. The first two ODEs in (3.18) can then be conveniently written as

$$
\dot{w}=p_{11} w^{3}+p_{12} w r^{2}, \quad \dot{r}=p_{21} w^{2} r+p_{22} r^{3},
$$

where $p_{11}=g_{0021}, p_{12}=g_{1110}, p_{21}=\Re\left(g_{1011}\right)$ and $p_{22}=\Re\left(g_{2100}\right)$. It is assumed that these coefficients are nonzero and that $p_{11} p_{22}-p_{12} p_{21} \neq 0$, which is a necessary condition for the existence of the mixed-mode steady state.

To relate our results with those in [13], we augment (3.19) with linear terms to obtain

$$
\dot{w}=w\left(\delta_{1}+p_{11} w^{2}+p_{12} r^{2}\right), \quad \dot{r}=r\left(\delta_{2}+p_{21} w^{2}+p_{22} r^{2}\right),
$$

where $\delta_{1}$ and $\delta_{2}$ are generic unfolding parameters. This recovers the well-known canonical truncated system of amplitude equations at a codimension-two pitchfork-Hopf and double-Hopf bifurcations. We refer the reader to [13] and [17], where the phase portraits of (3.20) is classified. In the discussion below, the approach and classification from [13] is followed.

We first reduce the number of parameters in (3.20) by setting $\bar{w}=\sqrt{\left|p_{11}\right|} w$ and $\bar{r}=\sqrt{\left|p_{22}\right|}$. After a possible time rescaling if $p_{11}<0$, and further dropping the bars to simplify the notation, this change of variable yields

$$
\dot{w}=w\left(\delta_{1}+w^{2}+\gamma r^{2}\right), \quad \dot{r}=r\left(\delta_{2}+\eta w^{2}+d r^{2}\right),
$$

where $\gamma, \eta$ and $d$ are given by

$$
\gamma=\frac{p_{12}}{\left|p_{22}\right|}, \quad \eta=\frac{p_{21}}{\left|p_{11}\right|}, \quad d=\frac{p_{22}}{\left|p_{22}\right|}= \pm 1
$$

Depending on the signs of the four quantities $d, \gamma, \eta$ and $d-\gamma \eta$, it is possible to distinguish between 12 topologically different stability diagrams in the plane of generic parameters $\left(\delta_{1}, \delta_{2}\right)$ (see [13]). In addition to the trivial equilibrium $E_{0}=(0,0),(3.21)$ possesses up to three additional steady states. Two of those equilibria are located on the coordinate axes and are given by

$$
E_{1}=\left(\sqrt{-\delta_{1}}, 0\right) \quad \text { for } \delta_{1}<0, \quad \text { and } \quad E_{2}=\left(0, \sqrt{-\frac{\delta_{2}}{d}}\right) \quad \text { for } \frac{\delta_{2}}{d}<0
$$

while the last one corresponds to the mixed-mode equilibrium defined by

$$
E_{3}=\left(\sqrt{\frac{\gamma \delta_{2}-d \delta_{1}}{d-\gamma \eta}}, \sqrt{\frac{\eta \delta_{1}-\delta_{2}}{d-\gamma \eta}}\right) \quad \text { for } \quad \frac{\gamma \delta_{2}-d \delta_{1}}{d-\gamma \eta}, \frac{\eta \delta_{1}-\delta_{2}}{d-\gamma \eta}>0 .
$$

With regards to the coupled bulk-surface model, these equilibria have a precise meaning in terms of oscillatory and patterned solutions. They are classified below in Table 1.

Table 1: Classification of steady states of (3.21) and solution correspondence.

\begin{tabular}{|c|c|}
\hline Amplitude solution & Full model solution \\
\hline Trivial steady state $E_{0}$ & Base-state solution $W_{e}$ \\
\hline First mode $E_{1}$ & Spatially inhomogeneous steady state \\
\hline Second mode $E_{2}$ & Periodic solution around the base state solution \\
\hline Mixed-mode $E_{3}$ & Oscillations around a patterned solution (breather solution) \\
\hline
\end{tabular}

We now summarize the bifurcations arising in the truncated system of amplitude equations (3.21). Firstly, the single mode equilibria $E_{1}$ and $E_{2}$ bifurcate from the origin on the lines

$$
H_{1}=\left\{\left(\delta_{1}, \delta_{2}\right) \mid \delta_{1}=0\right\}, \quad H_{2}=\left\{\left(\delta_{1}, \delta_{2}\right) \mid \delta_{2}=0\right\},
$$

and, respectively, exist for $\delta_{1}<0$ and for $\delta_{2} / d<0$. Next, the mixed-mode equilibrium $E_{3}$ bifurcates from each of the single mode equilibria on the semi-infinite pitchfork lines, given by

$$
\begin{aligned}
& T_{1}=\left\{\left(\delta_{1}, \delta_{2}\right) \mid \gamma \delta_{2}=d \delta_{1}, \quad \delta_{1}>0 \text { or } \delta_{1}<0\right\} \\
& T_{2}=\left\{\left(\delta_{1}, \delta_{2}\right) \mid \delta_{2}=\eta \delta_{1}, \delta_{1}>0 \text { or } \delta_{1}<0\right\}
\end{aligned}
$$

whose orientations (whether for each case $\delta_{1}$ is positive or negative) are chosen such that $T_{1}$ and $T_{2}$ form the boundaries of the existence region of $E_{3}$.

Cases for which $d=1$ are known to be simple since the truncated system of amplitude equations (3.21) does not possess any limit cycles. Such a situation corresponds to both pitchfork and Hopf bifurcations being either subcritical 
or supercritical, with no fifth-order terms being needed in the normal form. More intricate cases arise when $d=-1$ and $d-\gamma \eta>0$, at which $E_{3}$ bifurcates through a degenerate Hopf bifurcation on the semi-infinite line given by

$$
C=\left\{\left(\delta_{1}, \delta_{2}\right) \mid \delta_{2}=\frac{d(1-\eta)}{\gamma-d} \delta_{1}, \quad \delta_{1}>0 \text { or } \delta_{1}<0\right\} .
$$

The stability of the bifurcating limit cycle is typically determined by including one fifth-order term to the normal form. More details on this challenging computation are found in the classic reference [13].

In the next lemma, an affine transformation mapping parameter spaces is defined.

Lemma 3.3. (Mapping parameter spaces). Let $\mathcal{T}$ be an affine transformation in $\mathbb{R}^{2}$ that maps the generic parameter space defined by $\delta_{1}$ and $\delta_{2}$ to the original bifurcation parameter space defined by $K_{v}$ and $D_{v}$. Then, it must satisfy

$$
\begin{aligned}
\mathcal{T}\left(\delta_{1}, \delta_{2}\right) & =\mu_{0}+\mathcal{R}\left( \pm \frac{\pi}{2}\right)\left[\begin{array}{ll}
g_{0010} & \Re\left(g_{1000}\right)
\end{array}\right] \mathcal{R}\left( \pm \frac{\pi}{2}\right)\left(\begin{array}{l}
\delta_{1} \\
\delta_{2}
\end{array}\right) \\
& =\mu_{0}+\left[\begin{array}{ll}
\mathcal{R}\left(\frac{\pi}{2}\right) \Re\left(g_{1000}\right) & \mathcal{R}\left(-\frac{\pi}{2}\right) g_{0010}
\end{array}\right]\left(\begin{array}{l}
\delta_{1} \\
\delta_{2}
\end{array}\right)
\end{aligned}
$$

where $\mu_{0}=\left(K_{v 0}, D_{v 0}\right)^{T}$ is the codimension-two bifurcation point and $\mathcal{R}(\varphi)$ is the anti-clockwise rotation matrix in the Euclidean plane defined by

$$
\mathcal{R}(\varphi)=\left(\begin{array}{cc}
\cos (\varphi) & -\sin (\varphi) \\
\sin (\varphi) & \cos (\varphi)
\end{array}\right)
$$

Proof. The proof follows from the fact that $g_{0010}$ and $\Re\left(g_{1000}\right)$ are, respectively, normal to the pitchfork $n=1$ and Hopf $n=0$ stability boundaries.

This subsection concludes with Lemma 3.4, where the mixed-mode solution in the weakly nonlinear regime is defined. Its stability will be discussed in $\S 4.2$ for two pitchfork-Hopf bifurcations involving distinct reaction kinetics.

Lemma 3.4. (Mixed-mode solution in the weakly nonlinear regime). Let $p_{11}=g_{0021}, p_{12}=g_{1110}, p_{21}=\Re\left(g_{1011}\right)$ and $p_{22}=\Re\left(g_{2100}\right)$ be the four cubic term coefficients of system (2.82). If none of these coefficients vanish, and if $p_{22} p_{11}-p_{12} p_{21}$ is nonzero, then in the limit $\varepsilon \rightarrow 0$, with $\varepsilon=\sqrt{\left\|\mu-\mu_{0}\right\|}$ denoting the square-root of the distance with the pitchfork-Hopf bifurcation point, a leading-order approximate family of spatio-temporal oscillatory solutions is given by

$$
W(t)=W_{e}+\varepsilon\left(\rho_{n e}\left[\mathcal{W}_{n} e^{i \theta_{n}}+\text { c.c. }\right]+\rho_{0 e}\left[\mathcal{W}_{0} e^{i\left(\lambda_{I} t+\theta_{0}(0)\right)}+\text { c.c. }\right]\right)+\mathcal{O}\left(\varepsilon^{2}\right)
$$

for any $\theta_{n}, \theta_{0}(0) \in \mathbb{R}$, as a result of the azimuthal and temporal phase shift symmetries. The pair $\left(\rho_{n e}, \rho_{0 e}\right)$ corresponds to the mixed-mode equilibrium and is given by

$$
\left(\rho_{n e}, \rho_{0 e}\right)=\left(\sqrt{\frac{p_{12}\left[\Re\left(g_{1000}\right)\right]^{T} \mu_{1}-p_{22}\left[g_{0010}\right]^{T} \mu_{1}}{p_{22} p_{11}-p_{12} p_{21}}}, \sqrt{\frac{p_{21}\left[g_{0010}\right]^{T} \mu_{1}-p_{11}\left[\Re\left(g_{1000}\right)\right]^{T} \mu_{1}}{p_{22} p_{11}-p_{12} p_{21}}}\right),
$$

with the detuning vector $\mu_{1}$ chosen such that $\mu=\mu_{0}+\varepsilon^{2} \mu_{1}$ is within the mixed-mode region of existence. In particular, it can be taken to be parallel to the bisector of this region.

For the surface-bound activator species, let $u_{a m p}$ be the amplitude of the bifurcating spatio-temporal pattern near the pitchfork-Hopf bifurcation point. A leading-order approximation for it is given by

$$
u_{a m p}=\max _{\substack{0 \leq \theta<2 \pi \\ 0 \leq t<T_{p}}}\left\{\left|u(\theta, t)-u_{e}\right|\right\}=2 \varepsilon\left(\rho_{n e}\left|e_{1}^{T} \phi_{n}\right|+\rho_{0 e}\left|e_{1}^{T} \phi_{0}\right|\right)+\mathcal{O}\left(\varepsilon^{2}\right) .
$$

\section{Validation of weakly nonlinear theory}

In this section we validate the weakly nonlinear theory developed in $\S 2$ and $\S 3$ by comparing predictions of this theory with either numerical bifurcation results or full numerical time-dependent PDE solutions. In the comparisons we will consider both the Schnakenberg (1.5) and Brusselator (1.6) boundary reaction kinetics. Since the parameter space is large, the bulk domain is restricted to the unit disk. For the uncoupled case (with $K_{u}=K_{v}=0$ ), the surface diffusion coefficients and reaction kinetic parameters are chosen to ensure that there is a unique stable patternless solution for the reaction-diffusion system on the domain boundary. In addition, since typical surface diffusion coefficients are smaller than their bulk diffusion counterparts in applications, the condition $d_{u} \leq D_{u}$ and $d_{v} \leq D_{v}$ shall be imposed. Ultimately, the bifurcation analysis will illustrate how varying the ratio of bulk diffusivity and coupling coefficients can destabilize the system and lead to novel spatio-temporal dynamics. 


\subsection{Codimension-one bifurcation}

By numerically computing the roots of the eigenvalue relation (2.10), we can readily determine a linear stability diagram in the $D_{v}$ versus $K_{v}$ parameter space for the Schnakenberg and Brusselator boundary kinetics. For a circular harmonic mode with either $n=0,1,2$, the marginal stability curves in $D_{v}$ versus $K_{v}$ parameter space, for a fixed set of parameters, are shown in Fig. 1 and Fig. 2 for the Schnakenberg and Brusselator kinetics, respectively. In each phase diagram, the region of linear stability is located to the left of all the curves. Our computations show that the trivial mode $n=0$ loses stability through a Hopf bifurcation, while for the nontrivial modes $n=1,2$, stability is lost via a Turing bifurcation (zero-crossing eigenvalue).
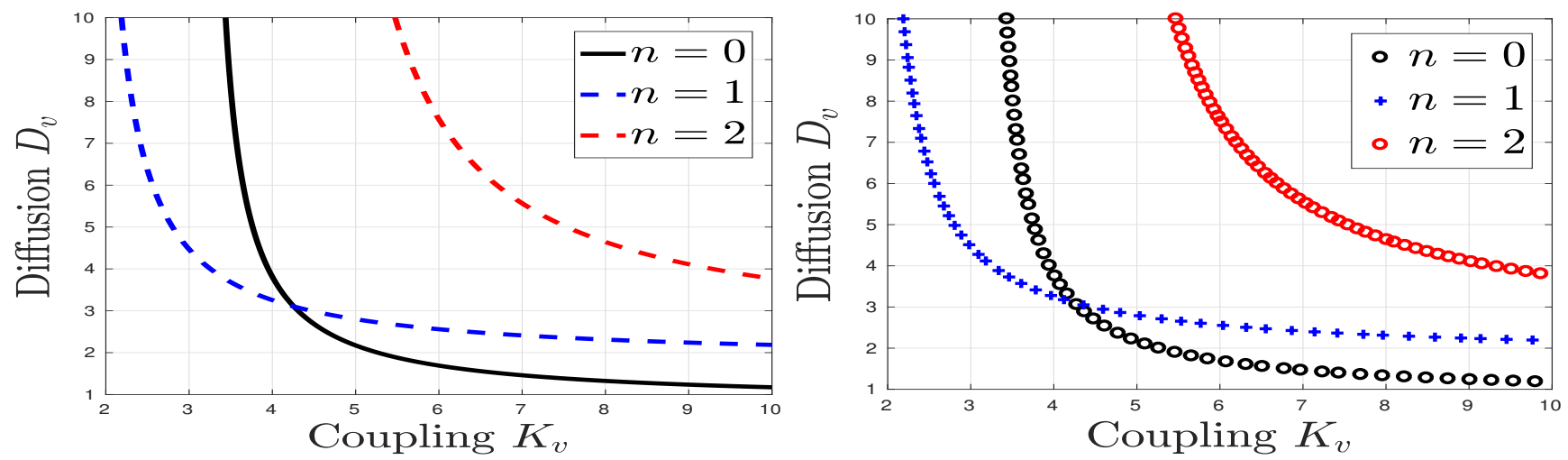

Figure 1: Linear stability diagram in the plane of parameters $\left(K_{v}, D_{v}\right)$ with Schnakenberg reaction kinetics. Other parameters are $R=1, D_{u}=1, \sigma_{u}=\sigma_{v}=0.01, K_{u}=0.1, d_{u}=d_{v}=0.1, a=0.1, b=0.9$. In the right panel, the symbol "o" indicates supercritical while "+" indicates subcritical.
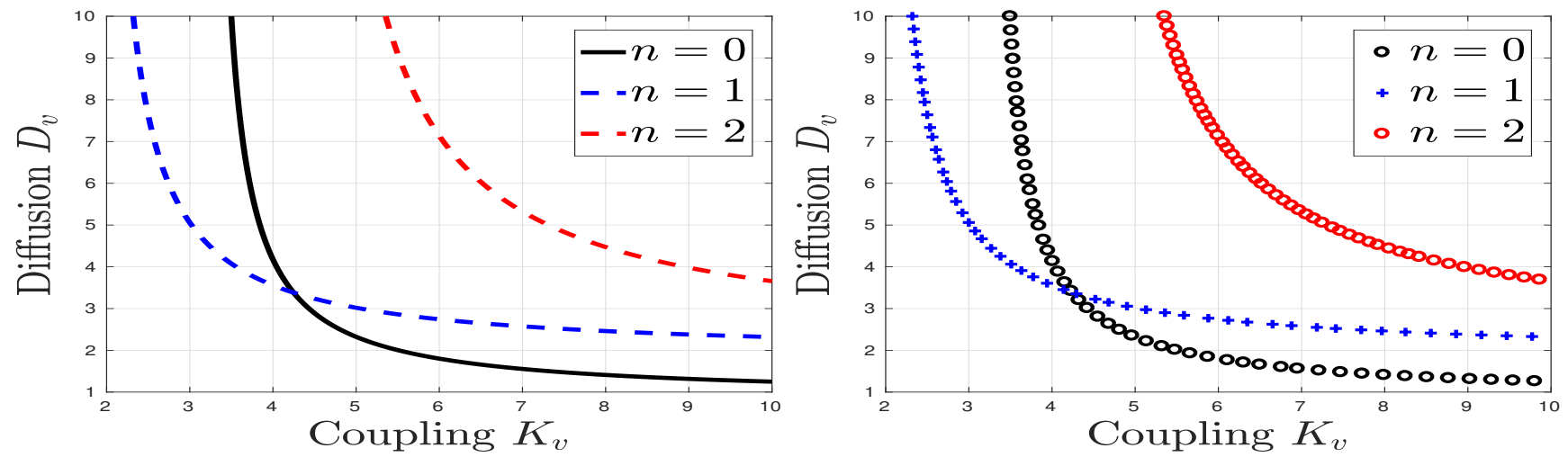

Figure 2: Stability diagram in the plane of parameters $\left(K_{v}, D_{v}\right)$ with Brusselator reaction kinetics. Other parameters are $R=1, D_{u}=1, \sigma_{u}=\sigma_{v}=0.01, K_{u}=0.1, d_{u}=d_{v}=0.5, a=3, b=7.5$. In the right panel, the symbol "o" indicates supercritical while " $"$ indicates subcritical.

Some qualitative trends are suggested from these linear stability phase diagrams. Firstly, when both bulk diffusion coefficients have the same order of magnitude, the Hopf bifurcation of the trivial mode is the dominant instability. It occurs when the coupling of the inhibitor is much larger than that of the activator, i.e. $K_{v} / K_{u} \approx 10^{2}$. Secondly, when this ratio decreases while the ratio $D_{v} / D_{u}$ increases, the primary instability switches to the Turing bifurcation of the first nontrivial mode $(n=1)$. This is reminiscent of the classical Turing paradigm for pattern formation, whereby the inhibitor is required to diffuse faster than the activator in order for a pattern to form.

The redrawn phase diagrams in the right panels of Fig. 1 and Fig. 2, indicating the local branching behavior of the bifurcation, was obtained after numerically evaluating the cubic term coefficients in the normal forms (2.80) and (2.81). For both reaction kinetics, supercritical Hopf and subcritical Turing bifurcations are predicted to occur as the stability curves associated with the modes $n=0,1$ are crossed. Although the transversal crossing of the rightmost curve associated with the mode $n=2$ is predicted to correspond to a supercritical Turing bifurcation, little attention is given to it in the subsequent discussion since it corresponds to a secondary instability.

We emphasize that our conclusions only hold for the current set of fixed parameters given in the captions of Fig. 1 and Fig. 2. In $\S 4.1 .2$ we show that a conclusion of super- or subcriticality can depend on the specific choices of reaction kinetic parameters and surface diffusion coefficients.

\subsubsection{Periodic solutions arising from Hopf bifurcations}

In this subsection the loss of stability through the supercritical Hopf bifurcation, as predicted by our weakly nonlinear analysis in Fig. 1 and Fig. 2, is investigated. Numerical continuation methods combined with PDE simulations are used to study the dynamics in the weakly nonlinear regime. As a result of azimuthal invariance, a simple 1-D finite difference 
method-of-lines approach is used to spatially discretize the coupled bulk-surface system, with the mean value theorem applied to derive an ODE for the bulk species at the origin that avoids the singularity inherent to the Laplacian in polar coordinates. Details of the discretization process are given in Appendix A.

We first study the loss of stability on the vertical line $K_{v}=5$ from Fig. 1 that intersects the $n=0$ Hopf curve at $D_{v} \approx 2.17$. Plots of global periodic solution branches using either $K_{v}$ or $D_{v}$ as the bifurcation parameter are displayed in Fig. 3. These numerical bifurcation diagrams confirm the prediction of the weakly nonlinear theory of a loss of stability through a supercritical Hopf bifurcation.
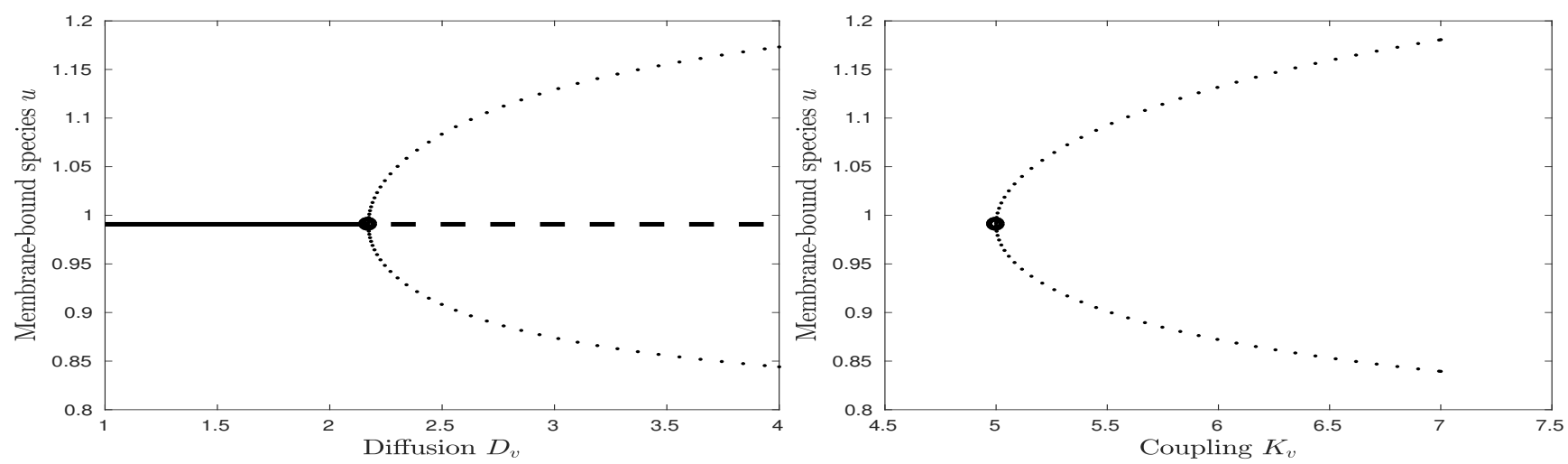

Figure 3: Global periodic solution branches computed with AUTO [6] past a supercritical Hopf bifurcation for the Schnakenberg kinetics. The continuation parameter in the left panel is $D_{v}$ with $K_{v}=5$, while in the right panel $K_{v}$ is used with $D_{v} \approx 2.17$. $N=200$ points discretize the radial direction.
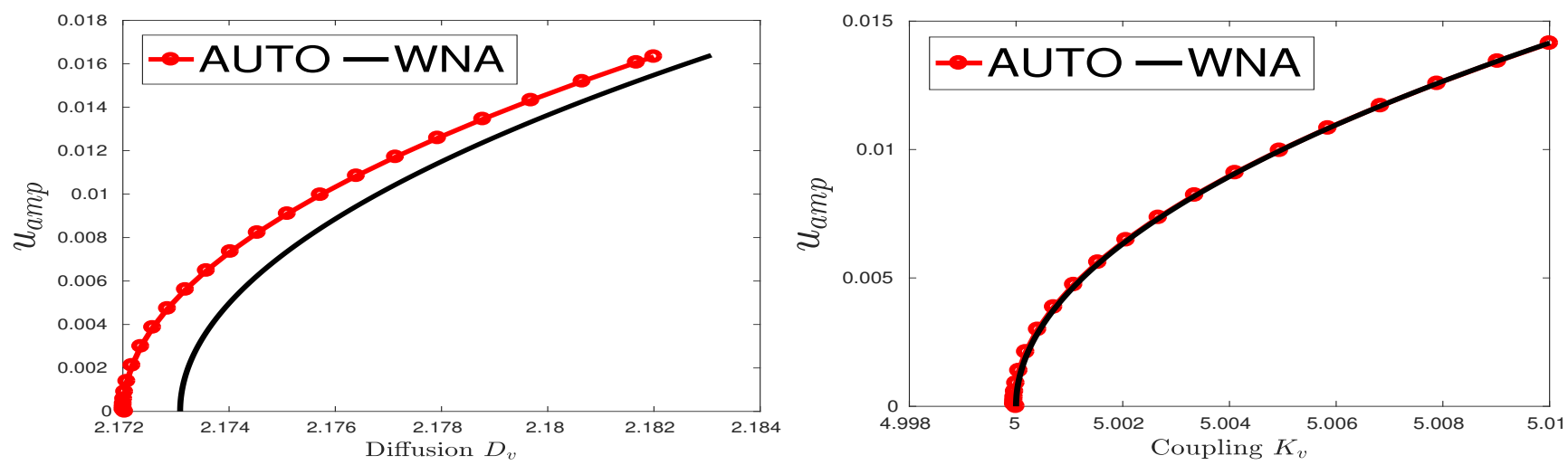

Figure 4: Amplitude of periodic solutions in the weakly nonlinear regime for the Schnakenberg reaction kinetics. The red curve is computed with AUTO using $N=200$ equidistant mesh points in the radial direction, while the black curve is obtained directly from the normal form (3.8) for $0 \leq \varepsilon \leq 0.1$.
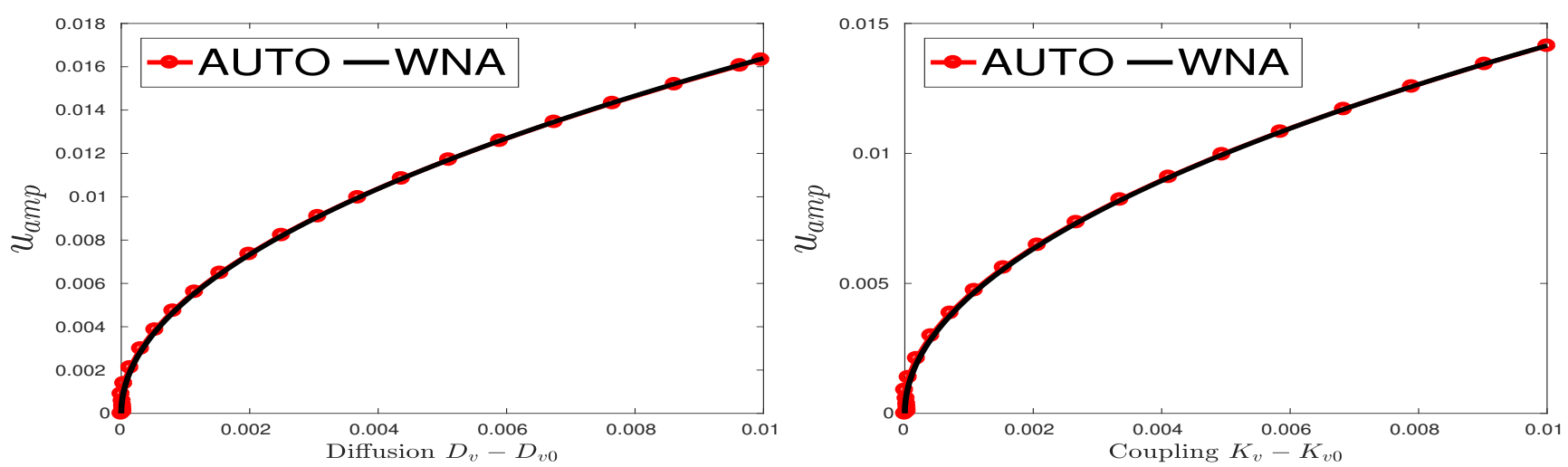

Figure 5: Translation to the origin of the branches in Fig. 4. The curvature of the bifurcating branches is correctly approximated by the weakly nonlinear theory.

Next, in the vicinity of the bifurcation point, (3.8) of Lemma 3.1 is used to predict the amplitude of the limit cycle. A graphical comparison between this predicted amplitude and corresponding numerical results computed with AUTO is shown in Fig. 4. In the left panel of Fig. 4 a slight shift in the bifurcation point caused by spatial discretization errors is observed. As the mesh is refined, the gap between the bifurcation points computed from the spatially discretized system and directly from the transcendental equation (2.10) by solving for pure imaginary roots is expected to shrink. This shift 
is not as apparent in the right panel of Fig. 4 since the bulk diffusivity $D_{v}$ was used to locate the Hopf bifurcation point. From Fig. 5, the two branches essentially coincide after translation to the origin.

As a further validation of the weakly nonlinear theory, we now compare the predicted period of oscillations near the Hopf point with corresponding numerical results extracted from PDE simulations. As shown in the right panel of Fig. 6 for a particular choice of detuning vector $\mu_{1}$ normal to the stability boundary (see the left panel of Fig. 6), the numerically computed period of oscillations is $T_{p} \approx 7.65$. This value agrees well with the result (3.9) from the weakly nonlinear theory.

Similar numerical experiments can be performed with the Brusselator reaction kinetics for the parameter set in the caption of Fig. 2. The results are qualitatively similar, with both the numerical results and the weakly nonlinear analysis predicting a loss of stability through a supercritical Hopf bifurcation. The periodic solution branches are displayed in Fig. 7 for the membrane-bound activator. Despite the slight shift in the bifurcation point, we observe a good agreement between the curvature of the branches computed with AUTO and from the weakly nonlinear theory (3.8). Moreover, at the bifurcation point, the magnitude of the eigenvalues is larger for the Brusselator than for the Schnakenberg model. This leads to a smaller oscillation period $T_{p} \approx 2.7719$ for the Brusselator. The corresponding full numerical simulation of the reduced PDE-ODE model is given in Fig. 8 for $\varepsilon=0.1$ and $\mu_{1}=(0,1)^{T}$.
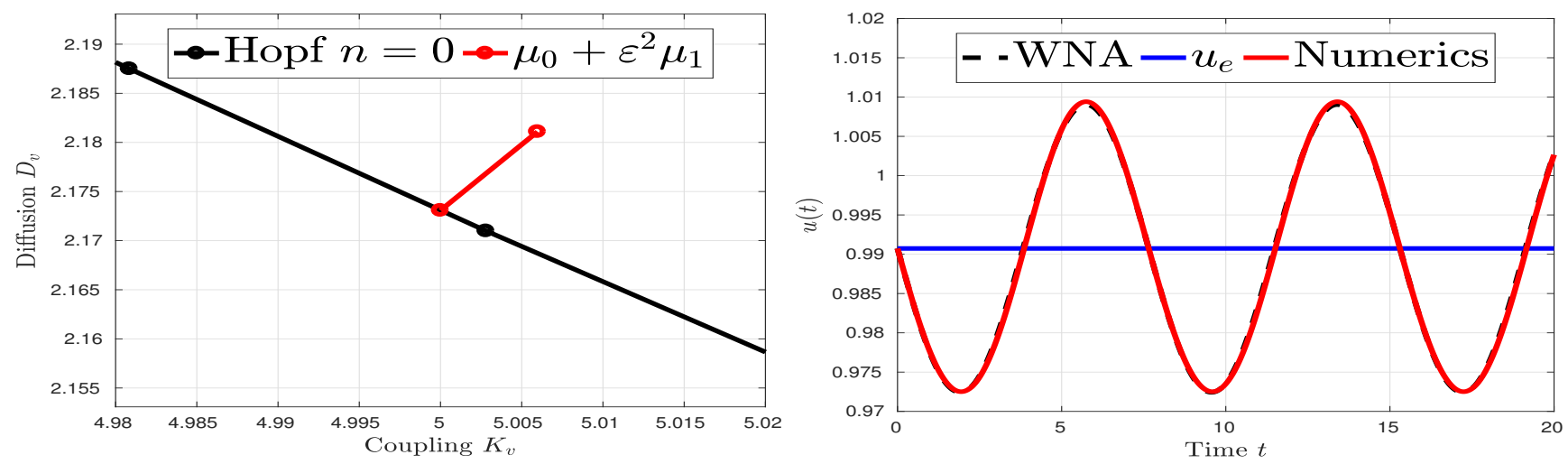

Figure 6: Full numerical simulation of the reduced PDE-ODE model with Schnakenberg kinetics. The right panel shows the membrane-bound activator $u(t)$ (red curve) which oscillates around the equilibrium solution (blue line). Notice here the good agreement with the solution in the weakly nonlinear regime (black dashed coinciding curve). Implicit-explicit time-stepping (SBDF2) [24] is employed from an initial condition given by (3.7) with $t=0, \theta_{0}(0)=\frac{\pi}{2}$ and $\varepsilon=0.1$. The bifurcation point and detuning vector respectively satisfy $\mu_{0}=(5,2.17)^{T}$ and $\mu_{1}=(0.6,0.8)^{T}$. The parameter values for the simulation are indicated in the left panel by a red dot.
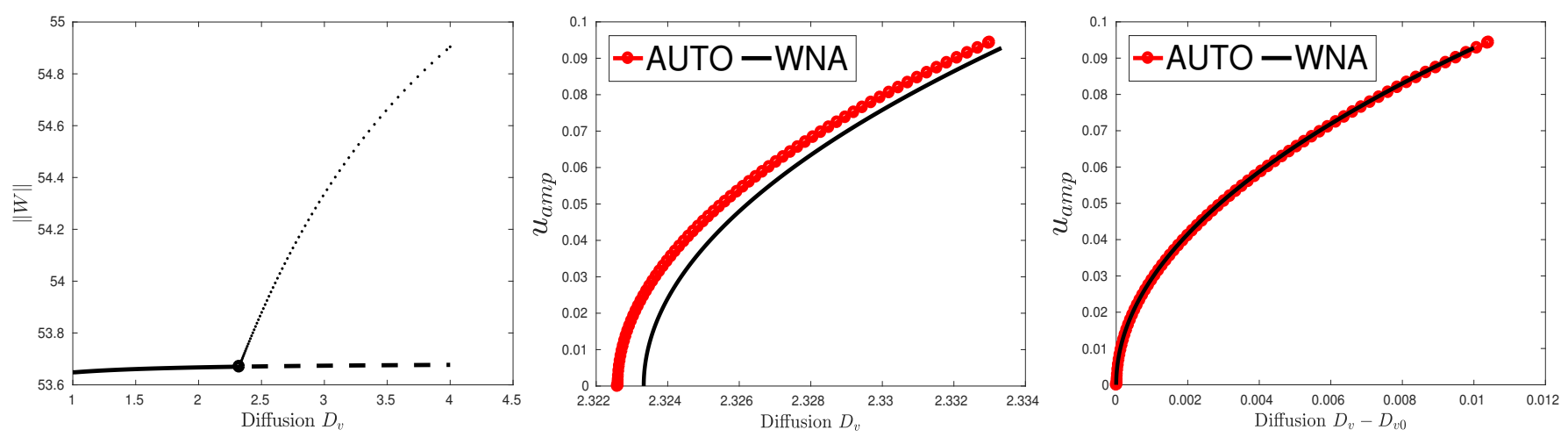

Figure 7: Periodic solution branches past a supercritical Hopf bifurcation with the Brusselator reaction kinetics. $N=200$ equidistant meshpoints discretize the radial direction for the radially symmetric reduced PDE-ODE model. Left panel: plot of the norm of the global solution branches computed with AUTO. Middle and right panels: local branching behavior predicted from the weakly nonlinear theory and from the bifurcation software AUTO are favorably compared.

As a result of the slight discrepancy between the bifurcation points computed in the spatially discretized system as compared to the weakly nonlinear theory, it is misleading to compare the result of a simulation with the analytical solution at a fixed parameter value. Therefore, a numerical convergence study as the mesh size $h=R /(N-1)$ decreases, with $N$ being the number of nodes used to discretize the radial direction (assuming azimuthal symmetry), is provided in Fig. 9. Since second-order centered differences are used, a quadratic rate of convergence is expected. Letting $\mu_{0}^{\text {num }}$ and $\mu_{0}^{w n a}$ denote, respectively, the bifurcation points in the spatially discretized versus continuous systems, we expect that

$$
\left\|\mu_{0}^{n u m}-\mu_{0}^{w n a}\right\|_{2} \leq \mathcal{O}\left(h^{2}\right)
$$

as $h$ tends to zero. This (roughly) quadratic convergence is confirmed in Fig. 9 where we computed the slope $\gamma$ of the two curves, characterizing the convergence rates, as

$$
\text { Schnakenberg (left): } \gamma \approx 2.078, \quad \text { Brusselator (right): } \gamma \approx 2.008 \text {. }
$$



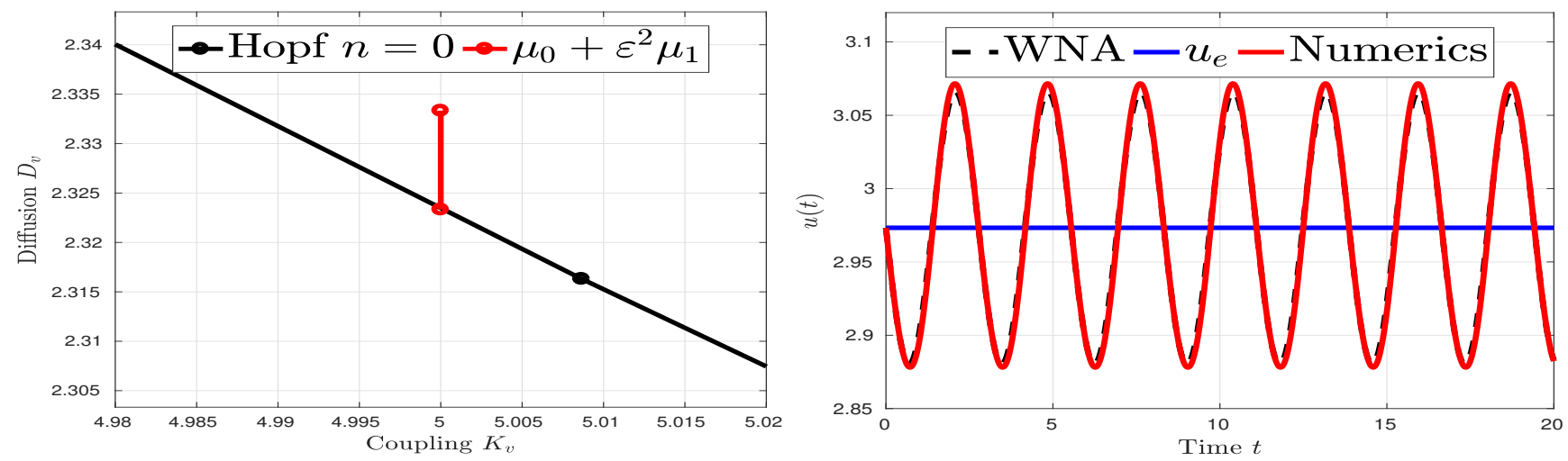

Figure 8: Full numerical simulation of the reduced PDE-ODE model with Brusselator kinetics. The same numerical method and initial condition used in Fig. 6 are employed here. The parameters are $\varepsilon=0.1, \mu_{0}=(5,2.32)^{T}$ and $\mu_{1}=(0,1)^{T}$. The red dot in the left panel indicates $\mu_{0}+\varepsilon^{2} \mu_{1}$, where the simulation is performed.
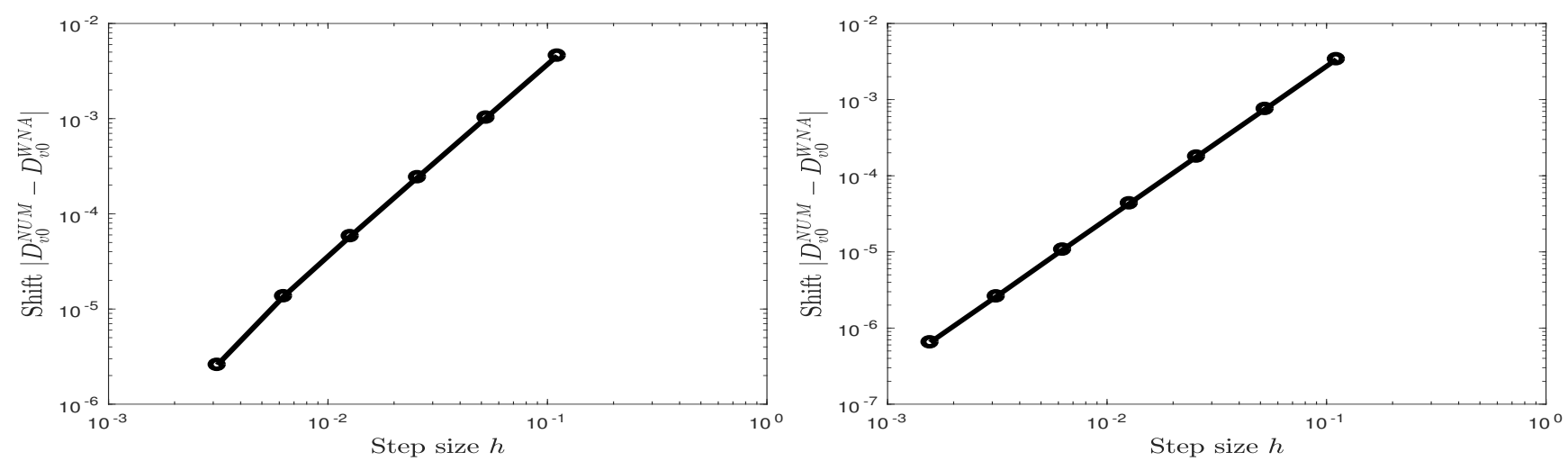

Figure 9: Convergence of numerically computed Hopf bifurcation points $D_{v 0}$ as the step size decreases for the Schnakenberg (left panel) and Brusselator (right panel) reaction kinetics with $K_{v}=5$ and all the other parameters being the same as in Fig. 1 and Fig. 2. Horizontal and vertical axis are displayed in a log scale. The computation of the bifurcation point in the spatially discretized system is performed with the software COCO [4]. The reference bifurcation point is obtained directly by solving the transcendental equation (2.10) for a pair of purely imaginary roots $\pm i \lambda_{I}$ and, therefore, is more accurate.

Next, we illustrate a delayed bifurcation behavior for the onset of oscillations. It is well-known that a slow sweep of a parameter through a Hopf bifurcation point can cause delayed transitions to oscillatory dynamics in systems of ODEs [1]. In order to observe such a delayed Hopf bifurcation effect in our PDE setting, the following numerical experiment was performed: We let $\kappa \ll 1$ be a second small parameter and $\mu(t) \in \mathbb{R}^{2}$ be a time-dependent vector of bifurcation parameters such that

$$
\mu(t)=\mu_{0}+(1-\kappa t) \varepsilon^{2} \mu_{1}
$$

Here, the parameter $\varepsilon$ does not need to be particularly small because the weakly nonlinear results from $\S 2$ and $\S 3$ do not apply to time-dependent bifurcation parameters. Full numerical results of the delayed bifurcation are shown in Fig. 10. The initial vector of bifurcation parameters is $\mu(0)=\mu_{0}-\varepsilon^{2} \mu_{1}$, for which the solution decays exponentially. The "static" bifurcation point is reached when $t=\kappa^{-1}$, after which the instability region is entered and sustained oscillations are expected. However, because of the slow parameter sweep, the transition to oscillations is delayed; an effect clearly observed in Fig. 10.

Next, we show that with Brusselator kinetics, there is a parameter regime where the Hopf bifurcation switches from supercritical to subcritical. A new linear stability phase diagram illustrating this transition is shown in Fig. 11. For this parameter set, the Hopf locus, provided by the trivial $n=0$ mode (black curve), is the primary instability. The same parameter values as given in the caption of Fig. 2 are used here, with the only difference being that the Brusselator kinetic parameter $b$ has been increased from $b=7.5$ to $b=8.7$. For this parameter set, we conclude that ratios of bulk diffusivity and coupling given by $D_{v} / D_{u} \gg 1$ and $K_{v} / K_{u} \approx 1$ are sufficient for the Hopf bifurcation to be subcritical. Alternatively, when $D_{v} / D_{u} \approx 1$ and $K_{v} / K_{u} \gg 1$, the Hopf bifurcation is supercritical. This criticality change is also shown in Fig. 12, where we clearly observe that the real part of the cubic term coefficient in (2.80) changes sign at some point along the Hopf locus.

To confirm this transition to a subcritical Hopf bifurcation as predicted from our weakly nonlinear theory, we perform numerical simulations near the intersection point of the Hopf locus in Fig. 11 and the horizontal line $D_{v}=9$. The global bifurcating branch computed with AUTO is shown in the left panel of Fig. 13. In the right panel of Fig. 13 we plot the corresponding numerically computed period of oscillations. These numerical results confirm the predicted loss of stability through a subcritical Hopf bifurcation. The results also suggest bistability between a large amplitude limit cycle and the steady state solution in a small parameter window prior to the bifurcation point. From the left panel of Fig. 13, the 

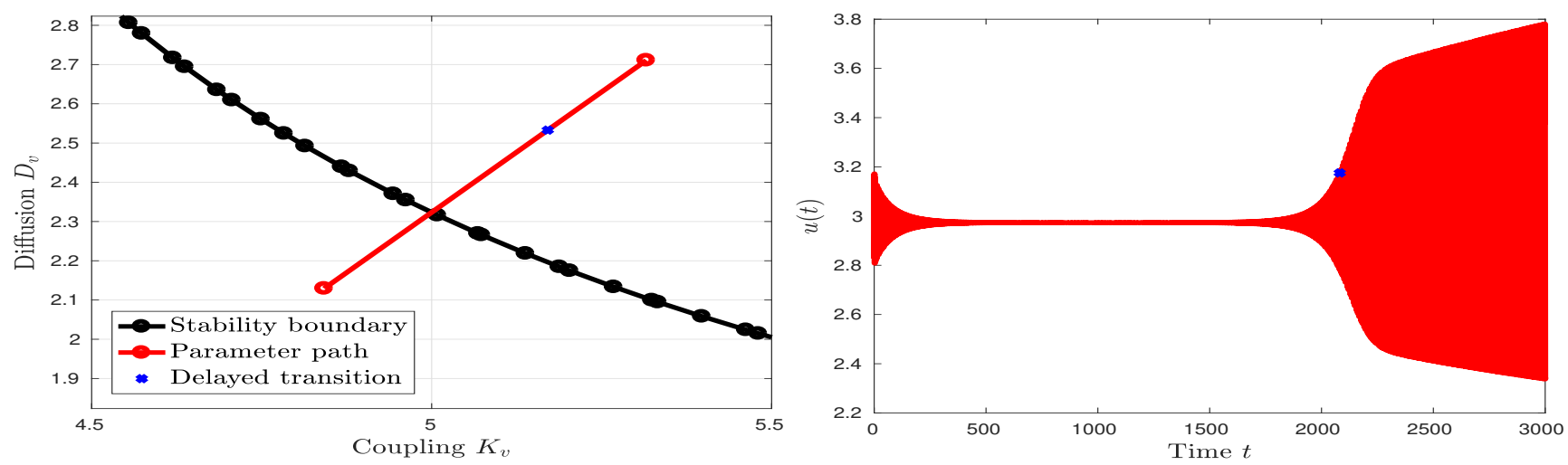

Figure 10: Delayed Hopf bifurcation for the Brusselator kinetics with $\kappa=10^{-3}, \varepsilon=0.5, \mu_{0}=(5,2.32)^{T}$ and $\mu_{1}=$ $(0.63,0.78)^{T}$ (see $\left.(4.3)\right)$. The left panel shows the path of the time-dependent parameter sweep, with the initial and final points being in the stability (bottom left) and instability (up right) regions, respectively. Numerical results for the membrane-bound activator $u(t)$ are shown in the right panel. The "static" bifurcation point is reached when $t=1000$, but the transition to a periodic solution is delayed. The reduced PDE-ODE model (because of azimuthal invariance) is discretized using the method of lines. The stiff ODE MATLAB solver ode23s is used for the numerical time integration.


Figure 11: Stability curves associated with the modes $n=0,1,2$ in the plane of parameters $\left(K_{v}, D_{v}\right)$ for the Brusselator kinetics. Other parameter values are $R=1, D_{u}=1, \sigma_{u}=\sigma_{v}=0.01, K_{u}=0.1, d_{u}=d_{v}=0.5, a=3$ and $b=8.7$. The region of linear stability is located to the left of the $n=0$ stability boundary, which corresponds to a locus of Hopf bifurcations. In the right panel, the symbol 'o' indicates a supercritical bifurcation while '+' indicates a subcritical bifurcation. In the right panel, notice the transition from sub to super-criticality along the $n=0$ Hopf locus (black curve).

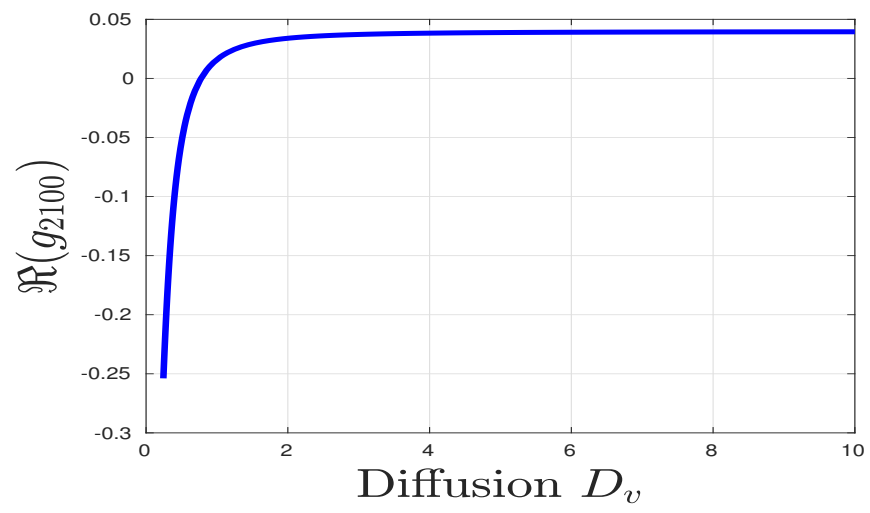

Figure 12: Transition from a supercritical to a subcritical Hopf bifurcation for the Brusselator kinetics. The plot shows that the real part of the coefficient $g_{2100}$ in (2.80), when numerically evaluated along the Hopf stability curve in Fig. 11, exhibits a sign-change. 
stable and unstable branches of periodic solution merge at a fold point around $K_{v} \approx 0.8$.
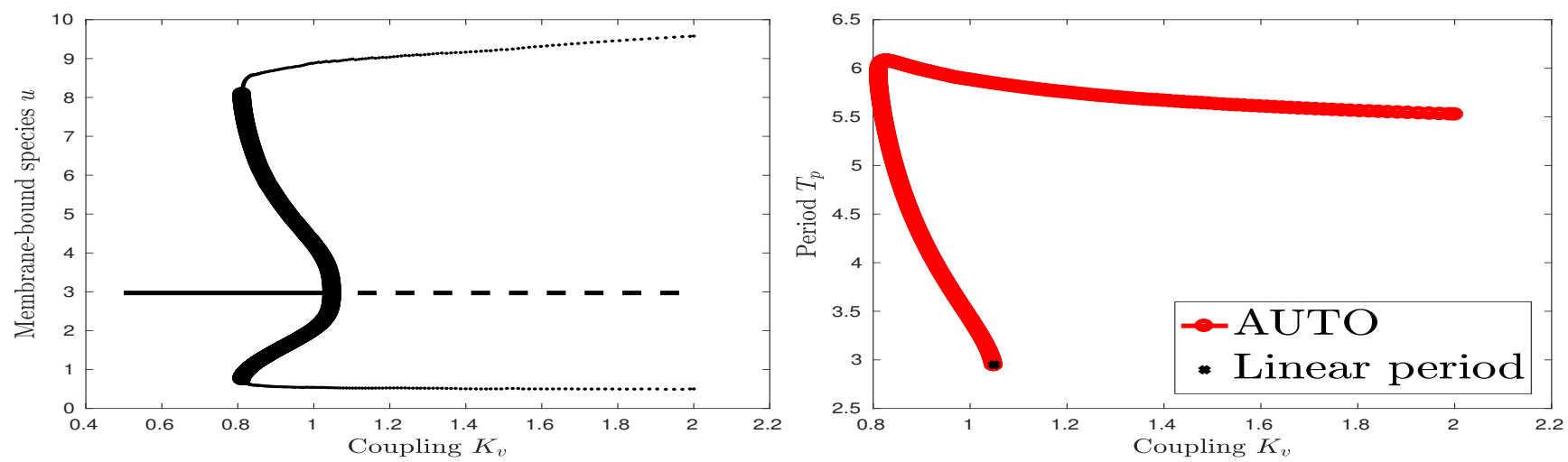

Figure 13: The left panel shows the global bifurcating branch for the membrane-bound activator species. The right panel shows the numerically-computed period as a function of the coupling $K_{v}$ when $D_{v}=9$ (other parameters as in the caption of Fig. 11). Notice that the initial point matches the linear period predicted by the asymptotic theory, indicated by a black "x". The computation is performed with AUTO using $N=200$ radial grid points in the bulk.

In Fig. 14, we compare the numerically computed amplitude of oscillations against results from our weakly nonlinear theory for $\varepsilon=0.025$. Despite the slight shift between the bifurcation points, good agreement is once again obtained. However, we notice that the range over which the two branches coincide is much more narrow than for their supercritical counterparts. This is likely due to the real part of the cubic term coefficient in (2.80) having a rather small magnitude when $D_{v}=9$ (see Fig. 12), which suggests that the unresolved quintic term in the normal form may be quantitatively significant.

Finally, in Fig. 15 we show numerical PDE results of large amplitude relaxation oscillations that can occur on the horizontal line $D_{v}=9$ in Fig. 11. These oscillations, characterized by sharp variations followed by a rest period, are often observed in simpler ODE models having a subcritical Hopf bifurcation. They are qualitatively distinct from the harmonic-type oscillations in Fig. 6 and Fig. 8.

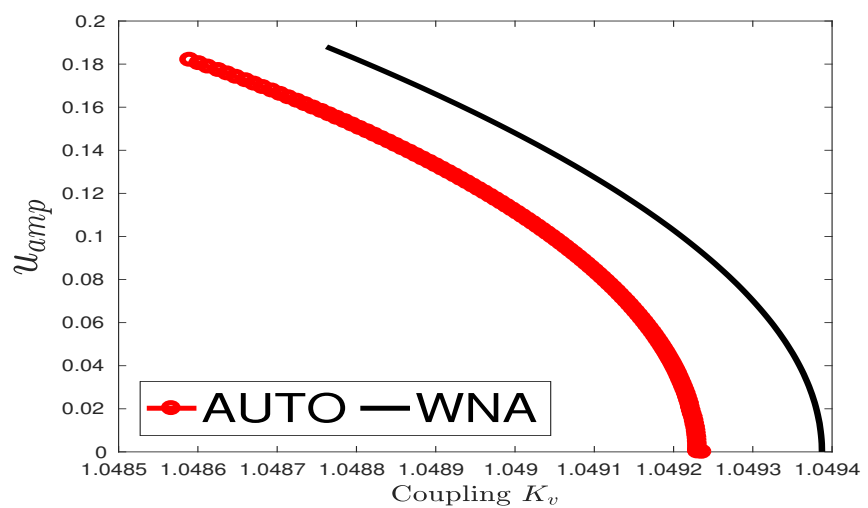

Figure 14: Local unstable periodic solution branch past a subcritical Hopf bifurcation for the Brusselator kinetics with $D_{v}=9$ (other parameters as in the caption of Fig. 11). The red curve is obtained through numerical continuation using AUTO, while the black curve is the amplitude $u_{a m p}$ as predicted by the weakly nonlinear theory in (3.8) with $\varepsilon=2.5 \times 10^{-2}$. 

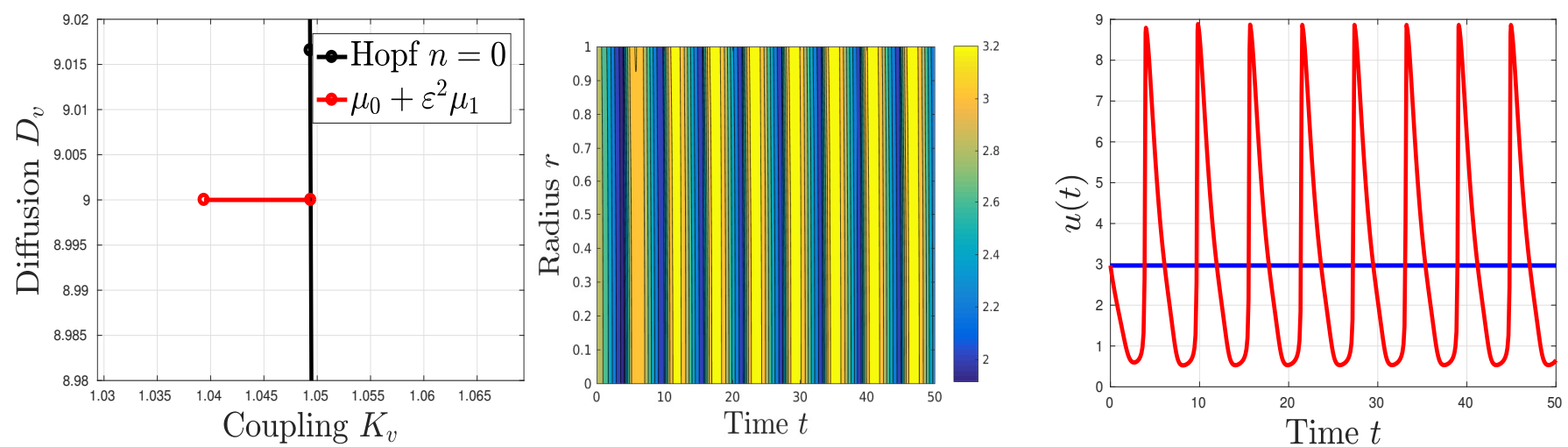

Figure 15: Highly nonlinear relaxation-type oscillations near a subcritical Hopf bifurcation for the Brusselator kinetics. As shown in the left panel, the simulation is performed on the horizontal line $D_{v}=9$ with $\varepsilon=0.1$. In the middle panel, the numerically computed spatio-temporal bulk oscillations are plotted for the activator species $U(r, t)$, with the radius $r$ on the vertical axis and time $t$ on the horizontal axis. The right panel shows the corresponding relaxation oscillations for the membrane-bound activator species. The initial condition corresponds to the unstable periodic solution in the weakly nonlinear regime as given by (3.7) with $\theta_{0}(0)=\frac{\pi}{2}, t=0, \varepsilon=0.1$.

\subsubsection{Turing patterns arising from pitchfork bifurcations}

In this subsection the formation of spatially inhomogeneous steady states close to a pitchfork (or Turing) bifurcation is investigated numerically. Since there is no azimuthal invariance near such bifurcations, one cannot use 1-D finite differences to spatially discretize the system as in $\$ 4.1 .1$. Hence, the full spatial structure of the model must be considered, and its discretization is done with the finite element method as implemented by the PDE Toolbox of MATLAB [21]. To be more precise, linear triangular elements are used to discretize the bulk domain, while the 1-D Laplace-Beltrami boundary diffusion operators is handled with second-order centered differences using the nodes attached to the boundary.

As mentioned in [2], the computation of general equilibria to system of elliptic PDEs posed on arbitrary 2-D or 3-D domains is a challenging task. For these domains, the spatial discretization yields large and sparse systems of nonlinear equations for which traditional software like AUTO [6] and MATCONT [5] are of limited use. It is to address these issues that a number of new MATLAB packages such as pde2path [25] and Computational Continuation Core (COCO) [4] have emerged in the research community. While pde2path has been specifically designed for systems of elliptic PDEs, it cannot handle nonstandard boundary conditions like those encountered in bulk-surface coupled models. The base state can be computed using the Equilibrium Point toolbox from COCO, but our attempt to compute the global bifurcating branch at a pitchfork bifurcation point has been unsuccessful. From a numerical bifurcation analysis perspective, the situation is rather degenerate since rotational symmetry results in two critical eigenfunctions at the pitchfork bifurcation point. Consequently, the results exhibited in this section mostly rely on full numerical time-dependent simulations using Implicit-Explicit time-stepping; an approach that unfortunately only reveals stable steady states (either patterned or patternless). In practice, the simulation is stopped when the relative distance between the current and the previous time step becomes smaller than some given tolerance. More details regarding the spatial discretization and the numerical methods are given in Appendix A.

First, the loss of stability of the modes $n=1$ through subcritical pitchfork bifurcations as the coupling rate $K_{v}$ increases is investigated numerically. The reader is referred to Fig. 1 and Fig. 2, where we consider the horizontal line $D_{v}=5$ and its intersection with the $n=1$ pitchfork curve. In the right panel of Fig. 16 the amplitude of the membrane-bound activator species for the Schnakenberg kinetics is shown. The black curve is the unstable bifurcating branch and is only valid locally. In theory, the unstable (black) and the stable (red) branches should merge at a turning (or fold) point near $K_{v} \approx 2.7976$. Such a feature cannot be detected with the weakly nonlinear analysis from $\S 2$ or with direct time-stepping numerical simulations. Instead, numerical continuation methods must be employed. Having discussed the challenges associated with such a task earlier in this subsection, the computation of the full branch is an open problem. Nevertheless, the solution in the weakly nonlinear regime can be used as an initial condition for a direct numerical simulation, with the anticipation that it evolves to the stable branch. The result of such an experiment is shown in Fig. 17 for $\varepsilon=0.01$.

The results of similar experiments using the Brusselator kinetics with $D_{v}=5$ (see the phase diagram in Fig. 2) are shown in Fig. 18 and Fig. 19. Notice here that the unstable branch goes farther backward than in the Schnakenberg case before reaching a "turning point" at around $K_{v} \approx 2.66$. The pitchfork bifurcation point is at $K_{v 0} \approx 3.02$. Again, this is an example of a hard loss of stability of the base state.

Despite being unable to compute bifurcating branches using numerical continuation methods, the package coco (cf. [4]) can be used to estimate the shift in bifurcation points between the full model and its finite element discretization. In Fig. 20 we show the results of such a convergence study, where $h_{\max }$ is the maximal distance between two nodes on the mesh. Letting $N$ be the number of equidistant nodes on the circular boundary, then $h_{\max }$ is $\operatorname{chosen}$ as $h_{\max }=2 \pi R / N$. As $h_{\max }$ tends to zero, the discrepancy between the bifurcation points is expected to converge like

$$
\left\|\mu_{0}^{n u m}-\mu_{0}^{w n a}\right\|_{2} \leq \mathcal{O}\left(h_{\max }^{\gamma}\right),
$$



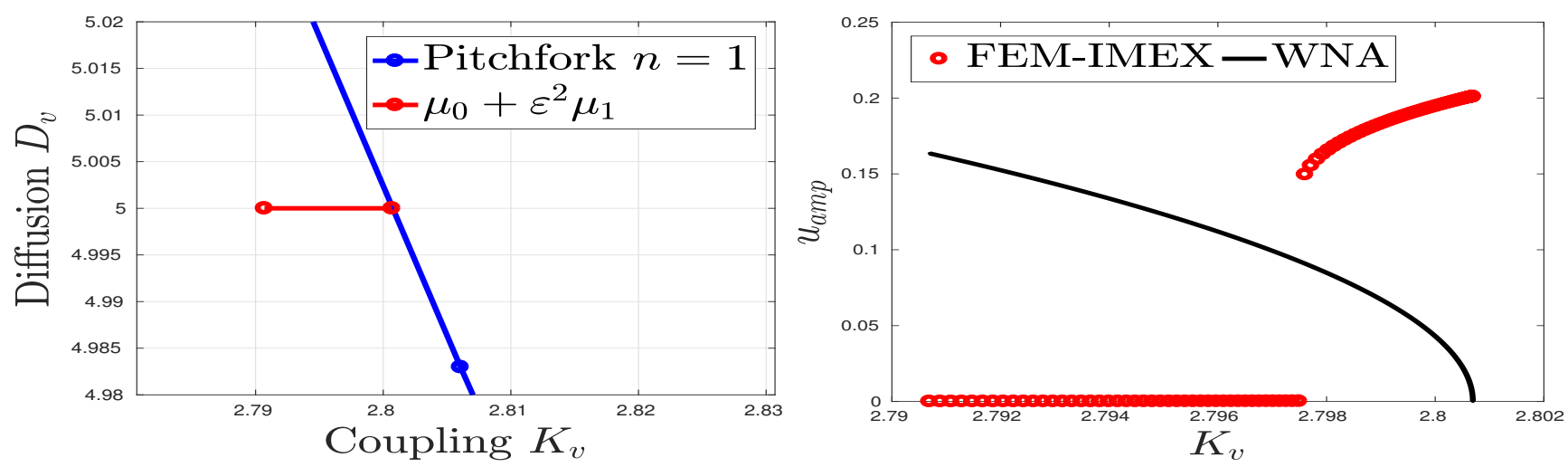

Figure 16: Subcritical pitchfork bifurcation with the Schnakenberg kinetics and $D_{v}=5$ (the other parameters are given in the caption of Fig. 1). The left panel is a magnified version of the stability diagram near the bifurcation point for $\varepsilon=0.1$. The stability region is located to the right of the blue curve, while the red curve indicates the parameter path. The right panel displays the maximal amplitude of the membrane-bound activator, with the black curve obtained from the weakly nonlinear theory (2.81) while the red curve is computed through successive numerical simulations. The boundary of the circular bulk domain is discretized with $N=200$ nodes.
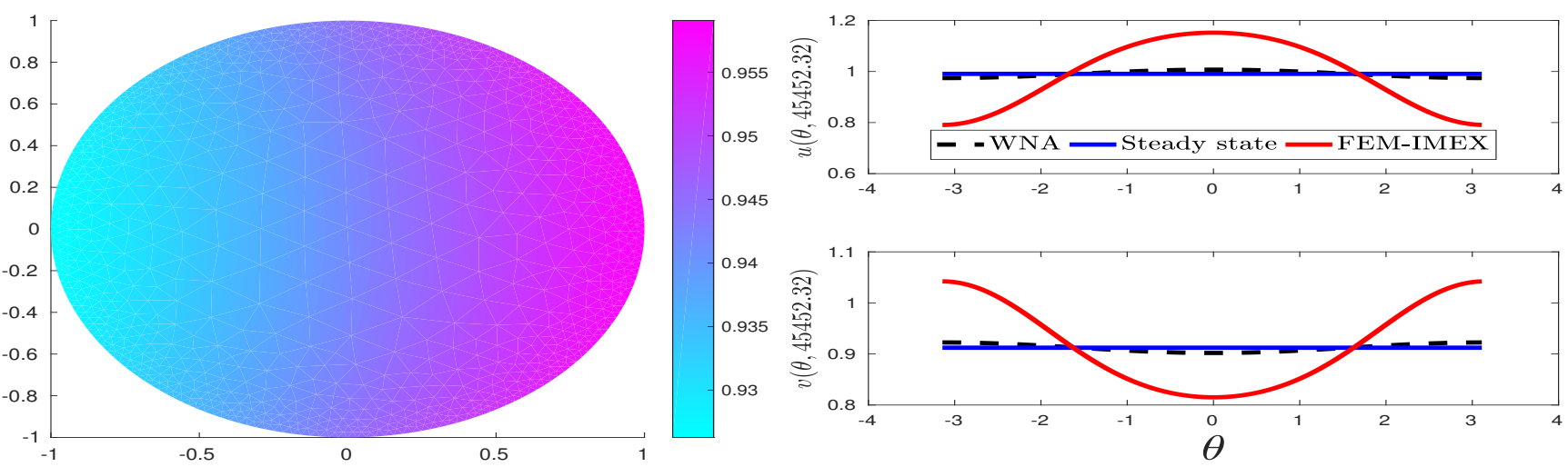

Figure 17: A numerically computed stable pattern for $t \approx 45452$ in the vicinity of a subcritical pitchfork bifurcation under Schnakenberg kinetics. The black dashed curves in the right panel correspond to the unstable membrane-bound patterns, while the red curves correspond to the stable patterns. The solution in the weakly nonlinear regime, as given by (3.15) with $\varepsilon=0.01, n=1$ and $\theta_{n}=0$, is used as an initial condition. Because the critical eigenvalues are very small near the bifurcation point, the numerical solution only very slowly reaches the stable patterned state.
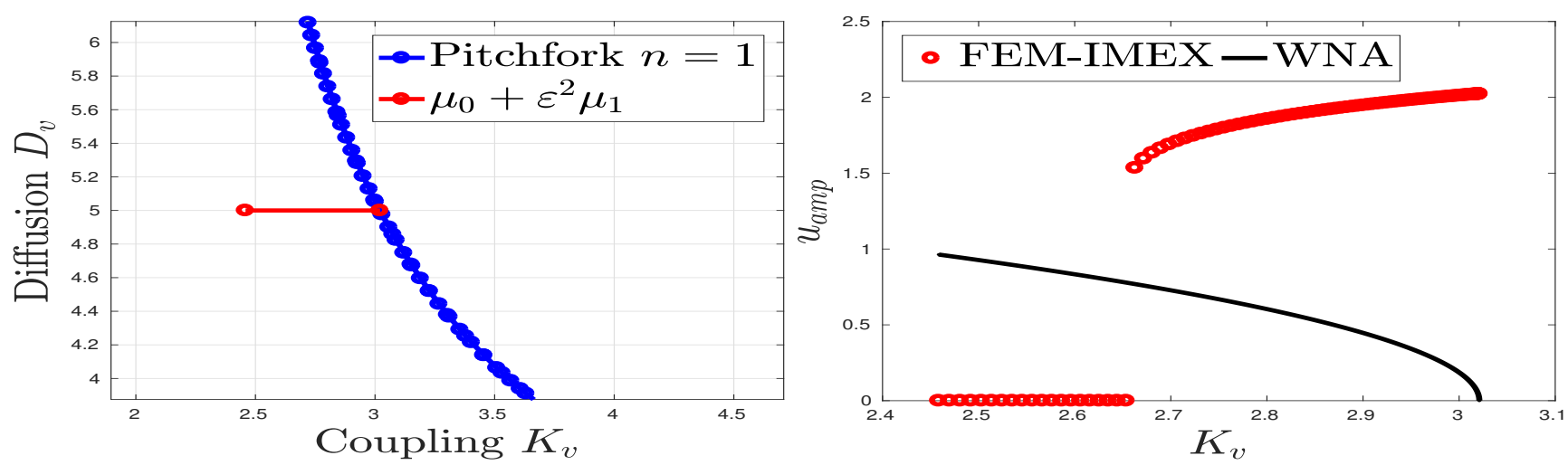

Figure 18: Subcritical pitchfork bifurcation with the Brusselator kinetics, $D_{v}=5$, and $\varepsilon=0.75$ (the other parameters are given in the caption of Fig. 2). Again, the unstable branch goes backward, under which the base state solution is linearly stable. The boundary of the circular bulk domain is discretized with $N=200$ nodes. 
for some positive power $\gamma$. Estimating the slope of the curve in the right panel of Fig. 20 yields $\gamma \approx 1.97$. Similar quadratic convergence rate was obtained for bifurcation points of systems with radial symmetry discretized with simple finite differences (see $\S 4.1 .1)$.
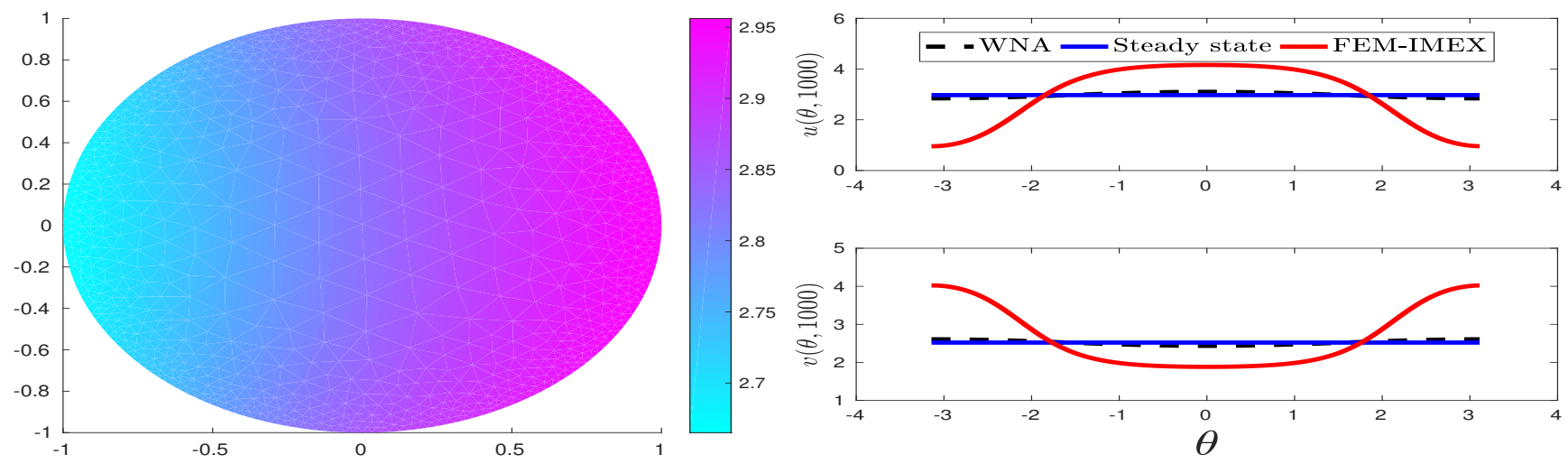

Figure 19: A numerically computed stable pattern at $t=1000$ (red curve), near the steady state, as evolved from the unstable branch near a subcritical pitchfork bifurcation with the Brusselator kinetics and $\varepsilon=0.1$. The left panel shows the corresponding solution in the bulk.
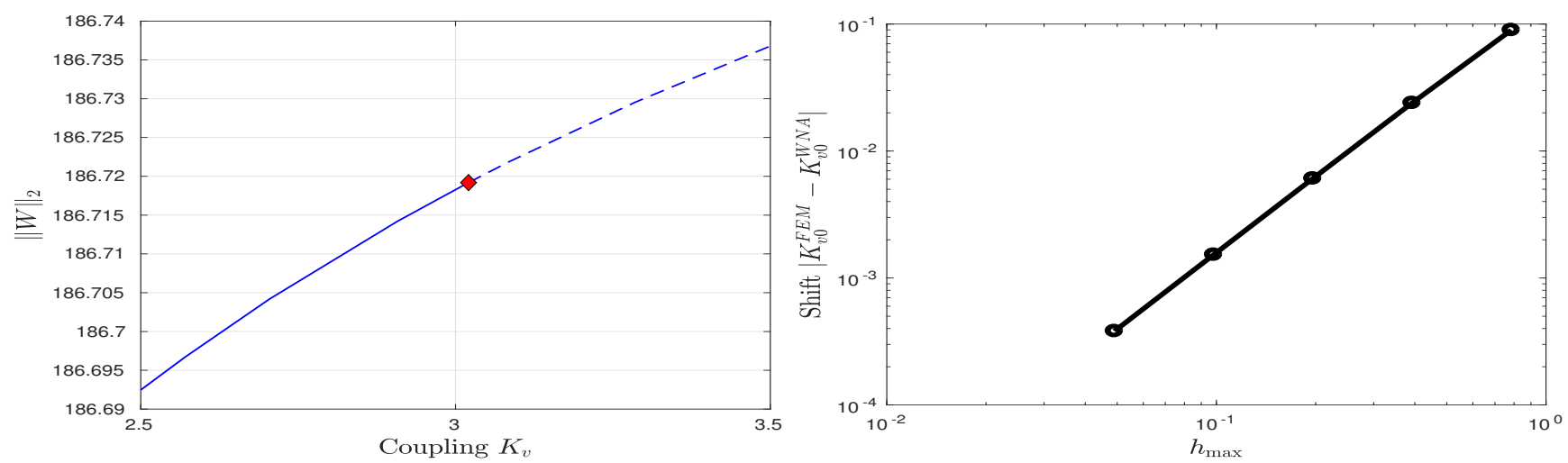

Figure 20: Convergence of pitchfork bifurcation points between the continuous versus the spatially discretized system on the vertical line $D_{v}=5$ for the Brusselator kinetics (see the plots in Fig. 2). The right panel plots on a logarithmic scale the distance between the bifurcation points in the discrete versus continuous systems as $h_{\max }$ tends to zero. The left panel shows the standard Euclidean norm of the base state solution as the parameter $K_{v}$ increases past the pitchfork bifurcation point when $N=128$ nodes are used on the boundary, yielding a maximal distance of $h_{\max } \approx 4.9 \times 10^{-2}$.

In a different parameter regime, we now show that under Brusselator kinetics the branching behavior at the pitchfork point can be supercritical instead of subcritical. For the parameter set in Fig. 21 a new pitchfork bifurcation locus is plotted in the $D_{v}$ versus $K_{v}$ parameter plane. By numerically evaluating the cubic coefficient of the normal form (2.81), the weakly nonlinear theory from $\S 2$ now predicts a supercritical pitchfork bifurcation (see the right panel of Fig. 21). In comparison with the parameter set used for the subcritical case in Fig. 2, we took a slightly different value for $b$ in the Brusselator kinetics, while the surface diffusion coefficients were increased to $d_{u}=d_{v}=1$.

To validate the prediction of supercriticality, numerical bifurcation results and full PDE simulations are undertaken near a bifurcation point on the stability boundary. In the right panel of Fig. 22 the amplitude of the patterned state for the membrane-bound activator when $K_{v}$ increases on the horizontal line $D_{v}=15$ is shown. Here, a rather close agreement between (2.81) from the weakly nonlinear theory and the numerical bifurcation results is obtained because the bifurcating branch is stable. Moreover, as predicted by the theory, the amplitude of the patterned state scales as the square root of the distance from the bifurcation point. The corresponding stable pattern computed from full PDE simulations with $\varepsilon=0.1$ is shown in Fig. 23 and favorably compared with results from the weakly nonlinear theory.

Next, we give a convergence study comparing $W_{F E M}$, the numerical solution to the spatially discretized system, with the leading-order asymptotic solution (3.15) in the weakly nonlinear regime, and denoted by $W_{W N A}$. Such an experiment is only valid when testing a stable patterned solution arising from a supercritical pitchfork bifurcation. Referring to Fig. 23, the stable pattern for $\varepsilon=0.1$ is repeatedly computed while decreasing $h_{\max }$ on a uniform grid. For each $h_{\max }$ value, the error is estimated using the weakly nonlinear asymptotic solution as a substitute for the unknown exact solution.

A plot of the error as a function of $h_{\max }$ is shown in Fig. 24, where two different solution measures are employed. Assuming sufficient regularity of the exact solution, and given that the mesh consists of linear triangular elements, the error is expected to behave like

$$
\left\|W-W_{F E M}\right\|_{L^{\infty}} \leq \mathcal{O}\left(h_{\max }^{2}\left|\log h_{\max }\right|\right), \quad \text { as } \quad h_{\max } \rightarrow 0
$$



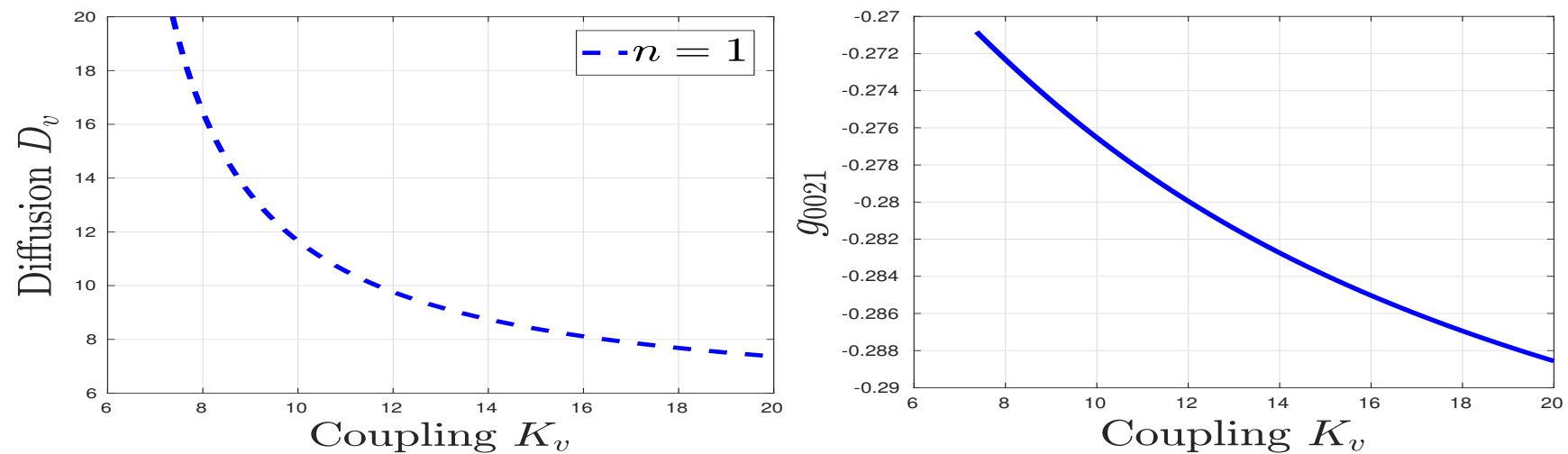

Figure 21: Left panel: the pitchfork bifurcation $\left(n=1\right.$ mode) curve in the $D_{v}$ versus $K_{v}$ parameter plane for Brusselator kinetics with $a=3$ and $b=5$. Other parameter values are $R=1, D_{u}=1, \sigma_{u}=\sigma_{v}=0.01, K_{u}=0.1, d_{u}=d_{v}=1$. The region of linear stability is to the left of the curve. Right panel: the corresponding coefficient $g_{0021}$ of the cubic term in the normal form (2.81), along the pitchfork bifurcation locus. This coefficient is negative, indicating a supercritical pitchfork bifurcation.
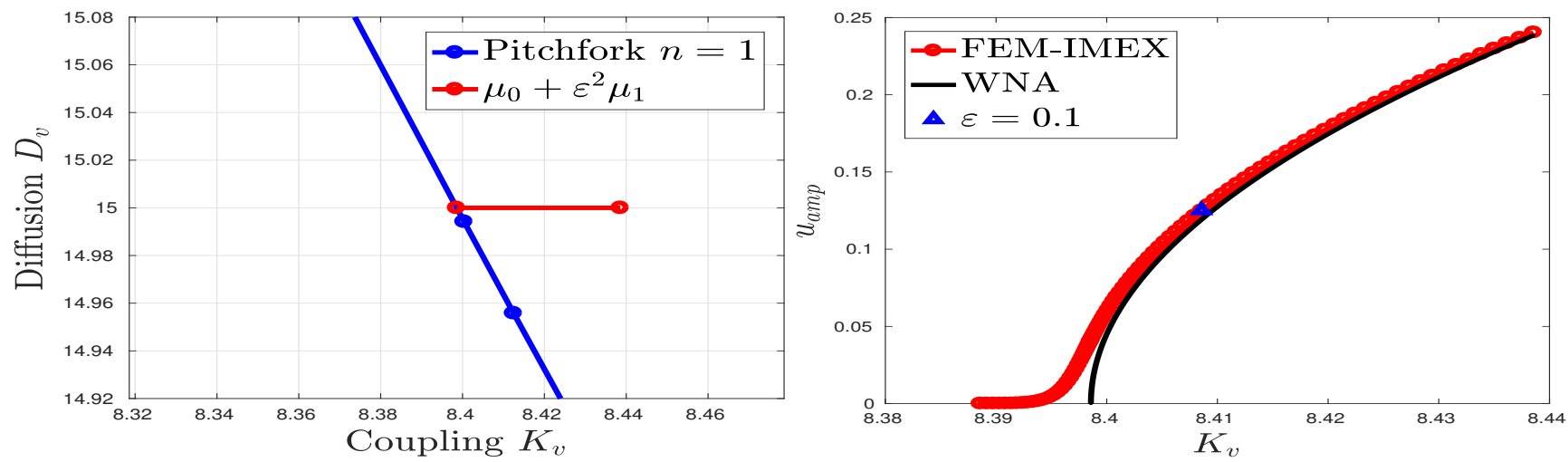

Figure 22: Stable bifurcating branch past a supercritical pitchfork bifurcation for the Brusselator kinetics, with the rightmost point corresponding to $\varepsilon=0.2$. The parameters are as given in Fig. 21. The left panel shows the parameter path past the bifurcation point. The right panel compares $u_{a m p}$, as obtained from the weakly nonlinear theory (2.81), with numerically computed results from PDE simulations.
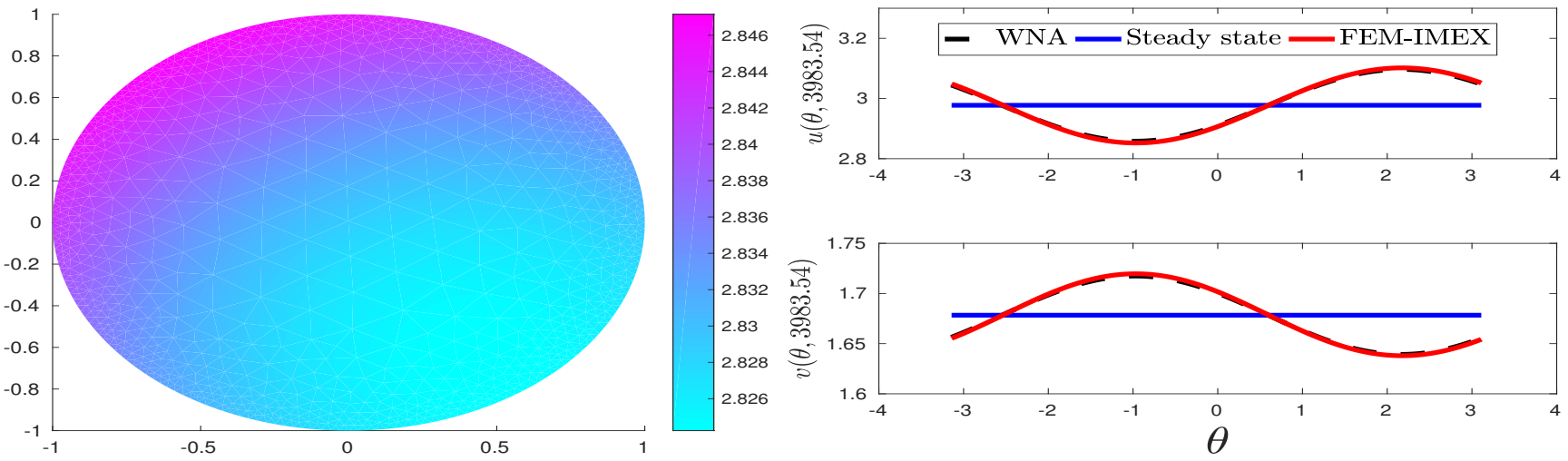

Figure 23: Stable pattern near a supercritical pitchfork bifurcation of the $n=1$ mode with $\varepsilon=0.1$, corresponding to the point indicated by a blue triangle on the bifurcation branch plotted in the right panel of Fig. 22. Left panel: contour plot of the activator concentration in the bulk $(U)$. Right panel: membrane-bound patterns. A close agreement between the weakly nonlinear theory predicted by (3.15) (black dashed curve) and the (nearly coinciding) numerical PDE results (red curve) is obtained. Here the nonzero phase $\theta_{n}$ in (3.15) was calculated from the numerical solution, whose initial condition is a linear combination of the base state solution with the critical eigenvectors of the Jacobian of the spatially discretized system. 
using the $L^{\infty}$ norm (see remark 4.41 in [12]). For the $L^{2}$ norm, quadratic convergence rate is expected (see Theorem 4.34 in $[12])$

$$
\left\|W-W_{F E M}\right\|_{L^{2}} \leq \mathcal{O}\left(h_{\max }^{2}\right), \quad \text { as } \quad h_{\max } \rightarrow 0 .
$$

The plots in Fig. 24 confirm the bounds given in (4.5) and (4.6), with the error as measured with the $L^{\infty}$ norm (right panel) converging slightly faster than expected. The choice of a temporal discretization, along with an associated stopping criteria for the solver, may influence the convergence of the numerical solution. Although it should not affect the speed of convergence, the error estimates are also biased since we are using the leading-order asymptotic solution (3.15) as a proxy for the exact solution.
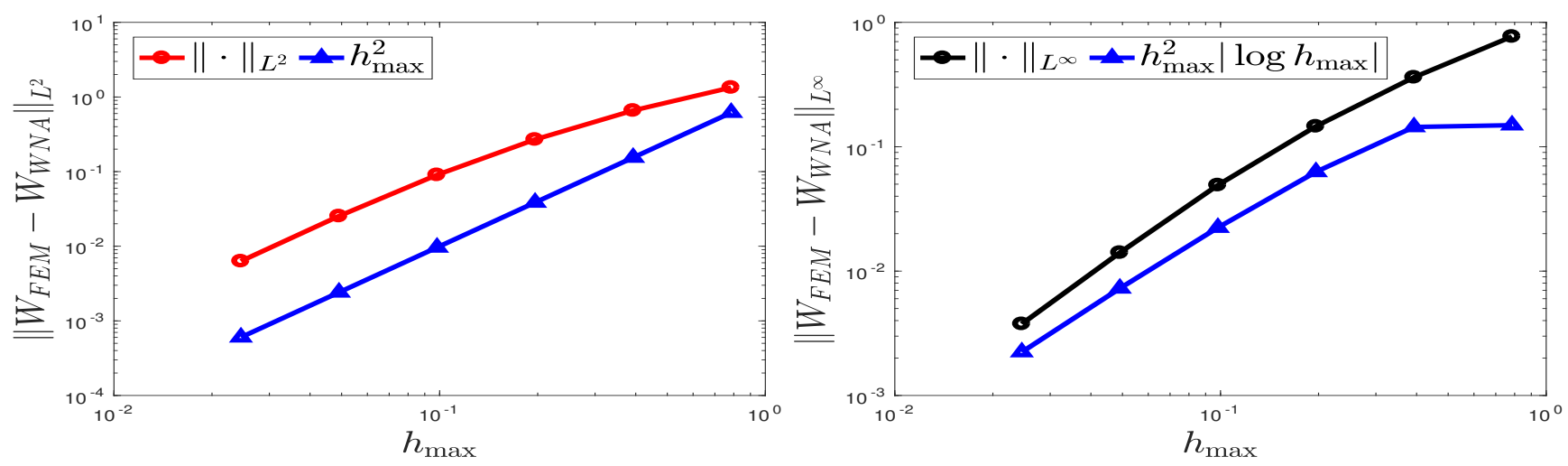

Figure 24: Convergence of the numerical solution as $h_{\max }$ approaches zero. Left panel: error measured with the $L^{2}$ norm (red curve) is compared with $h_{\max }^{2}$. Right panel: the error in the $L^{\infty}$ norm (black curve) is compared with $h_{\max }^{2}\left|\log h_{\max }\right|$. The approximate weakly nonlinear solution is obtained from (3.15) with $\varepsilon=0.1, n=1$ and the correct phase $\theta_{n}$ (so that the numerical solution matches the solution in the weakly nonlinear regime).

\subsection{Codimension-two bifurcations}

As shown in Fig. 1 and Fig. 2 for the Schnakenberg and Brusselator kinetics, respectively, the stability curves associated with the Hopf mode $n=0$ and the pitchfork mode $n=1$ intersect at a unique codimension-two bifurcation point. In this subsection, we use the weakly nonlinear theory developed in $\S 3.2$ to explore the dynamics of the full model in the vicinity of such a point.

Near a codimension-two bifurcation point, a linear approximation of the intersecting stability curves is obtained by using the leading-order result (2.82) from the weakly nonlinear analysis. This is done by applying Lemma 3.3 to the generic single-mode stability boundary defined in (3.25). This yields,

$$
\beta_{1}=\mathcal{T}\left(H_{1}\right)=\mu_{0}+\mathcal{R}\left(-\frac{\pi}{2}\right) g_{0010}, \quad \beta_{2}=\mathcal{T}\left(H_{2}\right)=\mu_{0}+\mathcal{R}\left(\frac{\pi}{2}\right) \Re\left(g_{1000}\right),
$$

where $\beta_{1}$ and $\beta_{2}$ are, respectively, tangent to the pitchfork and Hopf stability boundaries.

Next, the cubic term coefficients in (3.20) are evaluated numerically for the Brusselator and Schnakenberg models. These results are given in Table 2. The dynamics of the truncated normal form (3.20) are then classified in Table 3 into two distinct cases. In this table, the reader is referred to (3.22) for the definition of $\gamma, \eta$ and $d$.

Table 2: Numerical evaluation of the coefficients $p_{i j}$ in the normal form (3.20) for the codimension-two bifurcation point for the Schnakenberg and Brusselator kinetics. The parameter values are the same as in the caption of either Fig. 1 (Schnakenberg) or Fig. 2 (Brusselator).

\begin{tabular}{|c|c|c|}
\hline & Schnakenberg & Brusselator \\
\hline$\mu_{0}$ & $(4.26,3.10)^{T}$ & $(4.25,3.38)^{T}$ \\
\hline$p_{11}$ & 0.19096 & 1.3146 \\
\hline$p_{12}$ & -1.2752 & 0.87043 \\
\hline$p_{21}$ & -2.3796 & -0.52089 \\
\hline$p_{22}$ & -0.48351 & -0.162 \\
\hline
\end{tabular}

Table 3: Two specific pitchfork-Hopf unfoldings (see Table 7.5.2 in [13])

\begin{tabular}{|c|c|c|}
\hline & Schnakenberg (case VIII) & Brusselator (case VIa) \\
\hline$d$ & -1 & -1 \\
\hline$\gamma$ & $<0$ & $>0$ \\
\hline$\eta$ & $<0$ & $<0$ \\
\hline$d-\gamma \eta$ & $<0$ & $>0$ \\
\hline
\end{tabular}



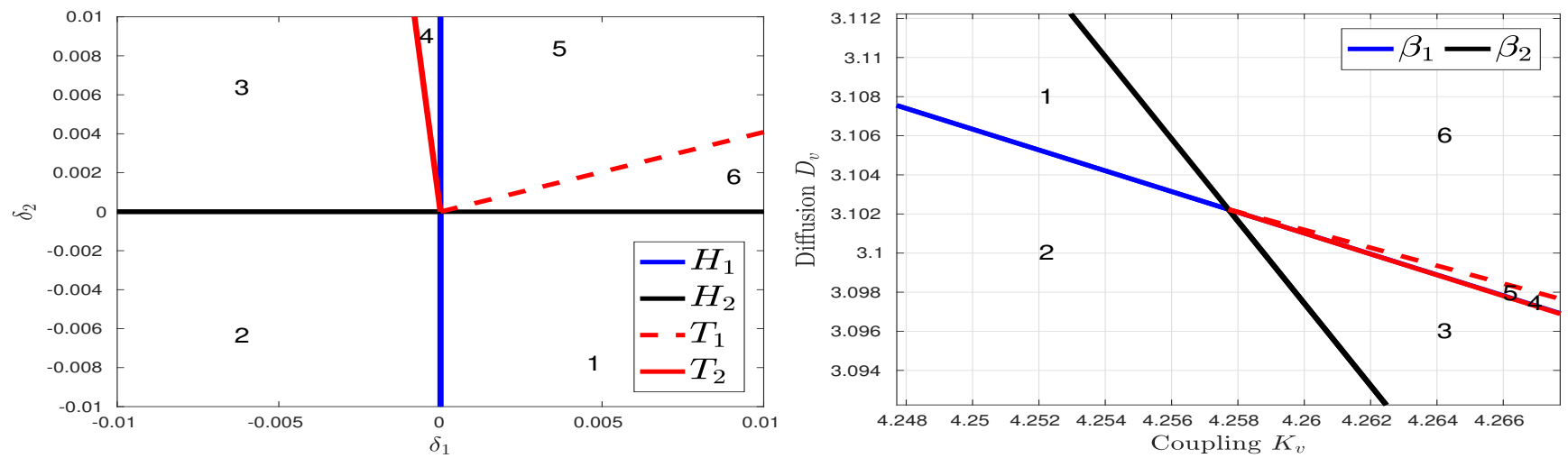

Figure 25: Parametric portraits in the space of generic parameters $\left(\delta_{1}, \delta_{2}\right)$ (left panel) and original bifurcation parameters $\left(K_{v}, D_{v}\right)$ (right panel) for the Schnakenberg kinetics with parameter values as in Fig. 1. In the left panel, the line $H_{1}$ is the vertical $\delta_{2}$-axis (blue), while the line $H_{2}$ is the horizontal $\delta_{1}$-axis (black). The semi-infinite lines $T_{1}$ and $T_{2}$, respectively, correspond to the red dashed and full lines. Application of the map (3.28) to the curves in the left panel yields the curves in the right panel. In particular, the lines $H_{1,2}$ are mapped to $\beta_{1,2}$ (see (4.7)), which are tangent to the pitchfork and Hopf stability boundaries.
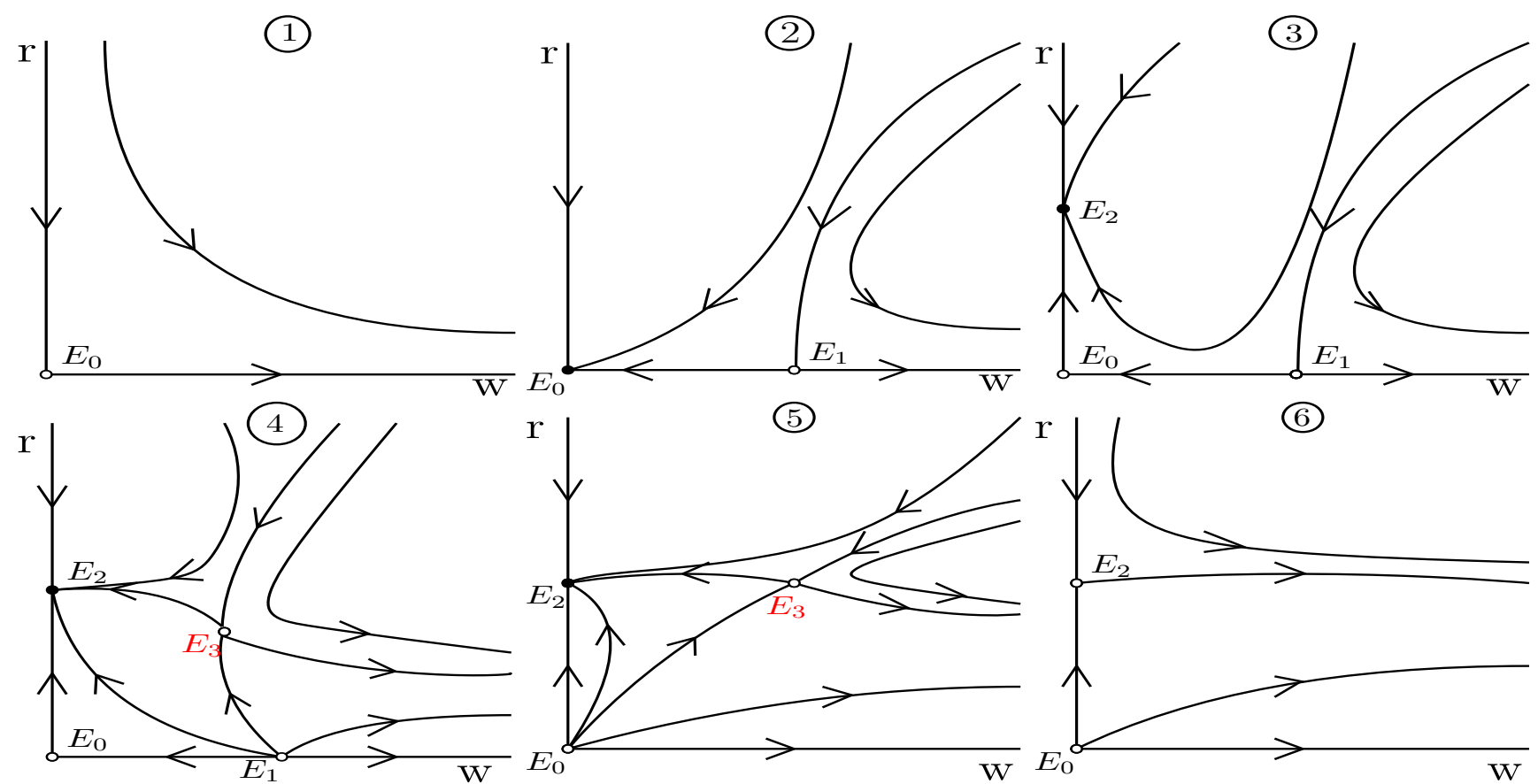

Figure 26: Phase diagrams of the coupled system of amplitude equations (3.21) with unfolding of type VIII (see [13]). The mixed-mode equilibrium $E_{3}$ from panels 4 and 5 is a hyperbolic saddle. 
From Table 3, and based on $\S 4.2$, it follows that the case involving the Schnakenberg kinetics is simple, whereas for the Brusselator kinetics the mixed-mode equilibrium $E_{3}$ undergoes a Hopf bifurcation that is degenerate in the truncated cubic normal form. In this more difficult case, it is an open problem to calculate the fifth-order term in the normal form which, if nonzero, would eliminate this degeneracy.

Parametric portraits for the simple case are given in Fig. 25. The four lines $H_{1}, H_{2}, T_{1}$, and $T_{2}$ divide the $\left(\delta_{1}, \delta_{2}\right)$ parameter plane into six open regions, for which the corresponding phase portraits are shown in Fig. 26. In region 1 , there is a unique unstable equilibrium $E_{0}$. Stability is gained when crossing into region 2, which also generates an unstable equilibrium $E_{2}$. When entering region 3 from region 2 , a stable equilibrium $\left(E_{2}\right)$ bifurcates from the origin while $E_{0}$ becomes unstable again and $E_{1}$ remains unstable. From regions 3 to 4 , the mixed-mode equilibrium $E_{3}$ bifurcates from $E_{1}$. Next, entering region 5 from region 4 causes $E_{1}$ to vanish. When finally crossing the $T_{1}$ line into region 6 , the mixed-mode equilibrium collapses with the single mode equilibrium $E_{2}$, causing $E_{2}$ to lose stability. Moreover, when it exists in regions 4 and 5 , the mixed-mode $E_{3}$ is a hyperbolic saddle whose stable manifold forms the boundary between the basin of attraction of the stable equilibrium $E_{2}$ and some unknown dynamics with large $w$ amplitude.

Restoring the angular variable to (3.20), some equilibria must be interpreted differently. In Fig. 26, the phase portraits may be viewed with the $r$-axis rotating around the $w$-axis. Hence, both $E_{2}$ and $E_{3}$ now correspond to limit cycles, with their stability properties remaining the same. The equilibria $E_{0}$ and $E_{1}$ each remain steady states of the system. The line $H_{2}$ becomes a supercritical Hopf bifurcation, $H_{1}$ remains a subcritical pitchfork bifurcation, while $T_{1}$ and $T_{2}$ each remain a mixed-mode bifurcation.

Since for this simple case the nondegeneracy conditions are satisfied, the stability results associated with the normal form can be interpreted in the context of the bulk-surface PDE model. The origin $E_{0}$ becomes the base state (2.3), $E_{1}$ corresponds to an unstable Turing-type pattern of the first circular harmonic, and $E_{2}$ to radially symmetric nonlinear oscillations. The mixed-mode $E_{3}$ corresponds to nonlinear oscillations around a spatially inhomogeneous equilibrium, which is a type of breather solution. When mapped to the parameter space defined by $K_{v}$ and $D_{v}$, its area of existence becomes fairly narrow (see regions 4 and 5 in the right panel of Fig. 25). Because the mixed-mode solution possesses the stability property of a saddle, bistability between a radially symmetric periodic solution and a large amplitude Turing pattern is expected in this region.

We remark that another equilibrium corresponding to a stable Turing pattern state must also exist because of the dissipative nature of the system, which prevents the solution from becoming unbounded. In $\S 4$.1, numerical evidence for the existence of such a large amplitude stable Turing pattern near a subcritical pitchfork bifurcation was shown for both the Schnakenberg and Brusselator kinetics.

Although it is expected to be unstable, a numerical simulation starting very near the mixed-mode solution should stay near it for some time before drifting away exponentially. PDE simulation results are presented in Fig. 27 for parameter values taken in region 5 and with the initial condition corresponding to $E_{3}$. Good agreement between the weakly nonlinear and numerically computed mixed-mode PDE membrane-bound patterns is shown in the left panel of Fig. 28. This agreement is expected for early simulation time. In the right panel of Fig. 28, we observe that the difference between the PDE numerical solution and the asymptotic mixed-mode solution in the weakly nonlinear regime grows in time.

As time increases, our full numerical results show a transition toward a spatially homogeneous periodic solution. However, in this parameter region, bistability is expected and a different initial condition may lead, instead, to a spatially inhomogeneous equilibrium. Fig. 29 presents two simulation outcomes performed in region 5 , where different initial conditions have led to either a spatially homogeneous periodic solution or a stable Turing pattern.
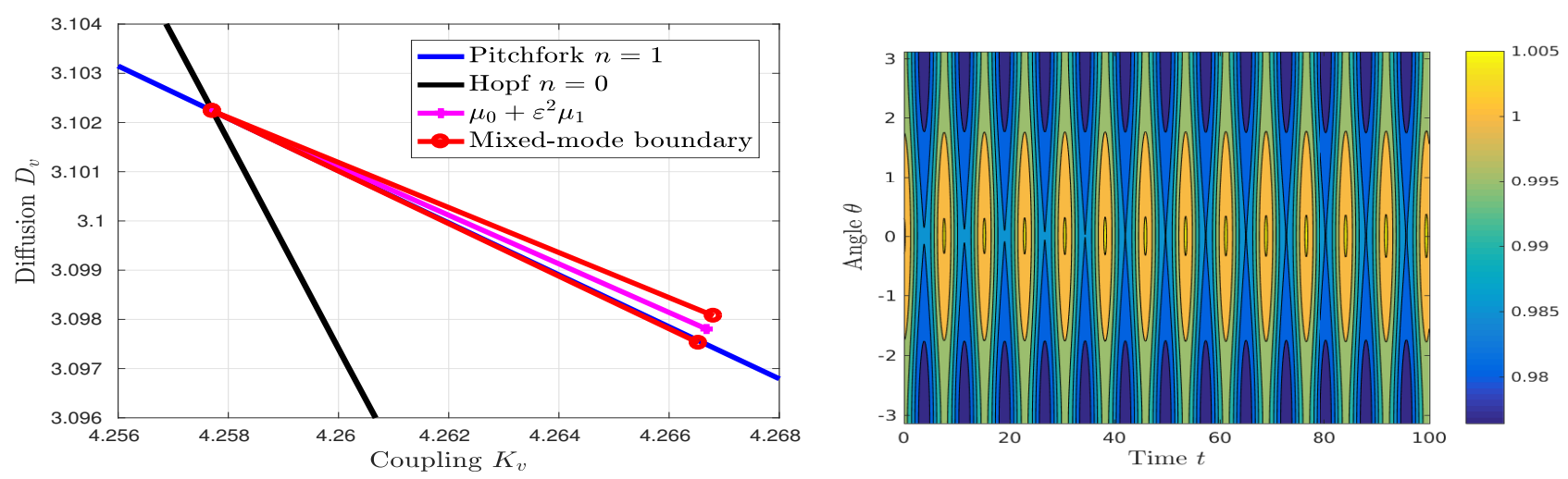

Figure 27: Interaction of a supercritical Hopf and subcritical pitchfork bifurcations for the Schnakenberg kinetics. The simulation corresponds to region 5 (left panel). Right panel: a space-time contour plot of the membrane-bound activator species $u(\theta, t)$, showing oscillations around a spatial pattern. Equation (3.30) with $\varepsilon=0.1, n=1$ and phases $\theta_{0}(0)=\theta_{n}=0$ is used as an initial condition.

Next, we discuss the more intricate case that results from the Brusselator kinetics (see Table 3). Parametric portraits are given in Fig. 30, with corresponding phase diagrams provided in Fig. 31. The regions 1, 2, 3, and 7 yield the same phase diagrams as for the simple case analyzed above. Here, region 7 of Fig. 30 corresponds to region 6 of Fig. 25 . When crossing the line $T_{1}$ from regions 3 to $4, E_{3}$ bifurcates from $E_{2}$, with $E_{2}$ losing stability. In region $4, E_{3}$ is a stable focus while $E_{0}, E_{1}$, and $E_{2}$ are all unstable. On the line $C$, the equilibrium $E_{3}$ undergoes a Hopf bifurcation within the 

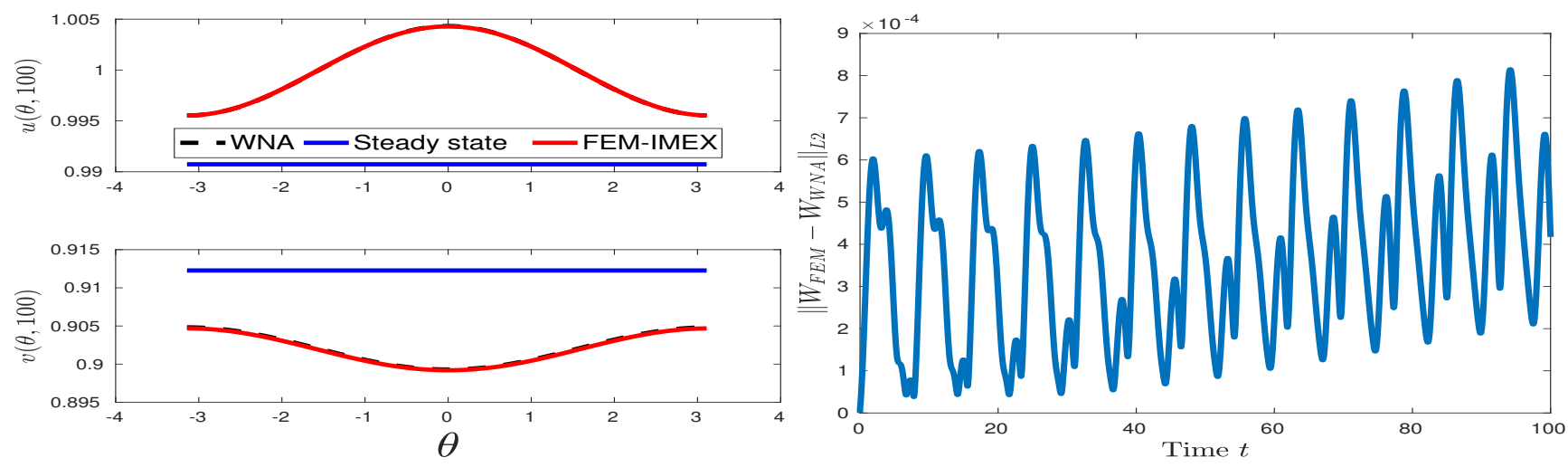

Figure 28: Same simulation as in Fig. 27. Left panel: membrane-bound PDE numerically computed solution (red curve) and the nearly coinciding weakly nonlinear solution (black dashed curve) at time $t=100$. Right panel: difference between these two solutions plotted versus time using the $L^{2}$ norm.
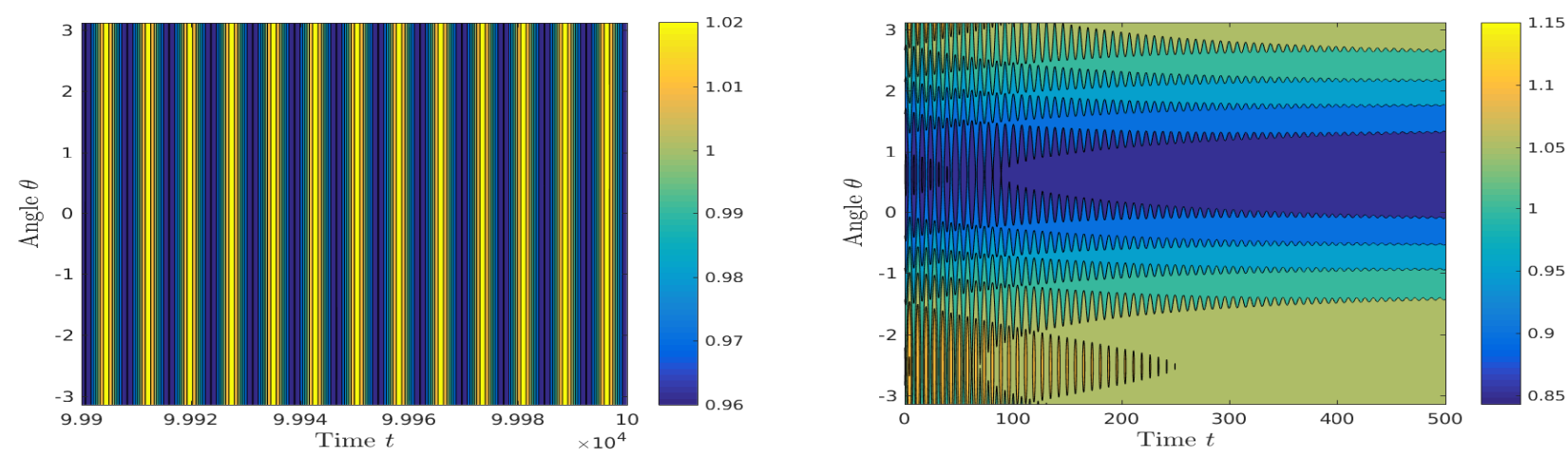

Figure 29: Bistability between a spatially homogeneous periodic solution (left panel) and a stable Turing pattern (right panel) for the Schnakenberg kinetics, with bifurcation parameters taken from region 5 with $\varepsilon=0.1$. Left panel: the initial condition is the mixed-mode solution in the weakly nonlinear regime, with the space-time contour plot showing the long-time oscillatory dynamics of the simulation in the right panel of Fig. 27. Right panel: the initial condition is the base state solution slightly perturbed with the critical eigenvectors of the Jacobian of the spatially discretized system. Notice here that the oscillations become extinguished as time increases. 
truncated system of amplitude equations (3.21). Because only cubic terms are included, the bifurcation is degenerate and the family of limit cycles persist only on the line itself. Also, for this threshold value there is a heteroclinic connection between the two single mode equilibria. In region 5 , the four equilibria are unstable. Finally, between regions 5 and 7 , the successive crossing of the lines $T_{2}$ and $H_{2}$ causes the mixed-mode equilibrium to collapse on $E_{1}$, after which $E_{1}$ collapses at the origin.

Restoring the angular variable to the truncated system of amplitude equations, we expect torus (Neimarck-Sacker) bifurcations for parameter values on a curve tangent to $C$ and an exponentially thin $\left(\right.$ as $\left.\left(\delta_{1}, \delta_{2}\right) \rightarrow 0\right)$ region of parameters near $C$ corresponding to some kind of chaotic behaviour (see [17] and [27]). The possibility of such intricate dynamics is interesting, but it seems likely to be confined to an extremely small region of parameter space that would be virtually undetectable in PDE simulations.
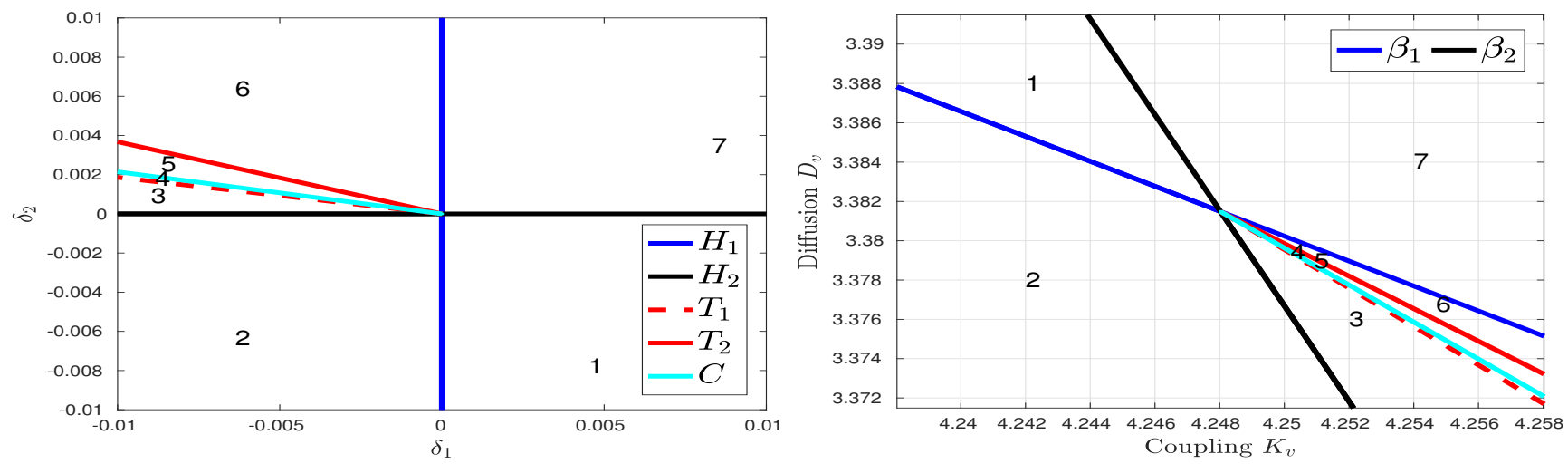

Figure 30: Parametric portraits in the space of generic parameters $\left(\delta_{1}, \delta_{2}\right)$ (left panel) and original bifurcation parameters $\left(K_{v}, D_{v}\right)$ (right panel) with Brusselator kinetics. The lines $H_{1,2}$ and $T_{1,2}$ are described in the caption of Fig. 25 , with the additional line $C$ (equation (3.27)) in cyan color in the left panel. Applying the affine transformation defined in Lemma 3.3 yields the plot in the right panel.
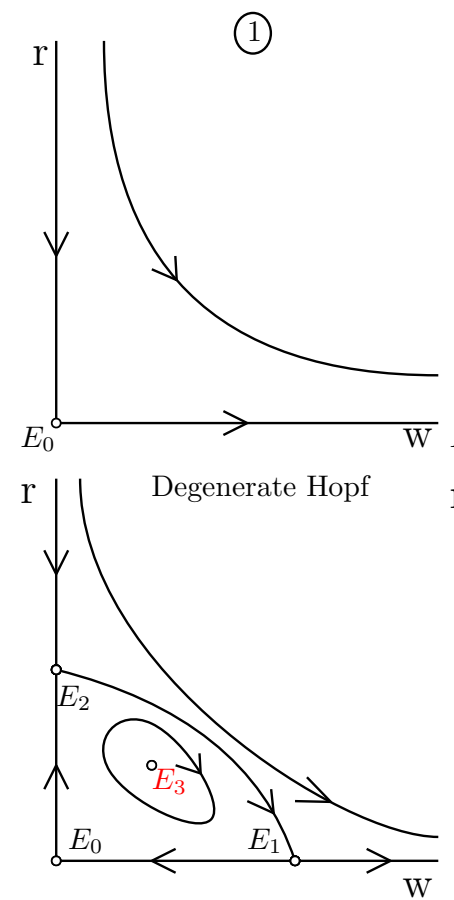
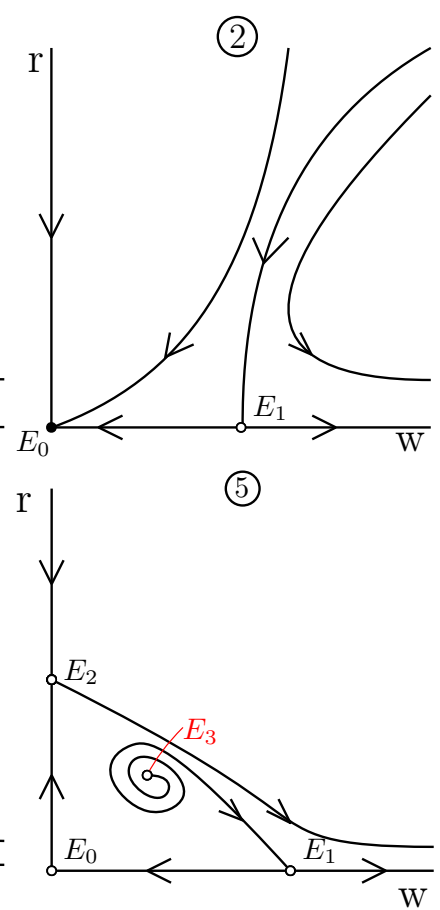
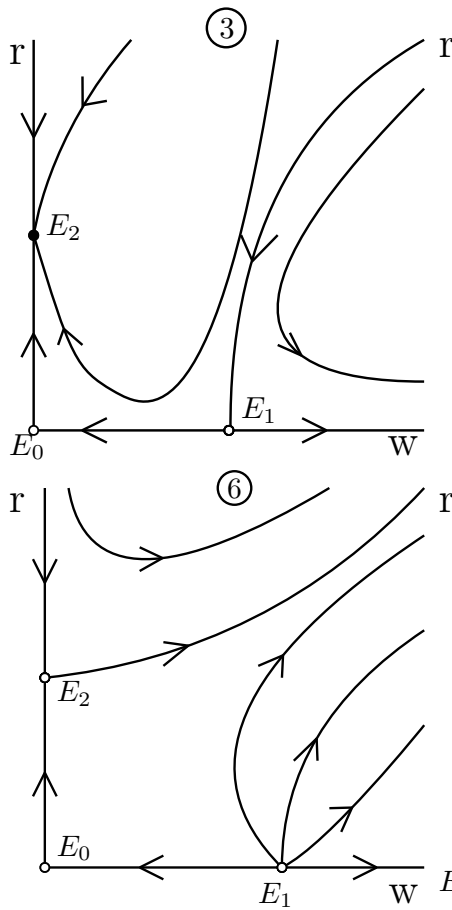

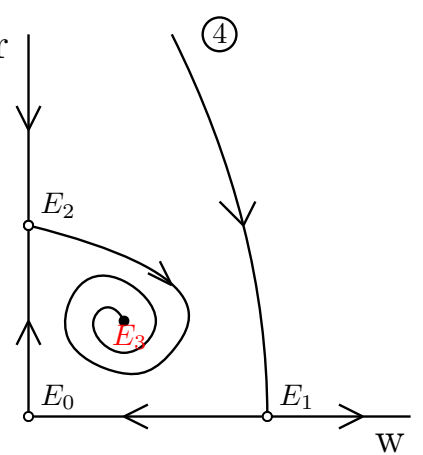

(7)

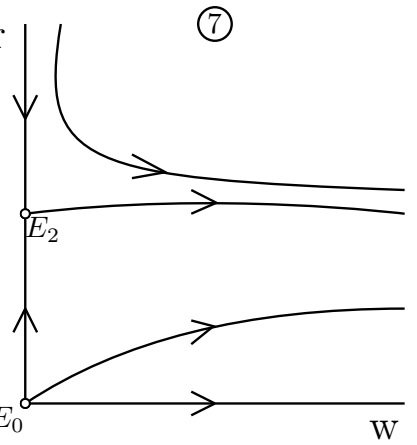

Figure 31: Phase diagrams of the truncated system of amplitude equations (3.21) with unfolding of type VIa (see Fig. 7.5.5 of [13]). The phase diagram corresponding to the line $C$ in Fig. 30 is structurally unstable, i.e. the retention of generic higher-order terms will remove the degeneracy of the Hopf bifurcation and introduce a heteroclinic orbit (see [13] and [17] for more details).

In order to remove the degeneracy of the Hopf bifurcation, higher-order terms should be added to the normal form. Since this challenging computation, starting from our coupled bulk-surface PDE model, is left as an open problem, some bifurcation results from regions 4, 5 and on the line $C$ in Fig. 30 cannot be transferred to the original system. It is nevertheless possible to investigate numerically the breather-type solutions within this narrow parameter regime (when considering the space defined by the original bifurcation parameters). Fig. 32 shows simulation results for short times, and for parameter values taken in region 5 , where the mixed-mode solution is expected to be unstable.

The right panel of Fig. 33 shows that the distance between the numerical solution and the solution in the weakly 

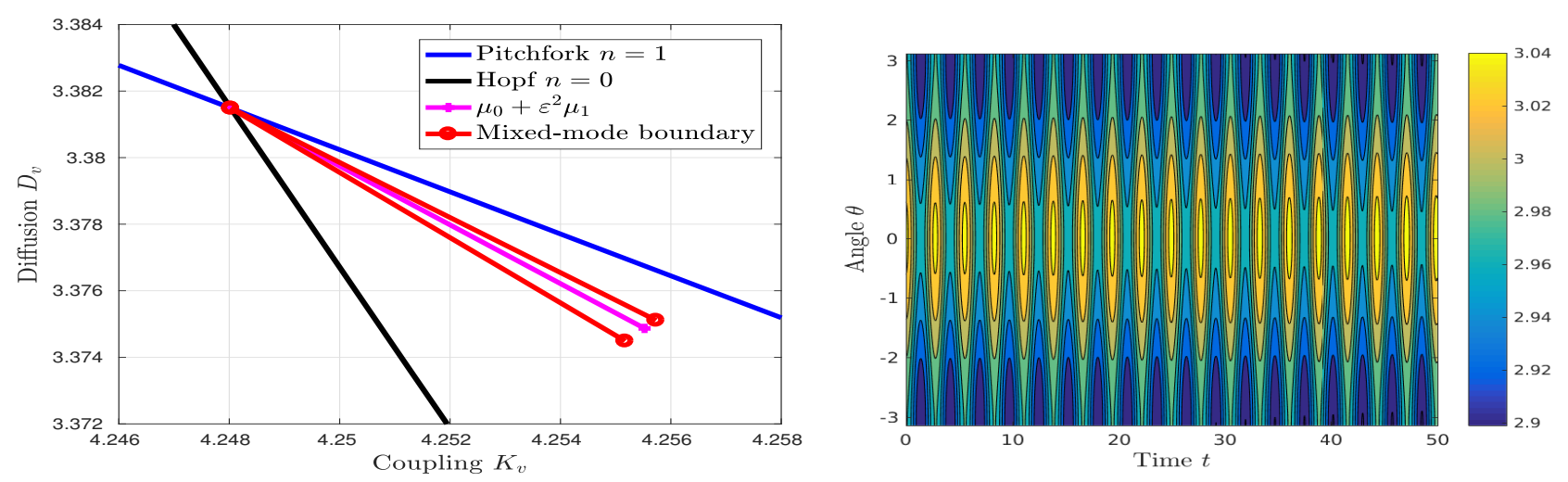

Figure 32: Interaction of a supercritical Hopf and subcritical pitchfork bifurcations for Brusselator kinetics with $\varepsilon=0.1$. The simulation corresponds to region 5 (see left panel). Right panel: a space-time contour plot of the membrane-bound activator species $u(\theta, t)$, which exhibits oscillations around some spatial pattern. The initial condition is the mixed-mode solution in the weakly nonlinear regime.
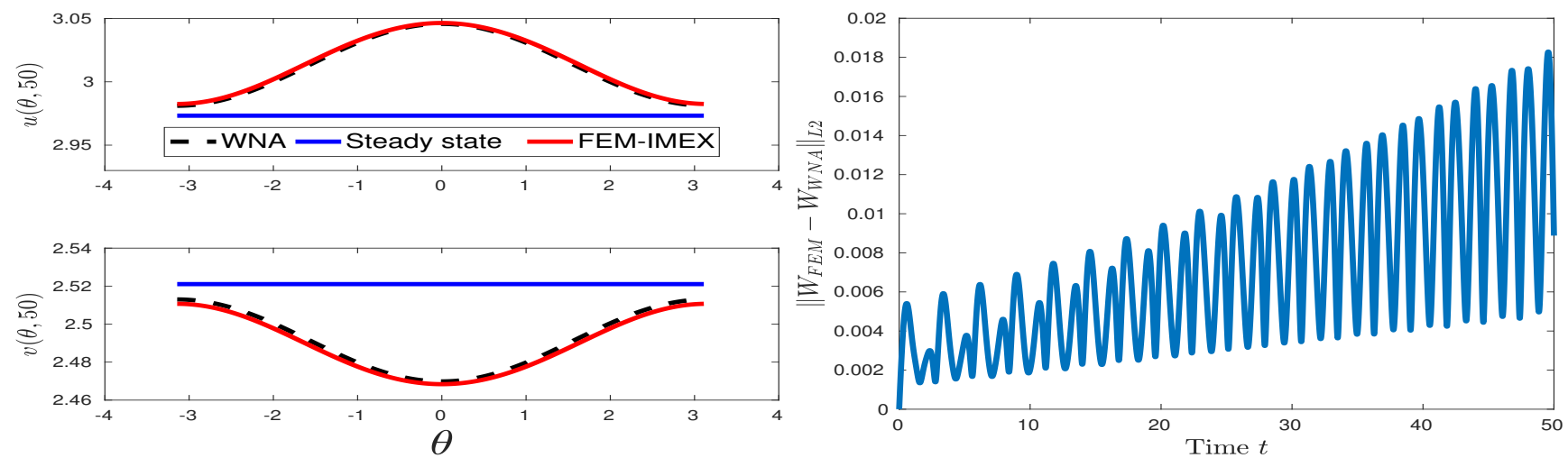

Figure 33: Same simulation as in Fig. 32 for the Brusselator kinetics, with $\varepsilon=0.1$. Left panel: the membrane-bound numerical (red curve) and the nearly coinciding weakly nonlinear (black dashed curve) solutions at time $t=50$. Right panel: difference between these two solutions plotted over time using the $L^{2}$ norm. 
nonlinear regime grows over time. However, in contrast to the previous case with the Schnakenberg kinetics, the long time integration in Fig. 34 clearly reveals a transition towards a spatially inhomogeneous steady state and the absence of bistability with the spatially homogeneous periodic solution. This is consistent with the phase diagram for parameters in region 5 in Fig. 30 and Fig. 31.
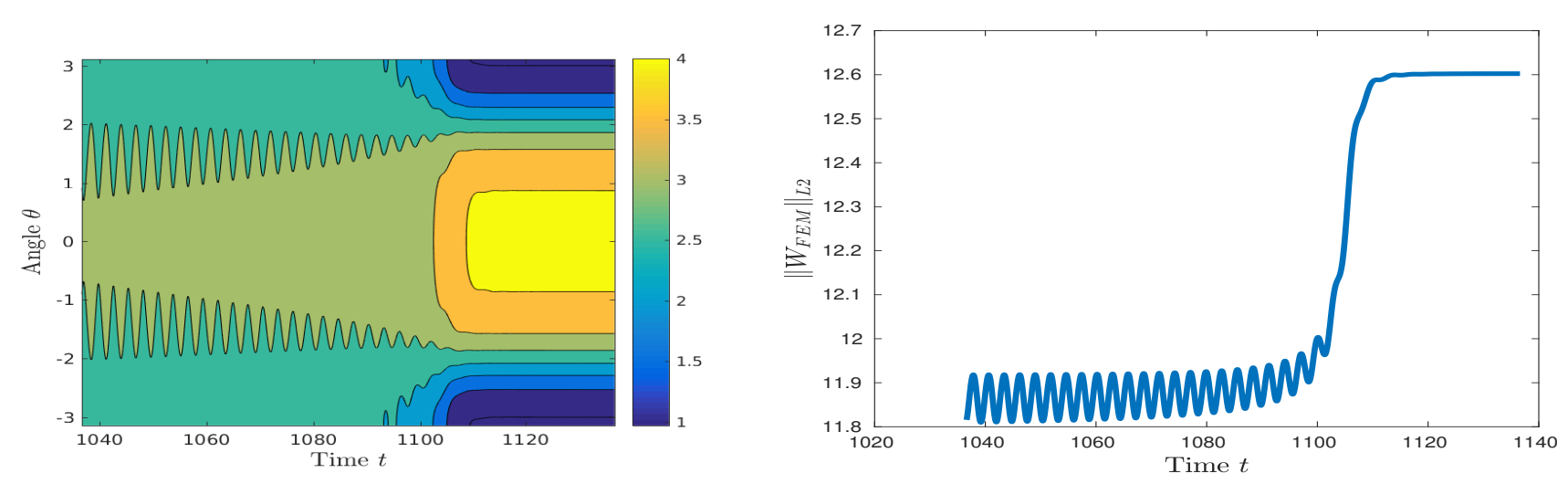

Figure 34: Transition between the mixed-mode solution and a stable Turing pattern for the Brusselator kinetics for bifurcation parameters taken from region 5 of Fig. 31 with $\varepsilon=0.1$. Left panel: a space-time contour plot. Right panel: plot of the $L^{2}$ norm of the numerical solution. The initial transient has been removed for both plots.

Finally, we remark that when applied to systems of PDEs, the normal form analysis of codimension-two bifurcations must be interpreted with care, especially when degenerate local bifurcations occur in the system of amplitude equations [28]. Moreover, the parameter regime and phase space ranges where the conclusions hold can be fairly narrow, making it very difficult for PDE direct numerical simulations to reproduce delicate dynamical behaviors that occur in the ODE amplitude equations.

\section{Global dynamics via full numerics: rotating waves}

In this section, full numerical simulations are used to briefly explore novel dynamical behaviors in the highly nonlinear regime, away from bifurcation points, that are due to the bulk-surface coupling. For the Brusselator reaction kinetics, we study the formation of rotating waves and show that they arise when a nontrivial spatial mode undergoes a Hopf bifurcation. Allowing for different adsorption and desorption rates for each species seems to be a key condition behind the formation of such waves.

We consider here a modified coupled bulk-surface model for which the rates of adsorption and desorption are different for each species. Let $r_{a}$ and $p_{a}$ be the activator and inhibitor rates of adsorption. Similarly, let $r_{d}$ and $p_{d}$ be the desorption rates. Then, the boundary conditions in (1.3) are reformulated as

$$
\left.D_{u} \partial_{r} U\right|_{r=R}=r_{d} u-\left.r_{a} U\right|_{r=R},\left.\quad D_{v} \partial_{r} V\right|_{r=R}=p_{d} v-\left.p_{a} V\right|_{r=R} .
$$

Similar boundary conditions are considered in [18] and [20]. These new boundary conditions modify the dynamics on the surface, so that (1.4) is replaced by

$$
\partial_{t} u=\frac{d_{u}}{R^{2}} \partial_{\theta \theta} u-r_{d} u+\left.r_{a} U\right|_{r=R}+f(u, v), \quad \partial_{t} v=\frac{d_{v}}{R^{2}} \partial_{\theta \theta} v-p_{d} v+\left.p_{a} V\right|_{r=R}+g(u, v) .
$$

After calculating the radially symmetric base state for this modified bulk-surface model, a linear stability analysis readily provides a transcendental equation for the growth rate $\lambda$ associated with the circular harmonic of mode $n$. In place of (2.10), the growth rates are roots of $F_{n}(\lambda)=0$, where

$$
F_{n}(\lambda)=\left(\lambda-f_{u}^{e}+\frac{r_{d}}{1+\frac{r_{a} I_{n}\left(\Omega_{u} R\right)}{D_{u} \Omega_{u} I_{n}^{\prime}\left(\Omega_{u} R\right)}}+\frac{n^{2} d_{u}}{R^{2}}\right)\left(\lambda-g_{v}^{e}+\frac{p_{d}}{1+\frac{p_{a} I_{n}\left(\Omega_{v} R\right)}{D_{v} \Omega_{v} I_{n}^{\prime}\left(\Omega_{v} R\right)}}+\frac{n^{2} d_{v}}{R^{2}}\right)-f_{v}^{e} g_{u}^{e}
$$

Following a remark from [18] on the conditions underlying the emergence of traveling waves, we restrict the parameter space by setting the diffusion coefficients to be equal for both species. More specifically, the following set of parameters is considered:

$$
R=1, D_{u}=D_{v}=1, \sigma_{u}=\sigma_{v}=0.5, d_{u}=d_{v}=0.5, r_{a}=0.1, r_{d}=1, p_{a}=1, p_{d}=0.1, a=3 .
$$

By allowing the Brusselator kinetic parameter $b$ in (1.6) to be free in equation (5.3), in Fig. 35 we show numerically that the system undergoes a series of Hopf bifurcations, each of which is associated with a spatial mode $n$. Notice in the left panel of Fig. 35 that the trivial mode is the first to lose stability. Hence, we expect the waves to coexist with radially symmetric oscillations in the fully nonlinear regime. 
For $b=8$, full PDE numerical computations of this modified bulk-surface model reveal three distinct types of temporally oscillatory solutions depending on the initial data. A clockwise rotating wave is shown in Fig. 36, an anticlockwise rotating wave is shown in Fig. 37, and finally a radially symmetric oscillatory solution is shown in Fig. 38 . For each case, appropriate initial conditions favoring a particular mode have led to the desired dynamics. We have also tried to compute a standing wave by stimulating the modes $n= \pm 1$, but our numerical results suggest such a solution to be unstable.
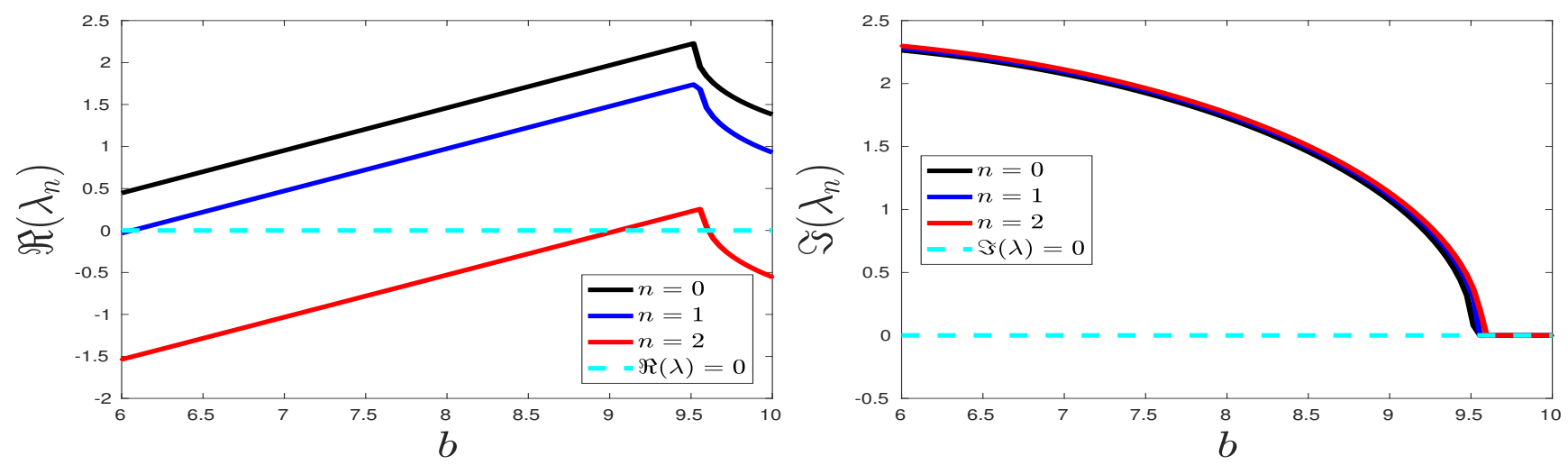

Figure 35: Real (left panel) and imaginary (right panel) parts of the most unstable eigenvalues, computed from (5.3), for the mode $n=0,1,2$ as the kinetic parameter $b$ increases. The parameters are given in (5.4).
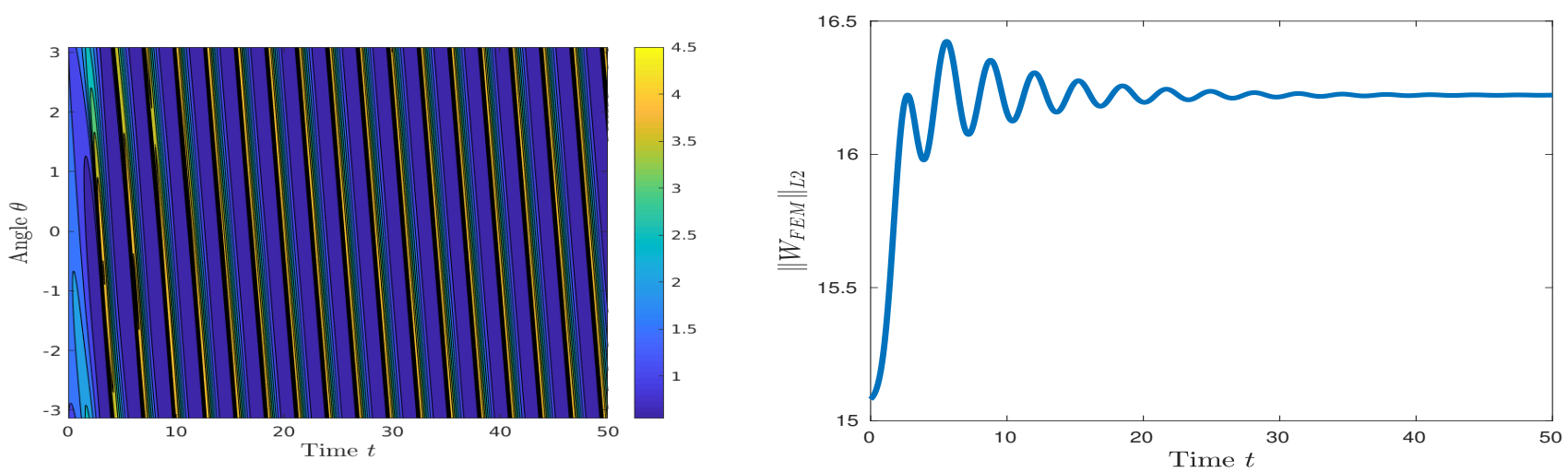

Figure 36: Clockwise rotating waves for the Brusselator kinetics (1.6) with $b=8$ and for the parameter set (5.4). The initial condition corresponds to a perturbation of the base state solution favoring the mode $n=1$. The left panel shows a space-time contour plot of the membrane-bound activator species. In the right panel, the $L^{2}$ norm of the solution converges to some equilibrium values after an initial oscillatory transient.

These numerical results give only a glimpse of novel global solution structures for coupled bulk-surface models that can occur away from bifurcation points. A rigorous analysis of the existence of such rotating traveling waves, including a precise determination of the parameter space involving the adsorption and desorption rates where they occur, is beyond the scope of this paper.

\section{Discussion}

On a two-dimensional circular domain, we have introduced and analyzed a class of coupled bulk-surface reaction-diffusion models for which a passive diffusion process occurring in the interior bulk domain is linearly coupled to a nonlinear reaction-diffusion process on the domain boundary. In $\S 2$, a multiple time-scale approach was employed to systematically derive amplitude equations near three different instabilities: the Hopf, the pitchfork (or Turing), and the pitchfork-Hopf bifurcations. An interesting feature of our development of the weakly nonlinear theory of pattern formation for coupled bulk-surface PDE models was the analysis of the spectral problem for the linearization, which involved the eigenvalue parameter appearing in the boundary conditions. In $\S 3$, we used the normal form equations to determine the stability of bifurcating branches in the weakly nonlinear regime. The theory was illustrated using the classical Schnakenberg and Brusselator kinetics in $\S 4$, where good agreement between numerical and analytical solutions was observed. Our hybrid analytical-numerical approach has shown that the linear coupling of a diffusive bulk to an active membrane can lead to either oscillatory dynamics or pattern formation. Finally, the formation of rotating waves is explored through numerical simulations in $\S 5$.

Several open problems related to coupled bulk-surface reaction-diffusion systems warrant further investigation. One challenge concerns the computation of global bifurcating branches, a task amenable to numerical bifurcation analysis. The classical software AUTO [6] has been successfully applied to the reduced 1-D radially symmetric model with angular 

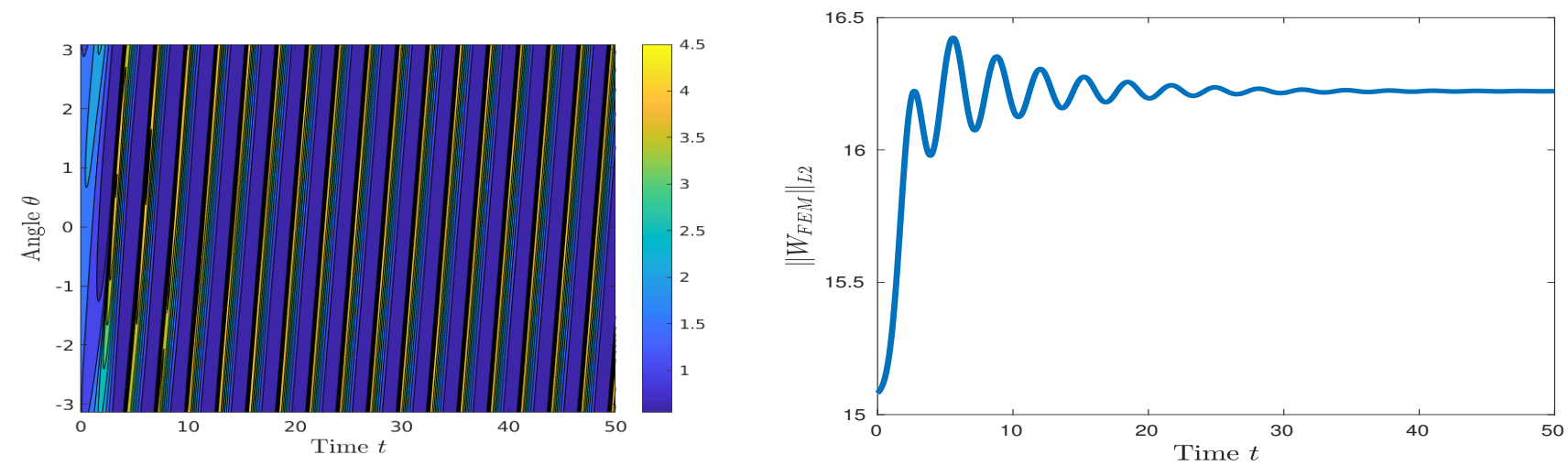

Figure 37: Anti-clockwise rotating waves with the Brusselator kinetics (1.6) and the same parameter values as in Fig. 36. The initial condition corresponds to a perturbation of the base state solution favoring the mode $n=-1$. The left panel shows a space-time contour plot of the membrane-bound activator species. In the right panel, we see the $L^{2}$ norm of the solution converging to some equilibrium values in a similar fashion as for the clockwise waves.
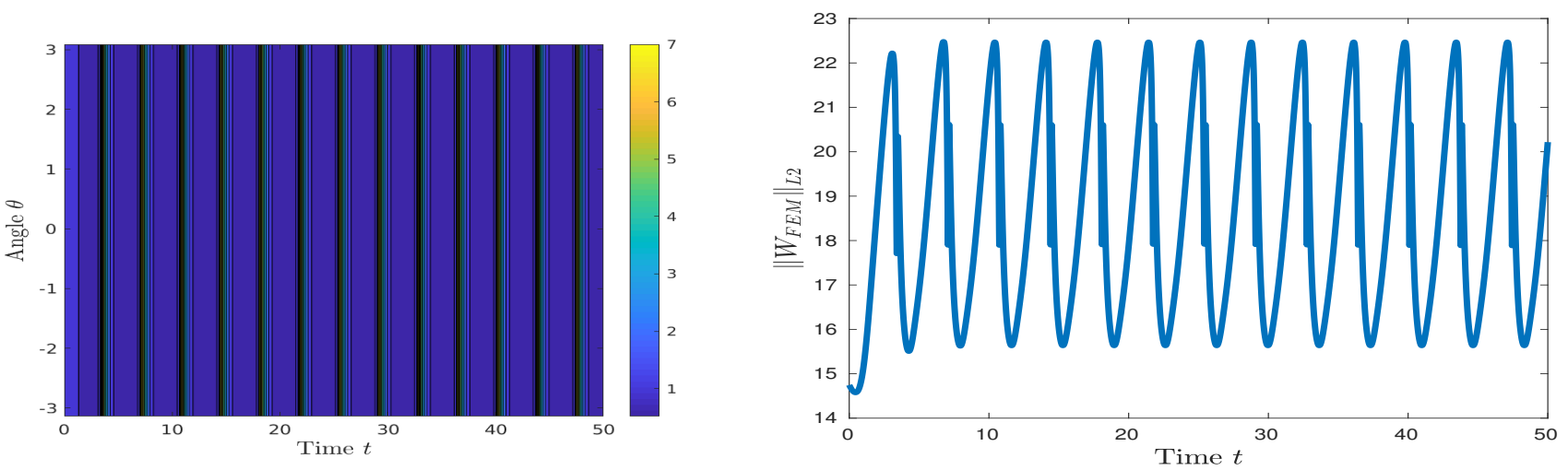

Figure 38: Radially symmetric oscillations with the Brusselator kinetics and the same parameter values as in Figs. 36-37. The initial condition corresponds to a perturbation of the base state solution favoring the trivial mode $(n=0)$. The left panel shows a space-time contour plot of the membrane-bound activator species, which clearly exhibits spatially uniform oscillations. In the right panel, the solution in $L^{2}$ norm undergoes sustained oscillations. 
invariance (in the context of Hopf bifurcations), but it cannot handle implicit systems of differential equations such as those obtained after discretizing the full 2-D model using finite elements (see Appendix A for details). A promising alternative to AUTO that has been explored is the software package COCO (cf. [4]). The Equilibrium Point toolbox from COCO, combined with the PDE toolbox from MATLAB [21], has been used to compute base state solution families of the full model. Successive mesh refinement has revealed quadratic convergence between the bifurcation points predicted by the weakly nonlinear theory and as detected by COCO. However, because of rotational symmetries, we have been unable to numerically branch off at a pitchfork bifurcation point. These bifurcations are characterized by the crossing of two nearly identical eigenvalues (spatial discretization causes some loss of symmetry) through the origin, and thus there are two critical eigenvectors at the branch point. Further work in this direction is needed in order to numerically resolve the bifurcating branch arising from this rather degenerate bifurcation point.

The weakly nonlinear analysis carried out in this work has revealed a rich bifurcation structure consisting of both subcritical and supercritical codimension-one bifurcations, as well as codimension-two pitchfork-Hopf bifurcations. On two occasions, the cubic normal forms derived in $\S 2$ were not sufficient to capture the dynamical behavior of the original system up to topological equivalence. The first situation, discussed in $\$ 4.1 .1$ for the Brusselator membrane kinetics, concerns the transition from a supercritical to a subcritical Hopf bifurcation. There, parameter values at which the bifurcation becomes a degenerate Hopf (Bautin) bifurcation were found. For the same kinetics, the classification of codimension-two bifurcations in $\S 4.2$ has also revealed some degeneracy in the phase portraits of the truncated system of amplitude equations, which resulted from the mixed-mode equilibrium undergoing a Hopf bifurcation. For those two cases, the computation of an additional (nonzero) term is needed to fully resolve the degeneracy in the normal forms. Further details on this lengthy computation for simpler ODE models can be found in [17] and [13].

Through numerical simulations, our work has revealed the existence of clockwise and anti-clockwise rotating waves coexisting with radially symmetric oscillations. Hence, an open question amenable to a more rigorous PDE theory approach consists of proving the existence and the stability of the waves. Key to this problem is the appropriate reformulation of the model into a moving coordinate frame. Also, it would be worthwhile to precisely delineate the region in the adsorption-desorption parameter space where rotating traveling waves can occur.

With their confined geometry, and because of the clear distinction between the dynamics in the domain and on its boundary, coupled bulk-surface reaction-diffusion models are ideal for investigating intracellular pattern-forming systems. For instance, a bulk-surface model for the spatio-temporal Min protein patterning within E. Coli was formulated in [14] in a two-dimensional elliptical geometry. To our knowledge, prior studies are often limited to linear stability analysis and full numerical simulations (cf. [18], [20]). An open problem is to extend the weakly nonlinear theory developed in $\S 2$ to some biologically relevant bulk-surface models in more general classes of domains (cylinders, spheres, ellipses).

\section{Appendix A Numerical methods}

In this appendix, the various numerical techniques employed in this paper are briefly explained. We first focus on the finite differences discretization of the model with radial symmetry. Then, we present the finite element discretization of the full bulk-surface reaction-diffusion system. Finally, the specific Implicit-Explicit time-stepping method used for most numerical simulations is discussed.

\section{A.1 Finite differences for the radially symmetric case}

Assuming angular invariance of the coupled bulk-surface system, (1.2) and (1.4) become

$$
\begin{aligned}
& \frac{\partial U}{\partial t}=\frac{D_{u}}{r} \frac{\partial}{\partial r}\left(r \frac{\partial U}{\partial r}\right)-\sigma_{u} U, \quad \frac{\partial V}{\partial t}=\frac{D_{v}}{r} \frac{\partial}{\partial r}\left(r \frac{\partial V}{\partial r}\right)-\sigma_{v} V, \quad 0<r<R, \\
& \frac{d u}{d t}=-K_{u}\left(u-\left.U\right|_{r=R}\right)+f(u, v), \quad \frac{d v}{d t}=-K_{v}\left(v-\left.V\right|_{r=R}\right)+g(u, v) .
\end{aligned}
$$

The coupling between the PDEs in the bulk and the ODEs on the boundary occurs through the same linear Robin-type boundary conditions as given in (1.3).

We let $h=R /(N-1)$ be the step size, where $N$ is the number of mesh points. We then approximate $U_{j}(t) \approx$ $U(h(j-1), t)$ and $V_{j}(t) \approx V(h(j-1), t)$, for $j=1, \ldots, N$. Next, employing the method of lines yields the following system of ODEs for the vector $\boldsymbol{W}=\left(U_{1}, \ldots, U_{N}, V_{1}, \ldots, V_{n}, u, v\right)^{T} \in \mathbb{R}^{2 N+2}$ :

$$
\dot{\boldsymbol{W}}=\mathbb{A} \boldsymbol{W}+\boldsymbol{F}(\boldsymbol{W}) .
$$

Here, $\mathbb{A} \in \mathbb{R}^{(2 N+2) \times(2 N+2)}$ is the block diagonal matrix defined by

$$
\mathbb{A}=\left(\begin{array}{ccc}
D_{u} \mathbb{L}-\sigma_{u} \mathbb{I}-K_{u}\left(\frac{2}{h}+\frac{1}{R}\right) e_{N} e_{N}^{T} & \mathbb{O} & \mathbb{O} \\
\mathbb{O} & D_{v} \mathbb{L}-\sigma_{v} \mathbb{I}-K_{v}\left(\frac{2}{h}+\frac{1}{R}\right) e_{N} e_{N}^{T} & \mathbb{O} \\
\mathbb{O} & \mathbb{O} & \mathbb{O}
\end{array}\right)
$$

where $\mathbb{I} \in \mathbb{R}^{N \times N}$ is the identity matrix, $e_{N}=(0, \ldots, 1)^{T} \in R^{N}$ and each instance of $\mathbb{O}$ is an appropriate matrix of zeros. 
Also, $\mathbb{L} \in \mathbb{R}^{N \times N}$ corresponds to the discrete radially symmetric Laplacian, defined by

$$
\mathbb{L}=\frac{1}{h^{2}}\left(\begin{array}{ccccc}
-4 & 4 & 0 & \ldots & 0 \\
\frac{1}{2} & -2 & \frac{3}{2} & \ldots & 0 \\
\vdots & \ddots & \ddots & \ddots & \vdots \\
0 & \ldots & 1-\frac{1}{2(N-2)} & -2 & 1+\frac{1}{2(N-2)} \\
0 & \ldots & 0 & 2 & -2
\end{array}\right) .
$$

Finally, the nonlinear function $\boldsymbol{F}(\boldsymbol{W}): \mathbb{R}^{2 N+2} \rightarrow \mathbb{R}^{2 N+2}$ is defined by

$$
\boldsymbol{F}(\boldsymbol{W})=\left(\begin{array}{c}
K_{u}\left(\frac{2}{h}+\frac{1}{R}\right) u e_{N} \\
K_{v}\left(\frac{2}{h}+\frac{1}{R}\right) v e_{N} \\
-K_{u}\left(u-U_{N}\right)+f(u, v) \\
-K_{v}\left(v-V_{N}\right)+g(u, v)
\end{array}\right)
$$

\section{A.2 Finite element discretization}

Let $\Omega$ be the two-dimensional circular bulk domain of radius $R$. In order to derive the weak formulation for equation (1.2) at each $t>0$, we multiply it by $\phi \in H^{1}(\Omega)$ and integrate by parts using the boundary conditions (1.3). This yields that

$$
\begin{array}{rlrl}
\int_{\Omega} \phi U_{t} & =K_{u} \int_{\partial \Omega} \phi(u-U)-D_{u} \int_{\Omega} \nabla \phi \cdot \nabla U-\sigma_{u} \int_{\Omega} \phi U, & & \forall \phi \in H^{1}(\Omega), \\
\int_{\Omega} \phi V_{t} & =K_{v} \int_{\partial \Omega} \phi(v-V)-D_{v} \int_{\Omega} \nabla \phi \cdot \nabla V-\sigma_{v} \int_{\Omega} \phi V, & \forall \phi \in H^{1}(\Omega) .
\end{array}
$$

We then define an appropriate mesh on $\Omega$. First, we can parametrize the boundary $\partial \Omega$ by the arc-length as

$$
\partial \Omega=\left\{\boldsymbol{X}(\sigma) \in \mathbb{R}^{2} \mid 0 \leq \sigma<2 \pi R\right\} .
$$

For simplicity, the nodes on the boundary are chosen to be evenly spaced by an arc-length step size of $d \sigma=2 \pi R / N$, where $N$ is the number of nodes on the boundary. Let $N_{\text {total }}$ denote the total number of mesh points in $\Omega$, which includes those on the boundary. A partition can then be defined as follows:

$$
\Delta_{h_{\max }}=\left\{x_{i}=\boldsymbol{X}((i-1) d \sigma) \mid i=1, \ldots, N\right\} \cup\left\{x_{i} \mid\left\|x_{i}\right\|<R \text { for } i=N+1, \ldots, N_{\text {total }}\right\},
$$

where $h_{\max }$ is the maximal distance between two adjacent nodes, defined by

$$
h_{\max }=\max _{j} \min _{i \neq j}\left\|x_{i}-x_{j}\right\| .
$$

In Fig. 39, we plot two different meshes approximating the unit disk given $N=200$ boundary nodes.
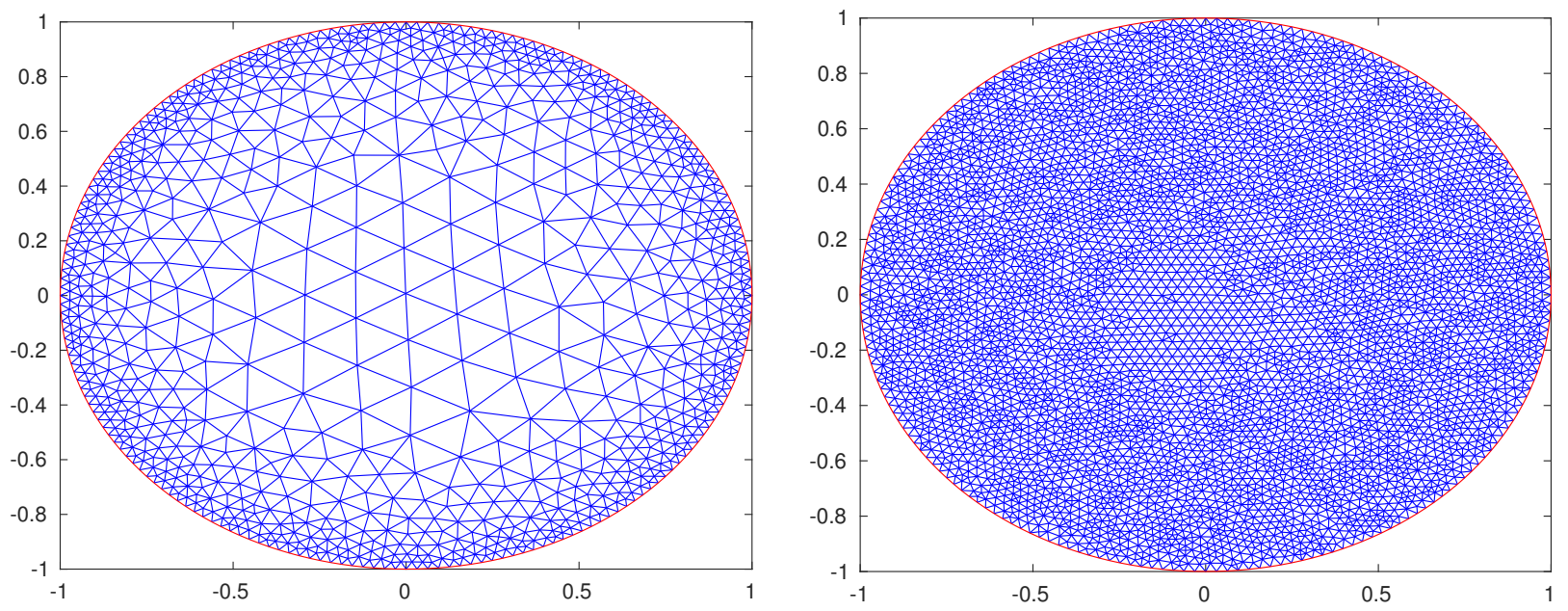

Figure 39: Two different meshes approximating the unit disk for $N=200$, obtained with the PDE Toolbox of MATLAB [21]. In the left panel, the mesh is finer near the boundary than in the center of the bulk domain. In contrast, in the right panel, we have set $h_{\max } \equiv d \sigma$.

Now, let $S_{h_{\max }}(\Omega)$ be the space of piecewise linear functions defined on the mesh $\Delta_{h_{\max }}$ and define a basis $\left\{\phi_{i}\right\}$ such that any element $U_{h_{\max }} \in S_{h_{\max }}(\Omega)$ can be uniquely written as

$$
U_{h_{\max }}=\sum_{i=1}^{N_{\text {total }}} U_{i}(x) \phi(x) .
$$


Hence, the weak formulation (A.7) is approximated as

$$
\begin{aligned}
& \int_{\Omega} \phi_{i} U_{t}=K_{u} \int_{\partial \Omega} \phi_{i}(u-U)-D_{u} \int_{\Omega} \nabla \phi_{i} \cdot \nabla U-\sigma_{u} \int_{\Omega} \phi_{i} U, \quad \text { for } i=1, \ldots, N_{\text {total }}, \\
& \int_{\Omega} \phi_{i} V_{t}=K_{v} \int_{\partial \Omega} \phi_{i}(v-V)-D_{v} \int_{\Omega} \nabla \phi_{i} \cdot \nabla V-\sigma_{v} \int_{\Omega} \phi_{i} V, \quad \text { for } i=1, \ldots, N_{\text {total }} .
\end{aligned}
$$

Assuming that the first basis functions $\phi_{i}(x)$ for $i=1, \ldots, N$ form a piecewise linear basis for the polygonal approximation of the boundary, we can approximate the bulk $U, V$ and surface $u, v$ concentrations by

$$
U_{h_{\max }}=\sum_{i=1}^{N_{\text {total }}} U_{i}(t) \phi_{i}(x), \quad V_{h_{\max }}=\sum_{i=1}^{N_{\text {total }}} V_{i}(t) \phi_{i}(x), \quad u_{h_{\max }}=\sum_{i=1}^{N} u_{i}(t) \phi_{i}(x), \quad v_{h_{\max }}=\sum_{i=1}^{N} v_{i}(t) \phi_{i}(x) .
$$

Then, substituting (A.13) into (A.12) we obtain the following linear system of ODEs:

$$
\begin{aligned}
& \mathbb{M} \dot{\boldsymbol{U}}=-\left(K_{u} \mathbb{Q}+D_{u} \mathbb{K}+\sigma_{u} \mathbb{M}\right) \boldsymbol{U}+K_{u} \mathbb{Q B}^{T} \boldsymbol{u} \\
& \mathbb{M} \dot{\boldsymbol{V}}=-\left(K_{v} \mathbb{Q}+D_{v} \mathbb{K}+\sigma_{v} \mathbb{M}\right) \boldsymbol{V}+K_{v} \mathbb{Q B B}^{T} \boldsymbol{v}
\end{aligned}
$$

where the vectors $\boldsymbol{U}, \boldsymbol{V} \in \mathbb{R}^{N_{\text {total }}}$ and $\boldsymbol{u}, \boldsymbol{v} \in \mathbb{R}^{N}$ are defined by

$$
\boldsymbol{U}=\left(\begin{array}{c}
U_{1}(t) \\
\vdots \\
U_{N_{\text {total }}(t)}
\end{array}\right), \quad \boldsymbol{V}=\left(\begin{array}{c}
V_{1}(t) \\
\vdots \\
V_{N_{\text {total }}}(t)
\end{array}\right), \quad \boldsymbol{u}=\left(\begin{array}{c}
u_{1}(t) \\
\vdots \\
u_{N}(t)
\end{array}\right), \quad \boldsymbol{v}=\left(\begin{array}{c}
v_{1}(t) \\
\vdots \\
v_{N}(t)
\end{array}\right)
$$

and the matrices $\mathbb{M}, \mathbb{K}$, and $\mathbb{Q}$, all in $\mathbb{R}^{N_{\text {total }} \times N_{\text {total }} \text {, are }}$

$$
\mathbb{M}_{i, j}=\int_{\Omega} \phi_{i} \phi_{j}, \quad \mathbb{K}_{i, j}=\int_{\Omega} \nabla \phi_{i} \nabla \phi_{j}, \quad \mathbb{Q}_{i, j}=\int_{\partial \Omega} \phi_{i} \phi_{j}, \quad i, j=1, \ldots, N_{\text {total }} .
$$

Finally, the rectangular matrix $\mathbb{B} \in \mathbb{R}^{N \times N_{\text {total }}}$ is defined by

$$
\mathbb{B}=\left[\mathbb{I}_{N \times N} \mid \mathbb{O}_{N \times\left(N_{\text {total }}-N\right)}\right]
$$

where $\mathbb{I}_{N \times N}$ is the identity matrix and $\mathbb{O}_{N \times\left(N_{\text {total }}-N\right)}$ is the appropriate matrix of zeros.

Simple finite differences are used to approximate the reaction-diffusion process on the boundary. Using the same notation, (1.4) is approximated by

$$
\dot{\boldsymbol{u}}=d_{u} \mathbb{D}_{2} \boldsymbol{u}-K_{u}(\boldsymbol{u}-\mathbb{B} \boldsymbol{U})+\sum_{i=1}^{N} f\left(u_{i}, v_{i}\right) e_{i}, \quad \dot{\boldsymbol{v}}=d_{v} \mathbb{D}_{2} \boldsymbol{v}-K_{v}(\boldsymbol{v}-\mathbb{B} \boldsymbol{V})+\sum_{i=1}^{N} g\left(u_{i}, v_{i}\right) e_{i},
$$

where the vectors $e_{i}$ for $i=1, \ldots, N$ form the standard Euclidean basis in $\mathbb{R}^{N}$. Also, $\mathbb{D}_{2} \in \mathbb{R}^{N \times N}$ is the discrete one-dimensional Laplacian with periodic boundary conditions, defined as

$$
\mathbb{D}_{2}=\frac{1}{(d \sigma)^{2}}\left(\begin{array}{ccccc}
-2 & 1 & 0 & \ldots & 1 \\
1 & -2 & 1 & \ldots & 0 \\
\vdots & \ddots & \ddots & \ddots & \vdots \\
0 & \ldots & 1 & -2 & 1 \\
1 & \ldots & 0 & 1 & -2
\end{array}\right)
$$

Now, let $\boldsymbol{W}(t) \in \mathbb{R}^{2 N_{\text {total }}+2 N}$ be the time-dependent solution of the spatially discretized bulk-surface system, defined as

$$
\boldsymbol{W}=\left(\begin{array}{c}
U \\
V \\
u \\
v
\end{array}\right)
$$

Combining the equations in (A.14) with (A.18), we obtain an implicit system of differential equations for $\dot{\boldsymbol{W}}$, given by

$$
\mathbb{C} \dot{\boldsymbol{W}}=\mathbb{A} \boldsymbol{W}+\boldsymbol{F}(\boldsymbol{W})
$$

where $\mathbb{C}, \mathbb{A} \in \mathbb{R}^{\left(2 N_{\text {total }}+2 N\right) \times\left(2 N_{\text {total }}+2 N\right)}$ are block diagonal matrices defined by

$$
\begin{aligned}
& \mathbb{C}=\left(\begin{array}{cccc}
\mathbb{M} & \mathbb{O} & \mathbb{O} & \mathbb{O} \\
\mathbb{O} & \mathbb{M} & \mathbb{O} & \mathbb{O} \\
\mathbb{O} & \mathbb{O} & \mathbb{I}_{N \times N} & \mathbb{O} \\
\mathbb{O} & \mathbb{O} & \mathbb{O} & \mathbb{I}_{N \times N}
\end{array}\right) \\
& \mathbb{A}=\left(\begin{array}{cccc}
-\left(K_{u} \mathbb{Q}+D_{u} \mathbb{K}+\sigma_{u} \mathbb{M}\right) & \mathbb{O} & \mathbb{O} & \mathbb{O} \\
\mathbb{O} & -\left(K_{v} \mathbb{Q}+D_{v} \mathbb{K}+\sigma_{v} \mathbb{M}\right) & \mathbb{O} & \mathbb{O} \\
\mathbb{O} & \mathbb{O} & d_{u} \mathbb{D}_{2} & \mathbb{O} \\
\mathbb{O} & \mathbb{O} & \mathbb{O} & d_{u} \mathbb{D}_{2}
\end{array}\right)
\end{aligned}
$$


where once again each instance of $\mathbb{O}$ is an appropriate matrix of zeros. Finally, the nonlinear terms in (A.21) are defined as

$$
\boldsymbol{F}(\boldsymbol{W})=\left(\begin{array}{c}
K_{u} \mathbb{Q B}^{T} \boldsymbol{u} \\
K_{v} \mathbb{Q B}^{T} \boldsymbol{v} \\
-K_{u}(\boldsymbol{u}-\mathbb{B} \boldsymbol{U})+\sum_{i=1}^{N} f\left(u_{i}, v_{i}\right) e_{i} \\
-K_{v}(\boldsymbol{v}-\mathbb{B} \boldsymbol{V})+\sum_{i=1}^{N} g\left(u_{i}, v_{i}\right) e_{i}
\end{array}\right) .
$$

We conclude this appendix with the definition of two different solution measures used in this paper. First, we define the infinity norm as

$$
\|\boldsymbol{W}\|_{\infty}=\max _{i=1, \ldots, N_{\text {total }}}\left|e_{i}^{T} \boldsymbol{W}\right|
$$

where the set $\left\{e_{i}\right\}$ forms the standard Euclidean basis in $\mathbb{R}^{2 N_{\text {total }}+2 N}$. Then, we can approximate the $L^{2}(\mathcal{W})$ norm using quadratures as

$$
\|\boldsymbol{W}\|_{L^{2}(\mathcal{W})}=\sqrt{\boldsymbol{W}^{T} \tilde{\mathbb{C}} \boldsymbol{W}}
$$

where $\tilde{\mathbb{C}}$ is a mass matrix, defined in terms of $\mathbb{M}($ see $(\mathrm{A} .16))$, by

$$
\tilde{C}=\left(\begin{array}{cccc}
\mathbb{M} & \mathbb{O} & \mathbb{O} & \mathbb{O} \\
\mathbb{O} & \mathbb{M} & \mathbb{O} & \mathbb{O} \\
\mathbb{O} & \mathbb{O} & \mathbb{M}_{N \times N} & \mathbb{O} \\
\mathbb{O} & \mathbb{O} & \mathbb{O} & \mathbb{M}_{N \times N}
\end{array}\right), \quad \text { with } \quad \mathbb{M}_{N \times N}=\frac{d \sigma}{6}\left(\begin{array}{ccccc}
4 & 1 & 0 & \ldots & 1 \\
1 & 4 & 1 & \ldots & 0 \\
\vdots & \ddots & \ddots & \ddots & \vdots \\
0 & \ldots & 1 & 4 & 1 \\
1 & \ldots & 0 & 1 & 4
\end{array}\right) .
$$

\section{A.3 Implicit-explicit time-stepping}

Two different implicit-explicit time-stepping schemes have been used in our PDE numerical simulations: 1-SBDF and 2-SBDF, where the acronym SBDF stands for Semi-Implicit Backward Difference Formula [24]. The single-step method 1-SBDF is employed to obtain the appropriate initial condition for the multi-step method 2-SBDF. When applied to the system (A.21), the two methods yield

$$
\begin{aligned}
& 1-\mathrm{SBDF}:(\mathbb{C}-\Delta t \mathbb{A}) \boldsymbol{W}^{n+1}=\mathbb{C} \boldsymbol{W}^{n}+\Delta t \boldsymbol{F}\left(\boldsymbol{W}^{n}\right), \\
& 2-\mathrm{SBDF}:(3 \mathbb{C}-2 \Delta t \mathbb{A}) \boldsymbol{W}^{n+1}=4 \mathbb{C} \boldsymbol{W}^{n}+4 \Delta t \boldsymbol{F}\left(\boldsymbol{W}^{n}\right)-\mathbb{C} \boldsymbol{W}^{n-1}-2 \Delta t \boldsymbol{F}\left(\boldsymbol{W}^{n-1}\right),
\end{aligned}
$$

where $\Delta t$ is the time step and $\boldsymbol{W}^{n} \approx \boldsymbol{W}(n \Delta t)$ is the approximate solution. Hence, given an initial condition $\boldsymbol{W}^{0}$, we can compute the solution at the next time step $\boldsymbol{W}^{1}$ using 1-SBDF, after which both $\boldsymbol{W}^{0}$ and $\boldsymbol{W}^{1}$ are used as initial conditions in 2-SBDF.

The same time-stepping can also be applied to the spatially discretized radially symmetric system (A.3), where one simply needs to replace the matrix $\mathbb{C}$ with the appropriate identity matrix.

Finally, we remark that the time step $\Delta t$ used in our simulations never exceeded $10^{-2}$.

\section{Appendix B Nondimensionalization}

We derive here the dimensionless coupled bulk-surface reaction-diffusion system defined in equations (1.2)-(1.4). Let us first consider an arbitrary two-dimensional bounded bulk domain $\Omega_{\xi}$, along with its one-dimensional boundary $\partial \Omega_{\xi}$. If $\mathcal{U}(\xi, T)$ and $\mathcal{V}(\xi, T)$ denote the bulk variables undergoing diffusion and linear decay, then they must satisfy

$$
\frac{\partial \mathcal{U}}{\partial T}=D_{\mathcal{U}} \Delta \mathcal{U}-\sigma_{\mathcal{U}} \mathcal{U}, \quad \frac{\partial \mathcal{V}}{\partial T}=D_{\mathcal{V}} \Delta \mathcal{V}-\sigma_{\mathcal{V}} \mathcal{V}, \quad \xi \in \Omega_{\xi}, \quad T>0
$$

Here $D_{\mathcal{U}}$ and $D_{\mathcal{V}}$ are bulk diffusion coefficients, while $\sigma_{\mathcal{U}}$ and $\sigma_{\mathcal{V}}$ are constant decay rates. Next, equations (B.1) are supplemented with linear Robin-type boundary conditions given by

$$
D_{\mathcal{U}}\left(\partial_{n_{\xi}} \mathcal{U}\right)=K_{\mathfrak{u}} \mathfrak{u}-K_{\mathcal{U}} \mathcal{U}, \quad D_{\mathcal{V}}\left(\partial_{n_{\xi}} \mathcal{V}\right)=K_{\mathfrak{v}} \mathfrak{v}-K_{\mathcal{V}} \mathcal{V}, \quad \xi \in \partial \Omega_{\xi}
$$

where $n_{\xi}$ is the outward normal unit vector to the domain $\Omega_{\xi}$ while $\mathfrak{u}, \mathfrak{v}$ are membrane-bound variables. The four coupling parameters $K_{\mathfrak{u}}, K_{\mathfrak{v}}, K_{\mathcal{U}}$ and $K_{\mathcal{V}}$ in equation (B.2) model local exchanges between membrane-bound and bulk variables. On the boundary $\partial \Omega_{\xi}$, the dynamics of $\mathfrak{u}(\xi, T)$ and $\mathfrak{v}(\xi, \mathfrak{T})$ are governed by a system of reaction-diffusion equations of the form

$$
\frac{\partial \mathfrak{u}}{\partial T}=d_{\mathfrak{u}} \Delta_{s} \mathfrak{u}-K_{\mathfrak{u}} \mathfrak{u}+K_{\mathcal{U}} \mathcal{U}+\gamma_{\mathfrak{u}} \frac{\mu}{L} f\left(\frac{L \mathfrak{u}}{\mu}, \frac{L \mathfrak{v}}{\mu}\right), \quad \frac{\partial \mathfrak{v}}{\partial T}=d_{\mathfrak{v}} \Delta_{s} \mathfrak{v}-K_{\mathfrak{v}} v+K_{\mathcal{V}} \mathcal{V}+\gamma_{\mathfrak{v}} \frac{\mu}{L} g\left(\frac{L \mathfrak{u}}{\mu}, \frac{L \mathfrak{v}}{\mu}\right), \quad \xi \in \partial \Omega_{\xi},
$$

where $d_{\mathfrak{u}}$ and $d_{\mathfrak{v}}$ are surface diffusion coefficients, while $f(\cdot, \cdot)$ and $g(\cdot, \cdot)$ are arbitrary dimensionless nonlinear reaction kinetics. Here, $\mu, L, \gamma_{\mathfrak{u}}$ and $\gamma_{\mathfrak{v}}$ are respectively typical mass, dimension and time-scale measures, needed for the units to balance in each equation. 
Next, we explicitly state the units of the variables,

$$
[\xi]:[\text { length }], \quad[\mathrm{T}]:[\text { time }], \quad[\mathfrak{u}],[\mathfrak{v}]: \frac{[\text { mass }]}{[\text { length }]}, \quad[\mathcal{U}],[\mathcal{V}]: \frac{[\text { mass }]}{[\text { length }]^{2}},
$$

and the units of the parameters,

$$
\begin{aligned}
& {[L]:[\text { length }], \quad[\mu]:[\text { mass }], \quad \mathrm{K}_{\mathfrak{u}}, \mathrm{K}_{\mathfrak{v}}: \frac{1}{[\text { time }]}, \quad\left[\mathrm{K}_{\mathcal{U}}\right],\left[\mathrm{K}_{\mathcal{V}}\right]: \frac{[\text { length }]}{[\text { time }]}} \\
& {\left[D_{\mathcal{U}}\right],\left[D_{\mathcal{V}}\right],\left[d_{\mathfrak{u}}\right],\left[d_{\mathfrak{v}}\right]: \frac{[\text { length }]^{2}}{[\text { time }]}, \quad\left[\sigma_{\mathcal{U}}\right],\left[\sigma_{\mathcal{V}}\right],\left[\gamma_{\mathfrak{u}}\right],\left[\gamma_{\mathfrak{v}}\right]: \frac{1}{[\text { time }]}}
\end{aligned}
$$

In this way, new dimensionless variables can be defined as

$$
\begin{aligned}
& t=\gamma_{\mathfrak{u}} T, \quad x=\frac{\xi}{L}, \quad U(x, t)=\frac{L^{2}}{\mu} \mathcal{U}\left(L x, \frac{t}{\gamma_{\mathfrak{u}}}\right), \quad V(x, t)=\frac{L^{2}}{\mu} \mathcal{V}\left(L x, \frac{t}{\gamma_{\mathfrak{u}}}\right), \\
& u(x, t)=\frac{L}{\mu} \mathfrak{u}\left(L x, \frac{t}{\gamma_{\mathfrak{u}}}\right), \quad v(x, t)=\frac{L}{\mu} \mathfrak{v}\left(L x, \frac{t}{\gamma_{\mathfrak{u}}}\right) .
\end{aligned}
$$

We remark that the scaled bulk domain, denoted as $\Omega_{x}$, has a typical dimension of $\mathcal{O}(1)$. For the case of a disk, the typical dimension corresponds to the radius.

Upon substituting the variables defined in (B.6) within the system defined by equations (B.1), (B.2) and (B.3), we readily obtain a dimensionless coupled bulk-surface reaction-diffusion system given by

$$
\begin{aligned}
& \frac{\partial U}{\partial t}=D_{u} \Delta U-\sigma_{u} U, \quad \frac{\partial V}{\partial t}=D_{v} \Delta V-\sigma_{v} V, \quad x \in \Omega_{x}, \quad t>0 \\
& D_{u}\left(\partial_{n_{x}} U\right)=r_{d} u-r_{a} U, \quad D_{v}\left(\partial_{n_{x}} V\right)=p_{d} v-p_{a} V, \quad x \in \partial \Omega_{x} \\
& \frac{\partial u}{\partial t}=d_{u} \Delta_{s} u-r_{d} u+r_{a} U+f(u, v), \quad \frac{\partial v}{\partial t}=d_{v} \Delta_{s} v-p_{d} v+p_{a} V+\gamma g(u, v), \quad x \in \partial \Omega_{x},
\end{aligned}
$$

where the new dimensionless parameters are each defined as

$$
\begin{aligned}
D_{u} & \equiv \frac{D_{\mathcal{U}}}{L^{2} \gamma_{\mathfrak{u}}}, \quad D_{v} \equiv \frac{D_{\mathcal{V}}}{L^{2} \gamma_{\mathfrak{u}}}, \quad d_{u} \equiv \frac{d_{\mathfrak{u}}}{L^{2} \gamma_{\mathfrak{u}}}, \quad d_{v} \equiv \frac{d_{\mathfrak{v}}}{L^{2} \gamma_{\mathfrak{u}}}, \quad \sigma_{u} \equiv \frac{\sigma_{\mathcal{U}}}{\gamma_{\mathfrak{u}}}, \quad \sigma_{v} \equiv \frac{\sigma_{\mathcal{V}}}{\gamma_{\mathfrak{u}}} \\
r_{d} & \equiv \frac{K_{\mathfrak{u}}}{\gamma_{\mathfrak{u}}}, \quad r_{a} \equiv \frac{K_{\mathcal{U}}}{L \gamma_{\mathfrak{u}}}, \quad p_{d} \equiv \frac{K_{\mathfrak{v}}}{\gamma_{\mathfrak{u}}}, \quad p_{a} \equiv \frac{K_{\mathcal{V}}}{L \gamma_{\mathfrak{u}}}, \quad \gamma \equiv \frac{\gamma_{\mathfrak{v}}}{\gamma_{\mathfrak{u}}}
\end{aligned}
$$

Our first assumption is that the time-scales of the nonlinear reaction kinetics are the same, which yields $\gamma=1$. Next, to reduce the size of the parameter space, we will assume equal adsorption and desorption rates for each variable. Letting $K_{u}$ and $K_{v}$ be new coupling rate constants, defined as

$$
K_{u} \equiv r_{a}=r_{d}, \quad K_{v} \equiv p_{a}=p_{d}
$$

we obtain the dimensionless coupled bulk-surface reaction-diffusion system given in $\S 1$. In terms of the parameters of (B.1)-(B.3), we are assuming by (B.9) that

$$
K_{\mathfrak{u}}=\frac{K_{\mathcal{U}}}{L}, \quad K_{\mathfrak{v}}=\frac{K_{\mathcal{V}}}{L} .
$$

However in $§ 5$, we relax assumption (B.9) and allow for distinct adsorption and desorption rates in order to explore the formation of rotating waves in a circular bulk domain.

\section{Acknowledgments}

F. Paquin-Lefebvre was partially supported by a NSERC Doctoral Award and a UBC Four-Year Doctoral Fellowship. W. Nagata and M. J. Ward gratefully acknowledge the support of the NSERC Discovery Grant Program. We would also like to thank Daniel Gomez for discussions on implementing the finite-element solver.

\section{References}

[1] S. M. Baer, T. Erneux, and J. Rinzel. The slow passage through a Hopf bifurcation: delay, memory effects, and resonance. SIAM J. Appl. Math., 49(1):55-71, 1989.

[2] A. R. Champneys and B. Sandstede. Numerical computation of coherent structures. In Numerical Continuation Methods for Dynamical Systems, Underst. Complex Syst., pages 331-358. Springer, Dordrecht, 2007.

[3] M. C. Cross and P. C. Hohenberg. Pattern formation outside of equilibrium. Rev. Mod. Phys., 65:851-1112, Jul 1993. 
[4] H. Dankowicz and F. Schilder. Recipes for Continuation, volume 11 of Computational Science E Engineering. Society for Industrial and Applied Mathematics (SIAM), Philadelphia, PA, 2013.

[5] A. Dhooge, W. Govaerts, and Yu. A. Kuznetsov. MATCONT: a MATLAB package for numerical bifurcation analysis of ODEs. ACM Trans. Math. Software, 29(2):141-164, 2003.

[6] E. J. Doedel, A. R. Champneys, T. Fairgrieve, Y. Kuznetsov, B. Oldeman, R. Paffenroth, B. Sandstede, X. Wang, and C. Zhang. Auto07p: Continuation and bifurcation software for ordinary differential equations. Technical report, Concordia University, 2007.

[7] A. Gomez-Marin, J. Garcia-Ojalvo, and J. M. Sancho. Self-sustained spatiotemporal oscillations induced by membrane-bulk coupling. Phys. Rev. Lett., 98:168303, Apr 2007.

[8] J. Gou and M. J. Ward. Oscillatory dynamics for a coupled membrane-bulk diffusion model with Fitzhugh-Nagumo membrane kinetics. SIAM J. Appl. Math., 76(2):776-804, 2016.

[9] J. Gou, Y. X. Li, W. Nagata, and M. J. Ward. Synchronized oscillatory dynamics for a 1-D model of membrane kinetics coupled by linear bulk diffusion. SIAM J. Appl. Dyn. Syst., 14(4):2096-2137, 2015.

[10] J. Gou, W.-Y. Chiang, P.-Y. Lai, M. J. Ward, and Y.-X. Li. A theory of synchrony by coupling through a diffusive chemical signal. Phys. D, 339:1-17, 2017.

[11] Jia Gou, Wayne Nagata, and Yue-Xian Li. Interaction of in-phase and anti-phase synchronies in a coupled compartment-bulk diffusion model at a double Hopf bifurcation. IMA J. Appl. Math., 81(6):1137-1162, 2016.

[12] C. Grossmann, H.G. Roos, and M. Stynes. Numerical Treatment of Partial Differential Equations. Universitext. Springer Berlin Heidelberg, 2007.

[13] J. Guckenheimer and P. Holmes. Nonlinear Oscillations, Dynamical Systems, and Bifurcations of Vector Fields, volume 42 of Applied Mathematical Sciences. Springer-Verlag, New York, 1983.

[14] J. Halatek and E. Frey. Highly canalized mind transfer and mine sequestration explain the origin of robust mincdeprotein dynamics. Cell Reports, 1(6):741-752, 2012.

[15] J. Halatek, F. Brauns, and E. Frey. Self-organization principles of intracellular pattern formation. Phil. Trans. R. Soc. B, 373(1747):20170107, 2018.

[16] K. Korvasová, E.A. Gaffney, P.K. Maini, M.A. Ferreira, and V. Klika. Investigating the Turing conditions for diffusion-driven instability in the presence of a binding immobile substrate. J. Theor. Biol., 367:286 - 295, 2015.

[17] Y. A. Kuznetsov. Elements of Applied Bifurcation Theory, volume 112 of Applied Mathematical Sciences. SpringerVerlag, New York, 2004.

[18] H. Levine and W.-J. Rappel. Membrane-bound Turing patterns. Phys. Rev. E, 72:061912, Dec 2005.

[19] A. Madzvamuse and A. H. W. Chung. The bulk-surface finite element method for reaction-diffusion systems on stationary volumes. Finite Elements in Analysis and Design, 108:9 - 21, 2016.

[20] A. Madzvamuse, A. H. W. Chung, and C. Venkataraman. Stability analysis and simulations of coupled bulk-surface reaction-diffusion systems. Proc. R. Soc., Ser. A, 471(2175):20140546, 18, 2015.

[21] MATLAB. Partial Differential Equations Toolbox (R2016b). The MathWorks Inc., Natick, Massachusetts, 2016.

[22] A. Rätz and M. Röger. Turing instabilities in a mathematical model for signaling networks. J. Math. Biol., 65(6-7): $1215-1244,2012$.

[23] A. Rätz and M. Röger. Symmetry breaking in a bulk-surface reaction-diffusion model for signalling networks. Nonlinearity, 27(8):1805, 2014.

[24] S. J. Ruuth. Implicit-explicit methods for reaction-diffusion problems in pattern formation. J. Math. Biol., 34(2): 148-176, 1995.

[25] H. Uecker, D. Wetzel, and J. D. M. Rademacher. pde2path-a Matlab package for continuation and bifurcation in 2D elliptic systems. Numer. Math. Theory Methods Appl., 7(1):58-106, 2014.

[26] D. Walgraef. Spatio-temporal Pattern Formation, volume 2 of Partially Ordered Systems. Springer-Verlag, New York, 1997.

[27] S. Wiggins. Introduction to Applied Nonlinear Dynamical Systems and Chaos, volume 2 of Texts in Applied Mathematics. Springer-Verlag, New York, 2003.

[28] R. W. Wittenberg and P. Holmes. The limited effectiveness of normal forms: a critical review and extension of local bifurcation studies of the Brusselator PDE. Phys. D, 100(1-2):1-40, 1997. 